\title{
Differential Equations in the Spectral Parameter *
}

\author{
J. J. Duistermaat ${ }^{1}$ and F. A. Grünbaum ${ }^{2}$ \\ 1 Mathematisch Instituut der Rijksuniversiteit Utrecht, The Netherlands \\ 2 Department of Mathematics, University of California, Berkeley, CA94720, USA
}

\begin{abstract}
We determine all the potentials $V(x)$ for the Schrödinger equation $\left(-\partial_{x}^{2}+\mathrm{V}(x)\right) \phi=\mathrm{k}^{2} \phi$ such that some family of eigenfunctions $\phi$ satisfies a differential equation in the spectral parameter $k$ of the form $B\left(k, \partial_{k}\right) \phi=\Theta(x) \phi$. For each such $V(x)$ we determine the algebra of all possible operators $B$ and the corresponding functions $\Theta(x)$.
\end{abstract}

\section{Table of Contents}

0. Introduction . . . . . . . . . . . . . . . . . . . . . . . 177

1. $(\operatorname{ad} L)^{m+1}(\Theta)=0$. . . . . . . . . . . . . . . . . . . . . . . . . . 180

2. $V(\infty)$ is Finite . . . . . . . . . . . . . . . . . . . . . . . . . . 187

3. The Rational KdV Potentials . . . . . . . . . . . . . . . . . . . . . 192

4. The Even Case . . . . . . . . . . . . . . . . . . . . . . . . . . 203

5. The Even Potentials Work Too . . . . . . . . . . . . . . . . . . . . 213

6. $V(\infty)=\infty$ is the Airy Case. . . . . . . . . . . . . . . . . . . . . . 218

7. Some Illustrative Examples . . . . . . . . . . . . . . . . . . . . . 222

\section{Introduction}

In this paper we study the following question: For which linear ordinary differential operators $L=\sum_{j=0}^{l} L_{j}(x) \cdot\left(\frac{\partial}{\partial x}\right)^{j}$ is there a non-zero family of eigenfunc-
tions $\phi(x, \lambda)$,

$$
(L \phi)(x, \lambda)=\lambda \cdot \phi(x, \lambda),
$$

depending smoothly on the eigenfunction parameter $\lambda$, which is also an eigenfunction of a linear ordinary differential operator $A=\sum_{r=0}^{m} A_{r}(\lambda) \cdot\left(\frac{\partial}{\partial \lambda}\right)^{r}$

$$
(A \phi)(x, \lambda)=\Theta(x) \cdot \phi(x, \lambda)
$$

for an eigenvalue $\Theta$ which is a function of $x$ ?

* This research was partially supported by NSF grant DMS 84-03232 and ONR contract NOOO 14-84-C-0159 
One of us was led to this question in an attempt to analyze some problems in "limited angle tomography," see [11].

The quantitative study of the relation between "amount of data" and "picture quality" rests on the possibility of a detailed analysis of the spectral properties of a specific integral operator. This is made possible, in very simple cases, by the miraculous existence of a commuting second-order differential operator: the same one that enters in the study of the problem of concentrating a function both in time and frequency, see Slepian and its references [24], or in the study of the eigenvalue distribution for infinite matrices with Gaussian entries, see Mehta [18].

Both for practical as well as for purely mathematical reasons it is desirable to look at the corresponding integral operator in more complicated situations than the real line, or equivalently in the case when Fourier analysis is replaced by the decomposition in terms of eigenfunctions of a general second order differential operator on the line. In $[12,13]$ several cases are considered in detail, and the observation is made that there is a relation between the existence of a commuting differential operator and the validity of property $(0.2)$. How strict this relation is, remains an open problem.

Another motivation was that one frequently sees families of eigenfunctions $\phi(x, \lambda)$ for which the asymptotics as $\lambda \rightarrow \infty$ has a formal resemblance to the asymptotics for $x \rightarrow \infty$. The asymptotics for $x \rightarrow \infty$ follows from the differential equation (0.1) in the variable $x$. A differential equation (0.2) in the variable $\lambda$ could then be the explanation of the similarity of the asymptotics.

We will give a detailed answer to the case that $L$ has order 2 , when it can be taken in the standard Schrödinger form

$$
L=-\left(\frac{\partial}{\partial x}\right)^{2}+V(x)
$$

with some potential $V(x)$. The answer will be described in terms of a construction introduced in 1882 by Darboux [6, Livre IV, Chap. IX, No. 408], and which we now recall for the convenience of the reader.

One can always factorize

$$
L=P \circ Q \quad \text { with } \quad P=-\frac{\partial}{\partial x}-\frac{\phi_{0}^{\prime}(x)}{\phi_{0}(x)}, \quad Q=\frac{\partial}{\partial x}-\frac{\phi_{0}^{\prime}(x)}{\phi_{0}(x)},
$$

where $\phi_{0}$ is a non-zero eigenfunction for the eigenvalue 0 :

$$
L \phi_{0}=0 \text {. }
$$

Interchanging the factors one obtains another Schrödinger operator

$$
\tilde{L}=Q \circ P=-\left(\frac{\partial}{\partial x}\right)^{2}+\tilde{V}(x)
$$

with

$$
\tilde{V}(x)=V(x)-2\left(\frac{\phi_{0}^{\prime}(x)}{\phi_{0}(x)}\right)^{\prime}
$$

as the new potential. The point is that if $L \phi=\lambda \phi$, then $\tilde{L} Q \phi=Q P Q \phi=Q L \phi=\lambda Q \phi$, so if one knows the eigenfunctions for $L$, then one obtains the eigenfunctions for $\widetilde{L}$ 
by applying $Q$ to them. (This explanation, in terms of interchanging the factors, is due to Burchnall and Chaundy [5], who call it "transference.") We shall call $V \mapsto \tilde{V}$ a rational Darboux transformation if $\phi_{0}^{\prime}(x) / \phi_{0}(x)$ is rational. With such a transformation, $\tilde{V}$ remains rational if $V$ is rational. Our answer to the question posed above can now be formulated as follows.

Theorem 0.1. The potentials $V$ for which (0.1), (0.2) hold ( for non-zero $\phi$ and $A$ of positive order) are $V(x)=\alpha x+\beta, \alpha, \beta \in \mathbb{C}, \alpha \neq 0$ (Airy) or $V(x)=\frac{c}{(x-a)^{2}}+b$, $a, b, c \in \mathbb{C}$ (Bessel) or, modulo a translation in $x$ and adding a constant to $V$, those which can be obtained from $V=0$ or $V=-\frac{1}{4} \frac{1}{x^{2}}$ by finitely many rational Darboux
transformations.

With the Darboux transformations starting from $V=0$, one can, at every step, take an arbitrary non-zero $\phi_{0} \in \operatorname{Ker} L$. Because a non-zero factor in $\phi_{0}$ leads to the same new potential, at every step this introduces one new complex parameter in the family of potentials; this parameter runs over the Riemann sphere of complex lines in the complex two-dimensional vector space $\operatorname{Ker} L$. The potentials thus obtained have been identified by Adler and Moser [2] as the potentials which stay rational under the Korteweg-de Vries flow, as found in the paper of Airault, McKean, Moser [3]. An equivalent characterization of these potentials is given by the condition that $V(\infty)$ is finite and that all the eigenfunctions are meromorphic in $\mathbb{C}$, see Theorem 3.4.

For the Darboux transformations starting at $-\frac{1}{4} x^{2}$, the rationality of $\phi_{0}^{\prime} / \phi_{0}$ at the various steps is no longer automatic; see Proposition 4.3 and the remarks thereafter for more details. This case is characterized by the property that, after conjugating $A$ by a suitable function of $\lambda$, the common eigenspace $\operatorname{Ker}(L-\lambda)$ $\cap \operatorname{Ker}(A-\Theta(x))$ is 2-dimensional, whereas it is always 1-dimensional for the rational $\mathrm{KdV}$ potentials which are not Bessel. Moreover, if $\operatorname{dim}[\operatorname{Ker}(L-\lambda)$ $\cap \operatorname{Ker}(A-\Theta(x))]=2$, then $V$ and $\Theta$ are necessarily even functions of $x$, which is why we call this the "even case." A natural question would be to find evolution equations analogous to the $\mathrm{KdV}$ hierarchy in the even case.

Equivalent descriptions of the rational $\mathrm{KdV}$ potentials, respectively the potentials in the even case, can be given in terms of the properties of their poles in the complex plane, see (3.31). (3.32), respectively (4.45), (4.46). The operators appearing in (0.2) form an algebra (corresponding to a singular curve) isomorphic to the algebra of functions $\Theta$ in (0.2). These turn out to be polynomials, characterized by the vanishing of the first $v_{p}$ derivatives of odd order of $\Theta$ at the poles $p(p \neq 0$ in the even case) of the potential $V$ (see Theorems 3.5 and 5.4). A fringe benefit is the relative ease with which we can produce examples of such algebras of differential operators.

The first step in our proofs is the observation that $\Theta$ must be a polynomial and $V$ must be rational. The argument is related to, again, a basic observation in Burchnall and Chaundy [5]. Then all other results are obtained from an analysis of the asymptotic behavior of the eigenfunctions $\phi(x, \lambda)$ as $x$ converges to $\infty$, or to a pole of $V$, or as $\lambda \rightarrow \infty$. In this we follow the strategy of Flaschka and Newell [8]. For instance, the somewhat mysterious equation (3.32), (4.46) simply express that 
the eigenfunctions are single-valued around these poles $p$ of $V$ for all $\lambda$; see Proposition 3.3. In Sect. 7 we discuss some special cases in more detail, in order to illustrate some aspects which have received only marginal attention in the general theory.

After unearthing the final answer (in the process of which we got our hands very dirty), we are still left with feelings of bewilderment. For instance, the rational KdV potentials, respectively, the ones in the even case, bifurcate from the Bessel potentials $\frac{c}{x^{2}}$ at $c=v(v+1)$, respectively $c=L^{2}-\frac{1}{4}=\left(L-\frac{1}{2}\right)\left(L+\frac{1}{2}\right)$ with $v, L \in \mathbb{Z}$. Reading $x=\left(x_{1}^{2}+\ldots+x_{n}^{2}\right)^{1 / 2}$, the eigenfunctions at these "bifurcation Bessel potentials" are, up to a factor $x^{-\frac{n-1}{2}}$, the spherical symmetric eigenfunctions for the Laplace operator in $\mathbb{R}^{n}$, with $n=2 v+3$, respectively, $n=2 L+2$. Does this mean that there is a geometric explanation for our answer?

\section{1. $(\operatorname{ad} L)^{m+1}(\Theta)=0$}

Consider a linear ordinary differential operator

$$
L=L\left(x, \partial_{x}\right)=\sum_{j=0}^{l} L_{j}(x) \partial_{x}^{j}, \quad \partial_{x}=\frac{\partial}{\partial x},
$$

of order $l$, with complex valued coefficients $L_{j}(x)$, depending smoothly on the independent variable $x$. Let $\phi=\phi(x, \lambda)$ be a non-zero family of eigenfunctions of $L$,

$$
(L \phi)(x, \lambda)=\lambda \cdot \phi(x, \lambda),
$$

depending smoothly on $x$ and $\lambda$, which satisfies another differential equation of the form

$$
(A \phi)(x, \lambda)=(\Theta \phi)(x, \lambda) .
$$

Here $A$ is a linear differential operator with respect to $\lambda$,

$$
A=A\left(\lambda, \partial_{\lambda}\right)=\sum_{r=0}^{m} A_{r}(\lambda) \partial_{\lambda}^{r}, \quad \partial_{\lambda}=\frac{\partial}{\partial \lambda}
$$

with coefficients depending only on $\lambda$, and

$$
\Theta=\Theta\left(x, \partial_{x}\right)=\sum_{s=0}^{m} \Theta_{s}(x) \partial_{x}^{s},
$$

is a linear differential operator with respect to $x$, with coefficients depending (smoothly) on $x$.

The assumptions are made only locally, that is, for $x$, respectively $\lambda$, in a neighborhood of $x_{0}$, respectively $\lambda_{0}$, on the real axis. "Smooth" means $C^{p}$ for sufficiently large $p . p=l(m+1)$ is safe, it is a separate game to find minimal smoothness conditions for $\phi$ and the coefficients of $L$ and $\Theta$ in order that the conclusions in this section remain valid.

Write

$$
(\operatorname{ad} P)(Q)=[P, Q]=P Q-Q P,
$$


for the commutator of two linear operators $P, Q$. One obtains immediately from (1.2) and (1.3) that

$$
[L, \Theta] \phi=[-\lambda, A] \phi,
$$

using that $A=A\left(\lambda, \partial_{\lambda}\right)$ and $L=L\left(x, \partial_{x}\right)$, respectively $\lambda$ and $\Theta=\Theta\left(x, \partial_{x}\right)$, commute with each other. Replacing $\Theta$, respectively $A$, in $(1.7)$ by $(\operatorname{ad} L)^{r-1}(\Theta)$, respectively $(-\operatorname{ad} \lambda)^{r-1}(A)$, one obtains by induction on $r$ that

$$
(\operatorname{ad} L)^{r}(\Theta) \phi=(-\operatorname{ad} \lambda)^{r}(A) \phi \text { for all } r \in \mathbb{Z}>0 .
$$

Here the zero order differential operator "multiplication by $\lambda$ " has been denoted by $\lambda$ as well. Because each ad $\lambda$ decreases the order of a linear differential operator in $\lambda$, we get from (1.8) with $r=m+1$ :

$$
(\operatorname{ad} L)^{m+1}(\Theta) \phi=0 .
$$

On the other hand, ad $L$ increases the order of a linear differential operator in $x$ by at most $l-1$, so $(\operatorname{ad} L)^{m+1}(\Theta)$ is a linear differential operator in $x$, with coefficients depending only on $x$, of order at most $a=\operatorname{order} \Theta+(m+1)(l-1)$.

Now let $(\operatorname{ad} L)^{m+1}(\Theta) \neq 0$, order $(\operatorname{ad} L)^{m+1}(\Theta)=\tilde{a} \leqq a$. In a neighborhood of a point $\tilde{x}_{0}$ where the coefficient of the highest order term does not vanish, the null space of $(\operatorname{ad} L)^{m+1}(\Theta)$ is a vector space of functions of $x$ of finite dimension, equal to $\tilde{a}$. However, non-zero eigenvectors for any linear operator $L$, corresponding to different eigenvalues $\lambda_{1}, \ldots, \lambda_{t}$, are automatically linearly independent. So taking $t>\tilde{a}, \phi=\phi\left(\cdot, \lambda_{s}\right), s=1, \ldots, t$ in (1.9), we arrive at a contradiction with the assumption that $(\operatorname{ad} L)^{m+1}(\Theta) \neq 0$. (This is the argument with which Burchnall and Chaundy [5] proved that two commuting ordinary differential operators satisfy an algebraic equation between them.) We have proved that necessarily

$$
(\operatorname{ad} L)^{m+1}(\Theta)=0 \text {. }
$$

Equation (1.10) can be given another, equivalent formulation, using the following infinitesimal version of the linear independence of the eigenfunctions $\phi(\cdot, \lambda)$ for different $\lambda$ 's.

Lemma 1.1. Let $\left(\phi_{j}(\cdot, \lambda)\right)_{j=1, \ldots, l}$ be a basis of $\operatorname{Ker}(L-\lambda I)$, each $\phi_{j}(\cdot, \lambda)$ depending smoothly on $\lambda$. Then

$$
\left(\partial_{\lambda}^{p} \phi_{j}\right)(\cdot, \lambda)_{j=1, \ldots, l}^{p=0, \ldots, m}
$$

forms a basis of $\operatorname{Ker}(L-\lambda I)^{m+1}$.

Proof. For any $\phi(x, \lambda)$ depending smoothly on $x, \lambda$ :

$$
(L-\lambda I) \partial_{\lambda}^{p} \phi=p \cdot \partial_{\lambda}^{p-1} \phi .
$$

The proof is by induction on $p$, the induction step is made by differentiating (1.12) with respect to $\lambda$. Applying powers of $L-\lambda I$ to (1.12) yields

$$
\begin{aligned}
& (L-\lambda I)^{q} \cdot \partial_{\lambda}^{p} \phi=\frac{p !}{(p-q) !} \partial_{\lambda}^{p-q} \phi \text { for } q \leqq p, \\
& (L-\lambda I)^{p+1} \cdot \partial_{\lambda}^{p} \phi=0 \text { if } \phi \in \operatorname{Ker}(L-\lambda I) .
\end{aligned}
$$


So the elements in (1.11) all belong to $\operatorname{Ker}(L-\lambda I)^{m+1}$. Suppose now that $c_{j p}$ are constants such that

$$
\sum_{j=1}^{l} \sum_{p=0}^{m} c_{j p} \cdot \partial_{\lambda}^{p} \phi_{j}=0 .
$$

Applying $(L-\lambda I)^{m}$ we get, using (1.13), (1.14) that

$$
\sum_{j=1}^{l} c_{j m} \cdot \phi_{j}=0,
$$

which, in view of the linear independence of the $\phi_{j}$, means that $c_{j m}=0$ for all $j$. By induction on $m$ it follows that $c_{j p}=0$ for all $j, p$ and we have proved that the $\partial_{\lambda}^{p} \phi_{j}$ are linearly independent. Because

$$
\operatorname{dim} \operatorname{Ker}(L-\lambda I)^{m+1}=\operatorname{order}(L-\lambda I)^{m+1}=(m+1) l,
$$

the $\partial_{\lambda}^{p} \phi_{j}$ form a basis of $\operatorname{Ker}(L-\lambda I)^{m+1}$.

Proposition 1.2. The following conditions a)-c) are equivalent:

a) There is a non-zero family $\phi(\cdot, \lambda) \in \operatorname{Ker}(L-\lambda I)$, depending smoothly on $\lambda$, such that $(\Theta \phi)(\cdot, \lambda) \in \operatorname{Ker}(L-\lambda I)^{m+1}$ for all $\lambda$.

b) $(\operatorname{ad} L)^{m+1}(\Theta)=0$.

c) $\Theta$ maps $\operatorname{Ker}(L-\lambda I)$ into $\operatorname{Ker}(L-\lambda I)^{m+1}$ for all $\lambda$.

Proof. If $\phi \in \operatorname{Ker}(L-\lambda I)$ then, by induction on $m$ :

$$
(L-\lambda I)^{m+1}(\Theta) \cdot \phi=\left((\operatorname{ad} L)^{m+1}(\Theta)\right) \phi .
$$

So, if $\phi$ is as in a), then $\left((\operatorname{ad} L)^{m+1}(\Theta)\right) \phi(\cdot, \lambda)=0$ for all $\lambda$. But $(1.9) \Rightarrow(1.10)$ above then gives $b)$. b) $\Rightarrow$ c) follows directly from $(1.15)$ and $c) \Rightarrow$ a) is obvious.

We will apply Proposition 1.2 and Lemma 1.1 in the next sections in the following way. Let $\mathscr{2}$ be a linear subspace of the space of functions of $x$, characterized for instance by certain asymptotic behavior, such that

$$
\operatorname{dim}\left(\mathscr{Q} \cap \operatorname{Ker}(L-\lambda I)^{m+1}\right) \leqq m+1 .
$$

Let $\hat{\phi}(\cdot, \lambda)$ be a non-zero family of functions of $x$, depending smoothly on $\lambda$, such that

$\hat{\phi}(\cdot, \lambda) \in \operatorname{Ker}(L-\lambda I) \cap \mathscr{Q}, \quad(\Theta \hat{\phi})(\cdot, \lambda) \in \mathscr{Q} \quad$ and $\quad \partial_{\lambda}^{p} \hat{\phi}(\cdot, \lambda) \in \mathscr{Q} \quad$ for all $\quad 0 \leqq p \leqq m$.

Inequality (1.16) and the last statement in (1.17) imply, in view of Lemma 1.1, that the $\partial_{\lambda}^{p} \hat{\phi}(\cdot, \lambda), 0 \leqq p \leqq m$, form a basis of $\mathscr{Q} \cap \operatorname{Ker}(L-\lambda I)^{m+1}$ [and the equality holds in (1.16)]. From Proposition 1.2 we now read off:

Lemma 1.3. If $(\operatorname{ad} L)^{m+1}(\Theta)=0$ and $(1.16),(1.17)$ hold, then $(\Theta \hat{\phi})(\cdot, \lambda)$ is a linear combination of the $\partial_{\lambda}^{p} \hat{\phi}(\cdot, \lambda), 0 \leqq p \leqq m$, with coefficients depending only on $\lambda$. That is, $\hat{\phi}$ satisfies an equation of the form (1.3), replacing $A$ by a suitable operator $\hat{A}=\hat{A}\left(\lambda, \partial_{\lambda}\right)$.

This leads to equivalence between the equation $(\operatorname{ad} L)^{m+1}(\Theta)=0$ and the existence of a non-zero family $\phi=\phi(x, \lambda)$ and an operator $A=A\left(\lambda, \partial_{\lambda}\right)$ satisfying (1.2), (1.3). See Sects. 2 and 6 , where the subspace $\mathscr{Q}$ is defined by the asymptotic 
behaviour for $x \rightarrow \infty$. From the existence of a non-zero family $\phi=\phi(x, \lambda)$ and operator $A=A\left(\lambda, \partial_{\lambda}\right)$ satisfying (1.2), (1.3) we will use Lemma 1.3 again in Lemma 2.1 in order to see that the special family $\hat{\phi}=\hat{\phi}(\cdot, \lambda) \in \mathscr{Q}$ satisfies (1.2), (1.3) as well, with $A$ replaced by a suitable operator $\hat{A}=\hat{A}\left(\lambda, \partial_{\lambda}\right)$.

From now on let us assume that

$$
\Theta=\Theta(x)
$$

is of zero order - so it plays the role of an eigenvalue parameter for the operator $A=A\left(\lambda, \partial_{\lambda}\right)$ in (1.3). (One has to allow $\Theta$ to depend on $x$ in order to get a non-trivial problem.)

Assuming also that $L_{l}(x)$, the highest order coefficient of $L$ is real, or that $L$ has analytic coefficients and therefore is defined in a complex neighborhood of $x_{0}$, we can arrange by a substitution of variables in $x$ that

$$
L_{l}(x) \text { is a non-zero constant. }
$$

The coefficient of $\partial_{x}^{(m+1)(l-1)}$ in $(\operatorname{ad} L)^{m+1}(\Theta)$ now is equal to $\left(-l \cdot L_{l}\right)^{m+1} \cdot \partial_{x}^{m+1} \Theta$, so $(\operatorname{ad} L)^{m+1}(\Theta)=0$ implies that

$$
\Theta(x) \text { is a polynomial in } x \text { of degree } \leqq m .
$$

Conjugating $L$ with a suitable function of $x$ we can also arrange that

$$
L_{l-1}(x) \equiv 0 \quad \text { (Liouville) }
$$

Restricting ourselves to second order operators $L$, as we will from now on, we may therefore take it in the conventional (Schrödinger) form:

$$
L\left(x, \partial_{x}\right)=-\partial_{x}^{2}+V(x)
$$

where the zero order term $V(x)$ is called the potential. Writing

$$
\left(\operatorname{ad}\left(\partial_{x}^{2}-V\right)\right)^{m+1}(\Theta)=\sum_{\substack{l_{1}, \ldots, l_{p} \\ \Sigma l_{j}=m+1}} \ldots \circ\left(\operatorname{ad} \partial_{x}^{2}\right)^{l_{3}} \circ(-\operatorname{ad} V)^{l_{2}} \circ\left(\operatorname{ad} \partial_{x}^{2}\right)^{l_{1}}(\Theta),
$$

the part which is homogeneous in $V$ of degree $d$ is equal to the sum on the righthand side over all $l_{1}, \ldots, l_{p}$ such that $\sum l_{\text {even }}=d$. Because ad $V$ decreases the order of a linear differential operator in $x$ and ad $\partial_{x}^{2}$ increases it by at most one, this part has order at most equal to $\sum l_{\text {odd }}-\sum l_{\text {even }}=\sum_{j} l_{j}-2 \sum l_{\text {even }}=m+1-2 d$. In particular, we see that the coefficient of $\partial_{x}^{m}$ in $(\operatorname{ad} L)^{m+1}(\Theta)$ does not involve $V$ yet.

Using that

$$
\left[\partial_{x}^{2}, c(x)\right]=2 c^{\prime}(x) \partial_{x}+c^{\prime \prime}(x)
$$

it follows by induction over $j$ that

$$
\left(\operatorname{ad} \partial_{x}^{2}\right)^{j}\left(\sum_{q=0}^{p} c_{q}(x) \partial_{x}^{q}\right)=\sum_{r=0}^{p+j} \sum_{\substack{s=0 \\
s \geqq j-r}}^{j} 2^{j-s} \cdot\left(\begin{array}{l}
j \\
s
\end{array}\right) \cdot c_{r-j+s}^{(j+s)} \cdot \partial_{x}^{r} .
$$

The part of $(-\operatorname{ad} L)^{m+1}(\Theta)$ which does not involve $V$ is obtained by putting $j=m+1, p=0, c_{0}=\Theta, s=j-r=m+1-r$, hence $j+s=2(m+1)-r \geqq m+1$ in (1.25), and we conclude that it vanishes if $(1.20)$ holds. In particular, $(\operatorname{ad} L)^{m+1}(\Theta)$ is already of order $\leqq m-1$ if $(1.20)$ holds. 
The coefficient of $\partial_{x}^{m-1}$ in $(-\operatorname{ad} L)^{m+1}(\Theta)$ is now linear in $V$, and equal to the highest order coefficient in

$$
\sum_{j=1}^{m}\left(\operatorname{ad} \partial_{x}^{2}\right)^{m-j} \circ(-\operatorname{ad} V) \circ\left(\operatorname{ad} \partial_{x}^{2}\right)^{j}(\Theta)
$$

Now using that

$$
(-\operatorname{ad} V) \partial_{x}^{q}=\sum_{r=1}^{q}\left(\begin{array}{l}
q \\
r
\end{array}\right) \cdot V^{(r)} \cdot \partial_{x}^{q-r}=q \cdot V^{\prime} \cdot \partial_{x}^{q-1}+\begin{array}{r}
\text { lower } \\
\text { order } \\
\text { terms }
\end{array}
$$

the coefficient of $\partial_{x}^{m-1}$ in $(-\operatorname{ad} L)^{m+1}(\Theta)$ comes out as

$$
\begin{aligned}
\sum_{j=1}^{m} & 2^{m-j} \cdot\left(j \cdot V^{\prime} \cdot 2^{j} \cdot \Theta^{(j)}\right)^{(m-j)} \\
& =2^{m} \cdot \sum_{j=1}^{m} j \cdot \sum_{l=0}^{m-j}\left(\begin{array}{c}
m-j \\
l
\end{array}\right) \cdot V^{(l+1)} \cdot \Theta^{(m-l)} \\
& =2^{m} \sum_{l=0}^{m-1}\left(\begin{array}{c}
m+1 \\
l+2
\end{array}\right) V^{(l+1)} \cdot \Theta^{(m-l)} \\
& =2^{m} \sum_{l=0}^{m-1}\left(\begin{array}{c}
m+1 \\
l+2
\end{array}\right)\left(\int V\right)^{(l+2)} \cdot\left(\Theta^{\prime}\right)^{(m+1)-(l+2)} \\
& =2^{m}\left[\left(\int V \cdot \Theta^{\prime}\right)^{(m+1)}-(m+1) \cdot V \cdot \Theta^{(m+1)}-\int V \cdot \Theta^{(m+2)}\right] \\
& =2^{m} \cdot\left(\int V \cdot \Theta^{\prime}\right)^{(m+1)} \quad \text { if } \quad \Theta^{(m+1)}=0 .
\end{aligned}
$$

Here we have used, in the second equality, the binomial identity

$$
\sum_{j=1}^{m-l} j \cdot\left(\begin{array}{c}
m-j \\
l
\end{array}\right)=\left(\begin{array}{c}
m+1 \\
l+2
\end{array}\right) \quad(\text { proof by induction on } m \text { ). }
$$

So $(\operatorname{ad} L)^{m+1}(\Theta)$ is of order $<m-1$ if and only if $(1.20)$ holds and $\int V \cdot \Theta^{\prime}$ is a polynomial of degree $\leqq m$, that is,

$$
V=\left(\frac{P}{\Theta^{\prime}}\right)^{\prime} \text { for some polynomial } P \text { of degree } m \text {. }
$$

This implies, in particular, that $V$ is rational, with at most $m-1$ poles, at the zeros of $\Theta^{\prime}$. At each pole $p$ the coefficient of $\frac{1}{x-p}$ in the Laurent expansion for $x \rightarrow p$ has to vanish, and the same is true for the coefficient of $\frac{1}{x}$ in the Laurent expansion of $V(x)$ for $x \rightarrow \infty$. These properties express the fact that the primitive $\int V$ of $V$ is rational as well.

The rationality of $V$ makes the differential equation (1.2) holomorphic on the entire Riemann sphere $\mathbb{C} \cup\{\infty\}$, having only finitely many singular points, each of which is of finite order. The further determination of $V$, in the next sections, will be carried out by studying the asymptotic behavior of the solutions at the singular points, starting at $\infty$, which is always an irregular singular point [unless $V(\infty)$ is finite and $\lambda=V(\infty)]$. 
However, before doing this, we conclude this section by mentioning some further partial results which can be obtained directly from the equation $(\operatorname{ad} L)^{m+1}(\Theta)=0$. Some of the lengthier proofs are only indicated, the later developments will not depend on them. These results, however, served as an indication for us as to which way to go. The equation $(\operatorname{ad} L)^{m+1}(\Theta)=0$ is equivalent to a system of polynomial equations in the coefficients of $\Theta$ and of the polynomial $P$ in (1.29). To solve this directly for arbitrary $m$ seems to be a hopeless task, because the complexity increases very rapidly with increasing $m$. See Sect. 7 for more comments on this.

Continuing the calculations of $(\operatorname{ad} L)^{m+1}(\Theta)$ in the way we obtained (1.29), one can prove that

$$
(\operatorname{ad} L)^{m+1}(\Theta) \text { is already of order } \leqq m-3 \text { if }(1.20),(1.29) \text { hold . }
$$

Moreover, its coefficient of $\partial_{x}^{m-3}$ is equal to zero if and only if

$$
\frac{1}{2} \Theta^{\prime \prime \prime} \cdot \int V-\frac{3}{2}\left(\int\left(V^{2}\right)\right) \cdot \Theta^{\prime}+\left(\frac{1}{2}\left(\int V\right)^{2} \cdot \Theta^{\prime}\right)^{\prime} \quad \text { is a polynomial of degree } \leqq m .
$$

A consequence of $(1.31)$ is that

$$
\int\left(V^{2}\right) \text {, like } \int V \text {, is rational. }
$$

For $\underline{m=1},(1.20),(1.29)$ imply that $V$ is a constant, which after a translation in $\lambda$ can be taken equal to zero. One verifies easily that the common solution space of (1.2), (1.3), where $A=A\left(\lambda, \partial_{\lambda}\right)$ is of first order and $\Theta=a x+b$ is one-dimensional and consists of the multiples of

$$
a_{ \pm}(k) e^{ \pm i k x}, \quad k^{2}=\lambda,
$$

where $a_{ \pm}(k)$ is a function of $k$ which determines $A$.

For $\underline{m=2}$, (1.20), (1.29) imply that, if $\operatorname{deg} \Theta=2$ :

$$
V(x)=\frac{c}{(x-a)^{2}}+b \quad(\text { Bessel potential })
$$

or $V(x)=\frac{c}{x^{2}}$ after a translation in $x$, respectively $\lambda$, over $a$, respectively $b$. If $a=b=0$ then $x \mapsto \psi(k x)$ is an eigenfunction for the eigenvalue $\lambda=k^{2}$ if and only if $\psi$ is an eigenfunction for the eigenvalue 1 , so it is obvious that we can interchange the role of $x$ and $k$ :

$$
\left(-\partial_{k}^{2}+\frac{c}{k^{2}}\right) \phi(x, k)=x^{2} \cdot \phi(x, k) \quad \text { if } \quad \phi(x, k)=\psi(k x) .
$$

If, on the other hand, $\operatorname{deg} \Theta=1$, then

$$
V(x)=\alpha \cdot x+\beta \quad(\text { Airy potential }) .
$$

Here a translation in $x$ by $\frac{\lambda}{\alpha}$ transforms an eigenfunction for the eigenvalue 0 to an eigenfunction for the eigenvalue $\lambda$, so again it is obvious that there is a second order equation (1.3) with $\Theta(x)=x$. In fact, the Airy potential can be obtained as the limit 
of the Bessel potential by taking

$$
c=\frac{1}{2} a^{3} \cdot \alpha, \quad b=\beta-\frac{1}{2} a \alpha \quad \text { and letting } a \rightarrow \infty .
$$

Both for the Bessel and the Airy potential, $A=A\left(\lambda, \partial_{\lambda}\right)$ therefore can be chosen such that the common solution space of (1.2) and (1.3) is two-dimensional.

For $\underline{m=3}$ it turns out that the equation $(\operatorname{ad} L)^{m+1}(\Theta)=0$ does not allow any new potentials beyond the Bessel and Airy ones. This can be seen for instance from (1.32) (which is not too hard to verify if $m=3$ ): if $V=\frac{c_{1}}{\left(x-a_{1}\right)^{2}}+\frac{c_{2}}{\left(x-a_{2}\right)^{2}}$, then $V^{2}$ has a term $\frac{1}{x-a_{1}} \cdot \frac{-2 c_{1} c_{2}}{\left(a_{1}-a_{2}\right)^{2}}$, so necessarily $c_{1}=0$ or $c_{2}=0$ if you want the primitive of $V^{2}$ to be rational.

For a while we thought that the Bessel and Airy potentials might be the only ones, but then a lengthy calculation showed that for $\underline{m=4}$ two new cases appear (normalized by translations in $x$, respectively $\lambda$ ):

Case $A$ :

$$
\begin{aligned}
V(x) & =\frac{2}{\left(x+t^{1 / 3}\right)^{2}}+\frac{2}{\left(x+\omega \cdot t^{1 / 3}\right)^{2}}+\frac{2}{\left(x+\omega^{2} t^{1 / 3}\right)^{2}} \\
& =-2 \partial_{x}^{2} \log \left(x^{3}+t\right), \quad \omega=e^{2 \pi i / 3},
\end{aligned}
$$

with the eigenfunction

$$
\phi(x, \lambda)=e^{i k x} \frac{x^{3}-3 x^{2} / i k-3 x / k^{2}+t}{x^{3}+t}, \quad k^{2}=\lambda,
$$

satisfying the differential equation

$$
\left[\left(-\partial_{k}^{2}+\frac{6}{k^{2}}\right)^{2}-4 t i \partial_{k}\right] \phi=\left(x^{4}+4 t x\right) \phi,
$$

the common solution space of (1.40) and (1.2) being one-dimensional.

Case B:

$$
V(x)=-\frac{1}{4} \frac{1}{x^{2}}+\frac{2}{(x+i \sqrt{t})^{2}}+\frac{2}{(x-i \sqrt{t})^{2}},
$$

with the eigenfunctions

$$
\phi(x, \lambda)=\psi^{\prime}(k x)-\frac{3 x^{2}-t}{2 k x\left(t+x^{2}\right)} \cdot \psi(k x), \quad k^{2}=\lambda,
$$

where $\psi$ is any solution of the Bessel equation

$$
\left(-\partial_{y}^{2}+\frac{3}{4} \frac{1}{y^{2}}\right) \psi(y)=\psi(y) .
$$

$\phi(x, \lambda)$ satisfies the differential equation

$$
\left[\left(-\partial_{k}^{2}+\frac{15}{4} \frac{1}{k^{2}}\right)^{2}+2 t\left(-\partial_{k}^{2}-\frac{1}{4} \frac{1}{k^{2}}\right)\right] \phi=\left(x^{4}+2 t x^{2}\right) \cdot \phi,
$$

and the common solution space of (1.44) and (1.2) is two-dimensional. 
Recognizing (1.38) as the first of the families of rational solutions of the $\mathrm{KdV}$ equation, as described in Airault et al. [3] and Adler and Moser [2], put us on the right track. Especially, when the next few families turned out to allow equations of the form (1.3), too, we became convinced that this could not be just a coincidence.

On the other hand, (1.41) certainly does not satisfy the $\mathrm{KdV}$ equations, which showed us in an early stage that "rational KdV" is not the full answer to the problem.

The potential (1.38) already appears in the paper of Bargmann [4] on reflectionless potentials. The existence of differential equations in $k$, however, is new even in this case.

\section{2. $V(\infty)$ is Finite}

Assume that $L=-\partial_{x}^{2}+V(x)$ and the non-constant $\Theta \equiv \Theta(x)$ satisfies $(\operatorname{ad} L)^{m+1}(\Theta)=0$. As we have seen in Sect. 1, this equation follows from the existence of a non-zero family of eigenfunctions $\phi(x, \lambda)$ of $L$ satisfying (1.3) for a suitable $m^{\text {th }}$ order linear differential operator $A=A\left(\lambda, \partial_{\lambda}\right)$. In (1.20), (1.29) we have seen that $\Theta$ is a polynomial of degree $\leqq m$ and $V$ is rational, of the form $V=\left(\frac{P}{\Theta^{\prime}}\right)^{\prime}$
for a polynomial $P$ of degree $\leqq m$.

We assume now also that $V(\infty)$ is finite, which certainly is true if $\operatorname{deg} \Theta=m$. By a translation in $\lambda$ we can arrange that $V(\infty)=0$, which implies that actually

$$
V(x)=O\left(\frac{1}{x^{2}}\right) \text { as } x \rightarrow \infty,
$$

because $\int V$ is rational. Writing

$$
\lambda=k^{2}, \quad k \in \mathbb{C}, \quad k \neq 0,
$$

the classical theory of irregular singular points applied to $x=\infty$ leads to the following results (see for instance Olver [19, Chap. 7]). Define the sectors

$$
\begin{aligned}
& S_{\infty}^{+}(k)=\{x \in \mathbb{C} ;|x| \text { large, } \varepsilon<\arg k+\arg x<\pi-\varepsilon\}, \\
& S_{\infty}^{-}(k)=\{x \in \mathbb{C} ;|x| \text { large },-\pi+\varepsilon<\arg k+\arg x<-\varepsilon\},
\end{aligned}
$$

where $\varepsilon>0$, but arbitrarily small. Then there are unique solutions $x \mapsto \phi_{\infty}^{ \pm}(x, k)$ of $\left(L-k^{2}\right) \phi \equiv 0$ with the asymptotic expansion

$$
\phi_{\infty}^{ \pm}(x, k) \sim e^{ \pm i k x} \sum_{j=0}^{\infty} a_{j}^{ \pm}(k) x^{-j} \quad \text { as } \quad|x| \rightarrow \infty \quad \text { in } \quad S_{\infty}^{ \pm}(k), \quad a_{0}(k)=1 .
$$

This expansion, due to Poincaré [20], can be termwise differentiated with respect to $x$. Expanding

$$
V(x) \sim \sum_{l=2}^{\infty} V_{l} \cdot x^{-l} \text { as } x \rightarrow \infty,
$$

(which is even a convergent power series in $1 / x$ for large $|x|$ because $V$ is rational) one can determine the $a_{j}^{ \pm}$recurrently by

$$
a_{p}^{ \pm}(k)=\frac{1}{ \pm 2 i k p}\left[(p-1) \cdot p \cdot a_{p-1}^{ \pm}-\sum_{l=2}^{p+1} V_{l} \cdot a_{p-(l-1)}^{ \pm}\right], \quad p \geqq 1 .
$$


So for each $p \geqq 1, a_{p}^{ \pm}(k)$ is a polynomial in $\frac{1}{k}$ of degree $\leqq p$, without a constant term. The coefficient of $\left(\frac{1}{k}\right)^{p}$ is equal to

$$
a_{p, p}^{ \pm}=\prod_{j=1}^{p}\left(j(j-1)-V_{2}\right) /\left(p !( \pm 2 i)^{p}\right) .
$$

The asymptotic expansion (2.4) can now be termwise differentiated with respect to both variables $(x, k)$, and is actually valid for the analytic continuations of $x \mapsto \phi_{\infty}^{ \pm}(x, k)$ in the larger sectors,

$$
\begin{aligned}
& \tilde{S}_{\infty}^{+}(k)=\{x \in \mathbb{C} ;|x| \text { large, }-\pi+\varepsilon<\arg k+\arg x<2 \pi-\varepsilon\}, \\
& \tilde{S}_{\infty}^{-}(k)=\{x \in \mathbb{C} ;|x| \text { large, }-2 \pi+\varepsilon<\arg k+\arg x<\pi-\varepsilon\} .
\end{aligned}
$$

One of the confusing facts here is that $\tilde{S}_{\infty}^{ \pm}(k)$ overlap themselves and the analytic continuation of $\phi_{\infty}^{ \pm}(x, k)$ for a growing argument of $x$ does not necessarily coincide with the analytic continuation for decreasing argument. (Another confusing fact being that the sectors move along as $k$ turns around the origin.)

Now one can choose to continue $x \mapsto \phi_{\infty}^{+}(x, k)$ analytically for decreasing argument of $x$ and $x \mapsto \phi_{\infty}^{-}(x, k)$ for increasing argument to the single valued analytic functions

$$
x \mapsto{ }^{0} \phi_{\infty}^{+}(x, k), \quad \text { respectively } \quad x \mapsto{ }^{0} \phi_{\infty}^{-}(x, k),
$$

both defined in the sector

$$
S_{\infty}^{0}(k)=\{x \in \mathbb{C} ;|x| \text { large, }-\pi+\varepsilon<\arg k+\arg x<\pi-\varepsilon\} .
$$

Because ${ }^{0} \phi_{\infty}^{+}(x, k)$ is exponentially increasing as $|x| \rightarrow \infty$ in $S_{\infty}^{-}$and ${ }^{0} \phi_{\infty}^{-}(x, k)$ is exponentially decreasing there, ${ }^{0} \phi_{\infty}^{+}$and ${ }^{0} \phi_{\infty}^{-}$are linearly independent and therefore form a basis of solutions of $\left(L-k^{2}\right) \phi=0$.

Continuing $x \mapsto \phi_{\infty}^{+}(x, k)$, respectively $x \mapsto \phi_{\infty}^{-}(x, k)$ analytically for increasing, respectively decreasing, argument of $x$, one obtains single valued analytic functions

$$
x \mapsto{ }^{\pi} \phi_{\infty}^{+}(x, k), \quad \text { respectively } \quad x \mapsto{ }^{\pi} \phi_{\infty}^{-}(x, k),
$$

defined in

$$
S_{\infty}^{\pi}(k)=\{x \in \mathbb{C} ;|x| \text { large, } \varepsilon<\arg k+\arg x<2 \pi-\varepsilon\} .
$$

Clearly, $S_{\infty}^{0}(k) \cup S_{\infty}^{\pi}(k)$ is a full (punctured) neighborhood of $\infty$ on the Riemann sphere $\mathbb{C} \cup\{\infty\}$. On the other hand, $S_{\infty}^{0}(k) \cap S_{\infty}^{\pi}(k)$ has two connected components, the $S_{\infty}^{+}(k)$, respectively $S_{\infty}^{-}(k)$ of $(2.3)$. Using that ${ }^{0} \phi_{\infty}^{+},{ }^{0} \phi_{\infty}^{-}$and ${ }^{\pi} \phi_{\infty}^{+}, \pi{ }_{\infty}^{-}$are a basis of $\operatorname{Ker}\left(L-k^{2}\right)$, and using the validity of (2.4) in the larger sectors $\tilde{S}_{\infty}^{ \pm}(k)$, it follows that there are constants $\sigma_{\infty}^{ \pm}(k) \in \mathbb{C}$ such that

$$
\begin{aligned}
& { }^{0} \phi_{\infty}^{-}(x, k)={ }^{\pi} \phi_{\infty}^{-}(x, k)+\sigma_{\infty}^{+}(k) \cdot \phi_{\infty}^{+}(x, k) \text { in } S_{\infty}^{+}(k), \\
& { }^{\pi} \phi_{\infty}^{+}(x, k)={ }^{0} \phi_{\infty}^{+}(x, k)+\sigma_{\infty}^{-}(k) \cdot \phi_{\infty}^{-}(x, k) \text { in } S_{\infty}^{-}(k) .
\end{aligned}
$$

$\sigma_{\infty}^{ \pm}(k)$ are the so-called Stokes multipliers for the irregular singular point $\infty$. There is a partial relationship with the monodromy $M_{\infty}^{(x)}=$ analytic continuation of 
solutions as $x$ turns around clockwise with large $|x|$. Indeed, on the basis of ${ }^{0} \phi_{\infty}^{+}(\cdot, k),{ }^{0} \phi_{\infty}^{-}(\cdot, k)$ in $S_{\infty}^{0}(k)$, the second relation in (2.13) gives

$$
M_{\infty}^{(x)}=\left(\begin{array}{cc}
1 & c(k) \\
\sigma_{\infty}^{-}(k) & d(k)
\end{array}\right),
$$

for some $c(k), d(k) \in \mathbb{C}$. Because the Wronski determinant is constant, $\operatorname{det} M_{\infty}^{(x)}=1$, so we also know that

$$
d(k)=1+c(k) \cdot \sigma_{\infty}^{-}(k) .
$$

Now let $\mathscr{Q}^{ \pm}$be the space of functions of $x$ which are bounded in the sector $S_{\infty}^{ \pm}(k)$, keeping $k$ close to a fixed $k_{0} \neq 0$. The termwise differentiability of (2.4) with respect to $k$ shows that

$$
\partial_{k}^{j}{ }^{0} \phi_{\infty}^{ \pm}(\cdot, k) \in \mathscr{Q}^{ \pm} \quad \text { for all } j \geqq 0 .
$$

The analogous asymptotics for the differential operator $\left(L-k^{2}\right)^{m+1}$ of order $2(m+1)$ yields that

$$
\operatorname{dim}\left(\operatorname{Ker}\left(L-k^{2}\right)^{m+1} \cap \mathscr{Q}^{ \pm}\right)=m+1 .
$$

Because $\Theta$ is a polynomial, $\Theta \cdot{ }^{0} \phi_{\infty}^{ \pm}(\cdot, k) \in \mathscr{2}^{ \pm}$, we can apply Lemma 1.3 to conclude that

Lemma 2.1. There are unique linear differential operators $B^{ \pm}\left(k, \partial_{k}\right)$ of order $\leqq m$ in $k$, with coefficients depending on $k$, such that

$$
B^{ \pm}\left(k, \partial_{k}\right){ }^{0} \phi_{\infty}^{ \pm}(x, k)=\Theta(x) \cdot{ }^{0} \phi_{\infty}^{ \pm}(x, k),
$$

and a similar conclusion holds with ${ }^{0} \phi_{\infty}^{ \pm}$replaced $b y^{\pi} \phi_{\infty}^{ \pm}$.

Corollary 2.2. Let $x \mapsto \phi(x, k)$ be a non-zero family of solutions of $\left(L-k^{2}\right) \phi=0$ and $B=B\left(k, \partial_{k}\right)$ a linear differential operator of order $\leqq m$ such that

$$
B\left(k, \partial_{k}\right) \phi(x, k)=\Theta(x) \cdot \phi(x, k) .
$$

Then $B$ is conjugate to either $B^{+}$or $B^{-}$by means of a non-zero function of $k$. If $B^{+}$is not conjugate to $B^{-}$by means of a non-zero function of $k$, then $\phi$ is necessarily of the form $c(k) \cdot{ }^{0} \phi_{\infty}^{+}(x, k)$ or $c(k) \cdot{ }^{0} \phi_{\infty}^{-}(x, k)$ for some function $c$ of $k$. In this case, the space of common local solutions of (2.19) and $\left(L-k^{2}\right) \phi=0$ is one-dimensional. If $B^{+}$ is conjugate to $B^{-}$by means of a function of $k$ then the space of common local solutions is two-dimensional.

Proof. Because ${ }^{0} \phi_{\infty}^{+}(\cdot, k),{ }^{0} \phi_{\infty}^{-}(\cdot, k)$ is a basis of $\operatorname{Ker}\left(L-k^{2}\right)$, we have

$$
\phi(x, k)=c_{+}(k) \cdot{ }^{0} \phi_{\infty}^{+}(x, k)+c_{-}(k) \cdot{ }^{0} \phi_{\infty}^{-}(x, k)
$$

for suitable functions $c_{+}, c_{-}$of $k$. Now write

$$
\begin{aligned}
B c_{+} \phi^{+}+B c_{-} \phi^{-} & =B \phi=\Theta \phi=\Theta c_{+} \phi^{+}+\Theta c_{-} \phi^{-} \\
& =c_{+} \Theta \phi^{+}+c_{-} \Theta \phi^{-}=c_{+} B^{+} \phi^{+}+c_{-} B^{-} \phi^{-} .
\end{aligned}
$$

Now

$$
\begin{gathered}
B c_{+} \phi^{+}-c_{+} B^{+} \phi^{+} \in \operatorname{Ker}\left(L-k^{2}\right)^{m+1} \cap \mathscr{Q}^{+}, \quad c_{-} B^{-} \phi^{-}-B c_{-} \phi^{-} \\
\in \operatorname{Ker}\left(L-k^{2}\right)^{m+1} \cap \mathscr{Q}^{-}
\end{gathered}
$$


and these latter spaces are complementary; we conclude from $B c_{+} \phi^{+}-c_{+} B^{+} \phi^{+}$ $=c_{-} B^{-} \phi^{-}-B c_{-} \phi^{-}$, that $B c_{+} \phi^{+}=c_{+} B^{+} \phi^{+}, c_{-} B^{-} \phi^{-}=B c_{-} \phi^{-}$.

Because the eigenvalue parameter $\Theta(x)$ in $(2.18)$ is not constant as function of $x$, the space of functions of $k$, spanned by the functions $k \rightarrow{ }^{0} \phi_{\infty}^{ \pm}(x, k)$, with varying $x$, is infinite-dimensional. So in the same way as we proved (1.9) $\Rightarrow(1.10)$ it follows that $B \cdot c_{+}=c_{+} \cdot B^{+}$and $c_{-} \cdot B^{-}=B \cdot c_{-}$. Because $c_{+}, c_{-}$are not both equal to zero, the first conclusion in the corollary follows. If $c_{+}, c_{-}$are both non-zero, then $B^{+}=c_{+}^{-1} \cdot B \cdot c_{+}=\left(c_{+}^{-1} \cdot c_{-}\right) \cdot B^{-} \cdot\left(c_{-}^{-1} \cdot c_{+}\right)$, proving the second statement. Now let $\tilde{\phi}=\tilde{c}_{+}(k) \phi^{+}+\tilde{c}_{-}(k) \phi^{-} \neq 0$ be any other common local solution. If $B$ is conjugate to $B^{+}$by a function, but not to $B^{-}$, then $\tilde{c}_{-}=0, \tilde{c}_{+} \neq 0, B \cdot \tilde{c}_{+}=\tilde{c}_{+} \cdot B^{+}$. Together with $B \cdot c_{+}=c_{+} \cdot B^{+}$we get that $B^{+}$commutes with $c_{+} / \tilde{c}_{+}$. Because the order of $B^{+}$is positive, this implies that $c_{+} / \tilde{c}_{+}$is a constant, as is well-known. If $B^{+}$ is conjugate to $B^{-}$by a function, then $B$ is conjugate to both $B^{+}$and $B^{-}$by some functions. In this case it is allowed that $\tilde{c}_{+} \neq 0$ and $\tilde{c}_{-} \neq 0$, but the same argument as above gives that $\tilde{c}_{+}$, respectively $\tilde{c}_{-}$is a constant multiple of $c_{+}$, respectively $c_{-}$. This proves the last two statements.

Now, writing

$$
\begin{gathered}
B^{ \pm}\left(k, \partial_{k}\right)=\sum_{p=0}^{m} B_{p}^{ \pm}(k) \partial_{k}^{p}, \\
\Theta(x)=\sum_{r=0}^{m} \Theta_{r} \cdot x^{r},
\end{gathered}
$$

the substitution of (2.4) in (2.18) yields, collecting the coefficients of $x^{q}$ :

$$
\sum_{\substack{j, s \\
j \geqq 0,0 \leqq s \leqq m}} B_{q+s+j}^{ \pm}(k) \cdot\left(\begin{array}{c}
q+s+j \\
s
\end{array}\right) \cdot( \pm i)^{q+j} \cdot \partial_{k}^{s} a_{j}^{ \pm}(k)=\sum_{r=0}^{m} a_{r-q}^{ \pm}(k) \cdot \Theta_{r} .
$$

Because $a_{0}^{ \pm}(k)=1$, Eqs. (2.22) from $q=m, m-1$, downwards to $q=0$, determine $B_{m}^{ \pm}, B_{m-1}^{ \pm}, \ldots, B_{0}^{ \pm}$successively. For $q=m$ we get

$$
B_{m}^{ \pm}(k)=( \pm i)^{-m} \cdot \Theta_{m} .
$$

Assuming that $B\left(k, \partial_{k}\right)$ is truly of order $m$, that is, $B_{m}(k) \neq 0$, it follows that $\Theta_{m} \neq 0$. In other words, the finiteness of $V(\infty)$ is equivalent to the property that the order of $B$ is equal to the degree of $\Theta$.

Furthermore, $B_{m}^{ \pm}$is a (non-zero) constant and recalling that $a_{j}^{ \pm}$is a polynomial in $\frac{1}{k}$ of degree $\leqq j$, one gets by downward induction on $p$ :

$$
B_{p}^{ \pm}(k) \text { is a polynomial in } \frac{1}{k} \text { of degree } \leqq m-p .
$$

It follows that Eq. (2.18) also extends with respect to $k$ to the Riemann sphere $\mathbb{C} \cup\{\infty\}$ with singular points only at $k=0$ and $k=\infty$, both of finite order. At $k=0$ the singular point is regular and, for the generic value of $x$, the singularity at $k=\infty$ is irregular.

The common solutions $\phi$ of (2.18) for $B^{+}$, say, and $\left(L-k^{2}\right) \phi=0$ have a unique analytic continuation along any curve $\gamma$ in the complex two-dimensional domain

$$
D=\{(x, k) \in \mathbb{C} \times \mathbb{C} ; x \text { is not a pole of } V \text { and } k \neq 0\} .
$$


For $(x, k) \in D$, let $\mathscr{S}_{(x, k)}$ be the space of germs at $(x, k)$ of common solutions of $(2.18)$ and $\left(L-k^{2}\right) \phi=0$. Then Corollary 2.2 can be phrased differently, saying that the $\mathscr{S}_{(x, k)},(x, k) \in D$ form a holomorphic vector bundle $\mathscr{S}$ over $D$ of complex dimension 1 or 2 , the analytic continuation of solutions defining a flat holomorphic connection in $\mathscr{S}$. A priori the system of differential equations $\left(L-k^{2}\right) \phi=0$, $(B-\Theta(x)) \phi=0$ makes that $\phi$ is uniquely determined by the vector $w(x, k)$ $=\left(\partial_{x}^{i} \partial_{k}^{j} \phi(x, k)\right), i=0,1, j=0,1, \ldots, m-1$ at any point $(x, k)$ in $D$. It satisfies two differential equations of the form $\partial_{x} w=R(x, k) w, \partial_{k} w=S(x, k) w$. The compatibility conditions (of curvature type) apparently cut down the dimension of the solution space from $2 m$ to one or two.

The last generalities of this section concern the asymptotics for $k \rightarrow \infty$. Writing

$$
\begin{aligned}
a_{j}^{ \pm}(k) & =\sum_{s=0}^{j} a_{j, s}^{ \pm} \cdot k^{-s}, \\
B_{p}^{ \pm}(k) & =\sum_{s=0}^{m-p} B_{p, s}^{ \pm} \cdot k^{-s},
\end{aligned}
$$

we can compare the powers of $\frac{1}{k}$ in (2.22). The constant term yields

$$
B_{q, 0}^{ \pm}( \pm i)^{q}=\Theta_{q} \text { for all } q \text {, that is } \sum_{q=0}^{m} B_{q, 0}^{ \pm}( \pm i x)^{q}=\Theta(x) .
$$

Comparing the coefficients of $k^{-1}$, using that $k^{-1}$ never occurs as $\partial_{k}^{s} k^{-l}$ for an integer $l$ (unless $s=0, l=1$ ), we get

$$
\sum_{j} B_{q+j, 0}^{ \pm} \cdot( \pm i)^{q+j} a_{j, 1}+B_{q, 1}^{ \pm} \cdot( \pm i)^{q}=\sum_{r=0}^{m} a_{r-q, i} \cdot \Theta_{r},
$$

which, using (2.28), simply amounts to

$$
B_{q, 1}^{ \pm}=0 \text { for all } q .
$$

This means that (2.18) is a perturbation of a constant coefficient equation by terms of order $O\left(\frac{1}{k^{2}}\right)$. If $t=\Theta(x)$ is a regular value of $\Theta$, that is

$$
\Theta\left(x_{j}\right)=t \text { for } m \text { different } \quad x_{j} \in \mathbb{C},
$$

then (2.28) gives that the unperturbed equation has a basis of solutions (considered as functions of $k$ ) given by

$$
e^{ \pm i x_{j} \cdot k}, \quad j=1, \ldots, m .
$$

Choosing $|x|$ so large that $x$ becomes extremal among the $x_{j}$, the aforementioned theory of irregular singular points applied to (2.18) yields that $\phi^{ \pm}$also has an asymptotic expansion of the form

$$
\phi^{ \pm}(x, k) \sim e^{ \pm i k x} \sum_{l=0}^{\infty} \alpha_{l}^{ \pm}(x) k^{-l} \text { as } k \rightarrow \infty,
$$

in a direction in which $e^{ \pm i k x}$ decreases fastest among the $e^{ \pm i k x_{j}}$. Comparing (2.4) and (2.32) yields

$$
\alpha_{l}^{ \pm}(x)=\sum_{j \geqq l} a_{j, l} \cdot x^{-j},
$$


the convergence of which, for $|x|$ sufficiently large, can be obtained from (2.6). The result is absolute convergence if $|x|>|p|$ for all poles $p$ of $V$.

An expansion of the form (2.32) is called a Bloch function, Baker function, or WKB-approximation, depending on the background of the author. Because the expansion can be differentiated termwise with respect to $x$, the equation $\left(L-k^{2}\right) \phi=0$ leads to

$$
\pm 2 i \partial_{x} \alpha_{l+1}^{ \pm}(x)=-\partial_{x}^{2} \alpha_{l}^{ \pm}(x)+V(x) \alpha_{l}^{ \pm}(x) .
$$

Because also

$$
\alpha_{l+1}^{ \pm}(x) \rightarrow 0 \text { for } x \rightarrow \infty, \text { for all } l \geqq 0, \quad \alpha_{0}^{ \pm}(x) \equiv 1,
$$

the $\alpha_{l}^{ \pm}$can be recursively determined by simple integrations. The first few are

$$
\begin{gathered}
\alpha_{1}^{ \pm}(x)=\frac{1}{ \pm 2 i}\left(\int V\right)(x), \\
\alpha_{2}^{ \pm}=\frac{1}{4} V-\frac{1}{8}\left(\int V\right)^{2}, \\
\pm 2 i \cdot \alpha_{3}^{ \pm}=-\frac{1}{4} V^{\prime}+\frac{1}{4} \int V \cdot V-\frac{1}{24}\left(\int V\right)^{3}+\frac{1}{4} \int V^{2} .
\end{gathered}
$$

On the other hand, Eq. (2.18) yields

$$
\begin{aligned}
\Theta(x) \cdot \alpha_{l}^{ \pm}(x)= & \sum_{j} B_{j, l}^{ \pm} \cdot( \pm i x)^{j} \cdot \alpha_{0}^{ \pm}(x)+\sum_{\substack{j, J, L \\
0 \leqq J \leqq j \leqq m, L \geqq 1}} B_{j, l-L-J}^{ \pm} \cdot\left(\begin{array}{l}
j \\
J
\end{array}\right) \cdot( \pm i x)^{j-J} \\
& \cdot \alpha_{L}^{ \pm}(x) \cdot(-1)^{J} \frac{(L+J-1) !}{(L-1) !}
\end{aligned}
$$

for all $l \geqq 0$. The equation for $l=0$ is just (2.28). This makes the terms on the righthand side of (2.39) for $J=0, L=l$ drop out against the left-hand side. Introducing the notation

$$
\beta_{p}^{ \pm}(x)=\sum_{j} B_{j, p}^{ \pm} \cdot( \pm i x)^{j},
$$

(2.39) can be written in a somewhat more compact form:

$$
\beta_{l}^{ \pm}(x)+\sum_{\mu \geqq 1} \sum_{0 \leqq p \leqq \mu}( \pm i)^{\mu-p} \cdot \partial_{x}^{\mu-p} \beta_{p}^{ \pm}(x) \cdot \alpha_{l-\mu}^{ \pm}(x) \cdot\left(\begin{array}{c}
l-p-1 \\
\mu-p
\end{array}\right)=0 .
$$

Because $\beta_{1}^{ \pm}=0$ by (2.29) we see that (2.41) determine the $\alpha_{r}^{ \pm}$successively because the coefficient of $\alpha_{l-1}^{ \pm}(x)$ in $(2.41)$ is $\pm i \cdot \partial_{x} \beta_{0}^{ \pm}(x) \cdot(l-1)= \pm i \cdot(l-1) \cdot \Theta^{\prime}(x)$. By induction we get

$$
\alpha_{r}^{ \pm}(x)=\frac{P_{r}^{ \pm}(x)}{\left(\Theta^{\prime}(x)\right)^{r}} \text { for some polynomial } P_{r}^{ \pm} .
$$

In particular, each $\alpha_{r}^{ \pm}, r \geqq 1$, is rational with poles at most at the zeros of $\Theta^{\prime}$. From (2.36) we recover that $\int \bar{V}$ is rational, and then from (2.38) that $\int V^{2}$ is rational as well; compare (1.32).

\section{The Rational KdV Potentials}

Let $L=-\partial_{x}^{2}+V(x)$ and $\Theta=\Theta(x)$ satisfy $(\operatorname{ad} L)^{m+1}(\Theta)=0$. As in Sect. 2 we assume that $\operatorname{deg} \Theta=m$, so we may assume that $V(\infty)=0$. However, we will restrict 
ourselves now to the case that the operators $B^{+}$and $B^{-}$in (2.18) are not conjugate to each other by a non-zero function of $k$.

Lemma 3.1. The finite singular points of the equation $\left(L-k^{2}\right) \phi=0$ are regular, that is

$$
V(x) \sim \frac{c_{-2}}{(x-p)^{2}} \text { as } x \rightarrow p \text { for each pole } p \in \mathbb{C} \text { of } V .
$$

Proof. Suppose that $p \in \mathbb{C}$ is a higher order pole of $V$ :

$$
V(x)=\sum_{r \geqq \mu} c_{r}(x-p)^{r}, \quad \text { with } \quad c_{\mu} \neq 0, \quad \mu<-2 .
$$

The theory of irregular singular points (cf. Olver [19, Chap. 7]) this time looks as follows. Choose a square root $c_{\mu}^{1 / 2}$ of $c_{\mu}$. For each integer $j$ define the sectors

$$
\begin{aligned}
S_{p, j}^{+}= & \{x \in \mathbb{C} ;|x-p| \text { small, } \\
& \left.\varepsilon+2 \pi j<\arg c_{\mu}^{1 / 2}+\left(1+\frac{\mu}{2}\right) \arg (x-p)<\pi-\varepsilon+2 \pi j\right\}, \\
S_{p, j}^{-}= & \{x \in \mathbb{C} ;|x-p| \text { small, } \\
& \left.-\pi+\varepsilon+2 \pi j<\arg c_{\mu}^{1 / 2}+\left(1+\frac{\mu}{2}\right) \arg (x-p)<-\varepsilon+2 \pi j\right\} .
\end{aligned}
$$

Then there are unique solutions $x \mapsto \phi_{p, j}^{ \pm}(x, k)$ of $\left(L-k^{2}\right) \phi=0$, with the asymptotic expansion

$$
\begin{aligned}
\phi_{p, j}^{ \pm}(x, k) \sim \exp & {\left[ \pm \sum_{0 \leqq l<-\left(\frac{\mu}{2}+1\right)} A_{l} \cdot(x-p)^{\frac{\mu}{2}+1+l}\right] \cdot(x-p)^{\omega^{ \pm}} } \\
& \cdot \sum_{r \geqq 0} d_{r}^{ \pm}\left(k^{2}\right) \cdot(x-p)^{r / 2} \quad \text { as } x \rightarrow p \text { in } S_{p, j}^{ \pm} .
\end{aligned}
$$

Here $A_{0}=c_{\mu}^{1 / 2} /\left(\frac{\mu}{2}+1\right)$, the other $A_{l}$ depend neither on $\lambda=k^{2}$ nor on the choice of the sign. $\omega^{ \pm}$does not depend on $k^{2}, \omega^{+}+\omega^{-}=-\frac{\mu}{2}$; if $\mu$ is odd then in fact $\omega^{+}=\omega^{-}=\frac{-\mu}{4}$. Finally, $d_{0}=1$ (normalization convention) and the $d_{r}$ for $r<-\mu+2$ do not depend on $k^{2}$, the others are polynomials in $k^{2}$ of degree $\leqq r /(-\mu+2)$. Again, the expansion (3.4) actually remains valid for the analytic continuation of $x \mapsto \phi_{p, j}^{ \pm}(x, k)$ to the larger sectors,

$$
\begin{aligned}
\tilde{S}_{p, j}^{+}= & \{x \in \mathbb{C} ;|x-p| \text { small, } \\
& \left.-\pi+\varepsilon+2 \pi j<\arg c_{\mu}^{1 / 2}+\left(1+\frac{\mu}{2}\right) \arg (x-p)<2 \pi-\varepsilon+2 \pi j\right\}, \\
\tilde{S}_{p, j}^{-}= & \{x \in \mathbb{C} ;|x-p| \text { small, } \\
& \left.-2 \pi+\varepsilon+2 \pi j<\arg c_{\mu}^{1 / 2}+\left(1+\frac{\mu}{2}\right) \arg (x-p)<\pi-\varepsilon+2 \pi j\right\},
\end{aligned}
$$

and in the overlaps the two solutions form a basis of $\operatorname{Ker}\left(L-k^{2}\right)$. Also the expansion (3.4) can be termwise differentiated with respect to $x$ and $k$. 
Now let $\mathscr{2}_{p, j}^{ \pm}$be the space of functions of $x$ which converge to 0 as $x \rightarrow p$ in the sector $S_{p, j}^{ \pm}$. Then

$$
\operatorname{dim} \operatorname{Ker}\left(L-k^{2}\right)^{m+1} \cap \mathscr{Q}_{p, j}^{ \pm}=m+1
$$

To see this, write $\phi(x, k)=\psi(t, k)$ with $t=c_{\mu}^{1 / 2}(x-p)^{\frac{\mu}{2}+1} /\left(\frac{\mu}{2}+1\right)$. Then

$$
L-k^{2}=c_{\mu}(x-p)^{\mu}\left[-\partial_{t}^{2}+O\left(t^{-1}\right) \partial_{t}+1+O\left(t^{-1}\right)\right],
$$

so (3.6) follows from a classical perturbation argument for $t \rightarrow \infty$. Because obviously

$$
\partial_{k}^{j} \phi_{p, j}^{ \pm}(\cdot, k) \in \mathscr{Q}^{ \pm}, \quad \Theta \cdot \phi_{p, j}^{ \pm}(\cdot, k) \in \mathscr{Q}^{ \pm},
$$

we can apply Lemma 1.3 to conclude that there are linear differential operators $B_{p, j}^{ \pm}\left(k, \partial_{k}\right)$ such that

$$
B_{p, j}^{ \pm}\left(k, \partial_{k}\right) \phi_{p, j}^{ \pm}(x, k)=\Theta(x) \cdot \phi_{p, j}^{ \pm}(x, k) .
$$

The assumption that $B_{\infty}^{+}$and $B_{\infty}^{-}$are not conjugate to each other now implies (Corollary 2.2) that $\phi_{p, j}^{ \pm}$is a multiple of (the analytic continuation of) either $\phi_{\infty}^{+}$or $\phi_{\infty}^{-}$. By a suitable choice of the square root of $c_{\mu}$ we can arrange that

for some function $c$ of $k$.

$$
\phi_{p, j}^{+}(x, k)=c(k) \cdot \phi_{\infty}^{+}(x, k)
$$

Now let $\gamma(t)=(x(t), k(t)), t$ running from 0 to 1 , be a curve in the domain $D$ of (2.25), such that $|x(t)|$ remains large and $\arg k(t)+\arg x(t) \equiv \frac{\pi}{2}$, so that $x(t) \in S_{\infty}^{+}(k(t))$ [see (2.3)], for all $t$. If $k(0)=k, k(1)=-k$, then the analytic continuation of $\phi_{\infty}^{+}$ along $\gamma$ arrives at another solution of $\left(L-k^{2}\right)$, which apparently is $\phi_{\infty}^{-}$, and which is a solution of $\left(B_{\infty}^{-}-\Theta\right) \phi=0$. Now $\gamma$ is homotopic to $\delta=$ keeping $x$ constant and letting $k$ in turn around the origin to $-k$, followed by $\varepsilon=$ keeping $-k$ fixed and letting $x$ turn in the opposite direction. Since analytic continuation along $\varepsilon$ keeps us in $\operatorname{Ker}\left(L-k^{2}\right) \cap \operatorname{Ker}\left(B_{\infty}^{-}-\Theta\right) \phi$, the conclusion is that analytic continuation along $\delta$ maps

$$
\operatorname{Ker}\left(L-k^{2}\right) \cap \operatorname{Ker}\left(B_{\infty}^{+}-\Theta\right) \quad \text { into } \operatorname{Ker}\left(L-k^{2}\right) \cap \operatorname{Ker}\left(B_{\infty}^{-}-\Theta\right)
$$

However, the sectors $S_{p, j}^{ \pm}$do not depend on $k$ and it is easily seen that $\phi_{p, j}^{+}$is invariant under analytic continuation along $\delta$. Because (3.10) is preserved under analytic continuation, we arrive at a contradiction.

Lemma 3.2. Around each finite pole p of $V$, all eigenfunctions of L are single-valued.

Proof. At a regular singular point $p$, that is, when (3.2) holds with $\mu=2$, one has the following possibilities.

(A) If $c_{-2}=l^{2}-\frac{1}{4}$ with $2 l \notin \mathbb{Z}$, then $\left(L-k^{2}\right) \phi=0$ has a basis of solutions

$$
\phi_{p}^{ \pm}(x, k)=(x-p)^{\frac{1}{2} \pm l} \sum_{r=0}^{\infty} d_{r}^{ \pm}\left(k^{2}\right) \cdot(x-p)^{r}, \quad d_{0}=1
$$

where the power series has a positive radius of convergence. The coefficients $d_{r}$ are determined by the recurrent relations [obtained from the equation $\left.\left(L-k^{2}\right) \phi_{p}^{ \pm}=0\right]$ :

$$
r(r \pm 2 l) d_{r}^{ \pm}=\sum_{s=-1}^{r-2} \check{c}_{s} \cdot d_{r-2-s}^{ \pm}, \quad r \geqq 1,
$$


where we have written $\check{c}_{s}=c_{s}$ if $s \neq 0, \check{c}_{0}=c_{0}-k^{2}=c_{0}-\lambda$. It follows by induction that $d_{2 j}$ is a polynomial in $\lambda=k^{2}$ of degree $\leqq j$, as well as $d_{2 j+3}$, for $j \in \mathbb{Z}_{\geqq 0}\left(d_{1}=0\right)$. The series (3.11) can be termwise differentiated, also with respect to $k$.

Now let $\mathscr{Q}_{p}^{ \pm}$be the space of multi-valued holomorphic functions of $x$ in a punctured neighborhood of $p$, the analytic continuation of which, after turning around $p$ once, gets multiplied by $e^{2 \pi i\left(\frac{1}{2} \pm l\right)}$. Clearly,

$$
\partial_{k}^{j} \phi_{p}^{ \pm}(\cdot, k) \in \mathscr{Q}_{p}^{ \pm}, \quad \Theta \cdot \phi_{p}^{ \pm}(\cdot, k) \in \mathscr{Q}_{p}^{ \pm},
$$

so, in particular,

$$
\operatorname{dim}\left(\operatorname{Ker}(L-\lambda)^{m+1} \cap \mathscr{Q}_{p}^{+}\right) \geqq m+1, \quad \operatorname{dim} \operatorname{Ker}\left((L-\lambda)^{m+1} \cap \mathscr{Q}_{p}^{-}\right) \geqq m+1 .
$$

Now $\mathscr{Q}_{p}^{+} \cap \mathscr{Q}_{p}^{-}=0\left(e^{2 \pi i\left(\frac{1}{2}+l\right)} \neq e^{2 \pi i\left(\frac{1}{2}-l\right)}\right.$ because $\left.2 l \notin \mathbb{Z}\right)$ implies that equality has to hold in (3.14). So we can apply Lemma 1.3 to conclude that there are linear differential operators $B_{p}^{ \pm}\left(k, \partial_{k}\right)$ such that

$$
B_{p}^{ \pm}\left(k, \partial_{k}\right) \phi_{p}^{ \pm}(x, k)=\Theta(x) \cdot \phi_{p}^{ \pm}(x, k) .
$$

Now as in the last part of the proof of Lemma 3.1 we get a contradiction once again because $\phi_{p}^{ \pm}(x, k)$ remains unchanged if $k$ is moved to $-k$.

(B) Now assume that $c_{-2}=l^{2}-\frac{1}{4}$ with $2 l \in \mathbb{Z}_{\geqq 0}$. In this case $\phi_{p}^{+}$of (3.11) survives as a solution, called the recessive solution. However, $\phi_{p}^{-}$has to be replaced by

$$
\tilde{\phi}_{p}^{-}(x, k)=(x-p)^{\frac{1}{2}-l} \sum_{r=0}^{\infty} d_{r}^{-}\left(k^{2}\right) \cdot(x-p)^{r}+c(k) \cdot \log (x-p) \cdot \phi_{p}^{+}(x, k) .
$$

Here the $d_{r}^{-}$for $1 \leqq r \leqq 2 l$ are determined as before by (3.12). Furthermore,

$$
2 l \cdot c(k)=\sum_{s=-1}^{2 l-2} \check{c}_{s} \cdot d_{2 l-2-s}^{-} .
$$

$d_{2 l}^{-}$can be chosen arbitrarily (reflecting the fact that one can add an arbitrary multiple of $\phi_{p}^{-}$to $\tilde{\phi}_{p}^{-}$), and $\tilde{d}_{r}^{-}$for $r>2 l$ are then determined by

$$
r(r-2 l) d_{r}^{-}=\sum_{s=-1}^{r-2} \check{c}_{s} \cdot d_{r-2-s}-c(k) \cdot 2(l+r) \cdot d_{r}^{+} .
$$

If $\phi(x, k)$ is any non-zero solution of $\left(L-k^{2}\right) \phi=0,\left(B\left(k, \partial_{k}\right)-\Theta(x)\right) \phi=0$, then either $\phi(x, k)=\alpha(k) \cdot \phi_{p}^{+}(x, k)$ or $\phi(x, k)=\beta(k) \cdot \widetilde{\phi}_{p}^{-}(x, k)$ for a $\widetilde{\phi}_{p}^{-}$as described above. In the second case, going with $x$ around $p$ and using that $\Theta$ is single-valued around $p$, we get, apart from the equation $(B-\Theta) \cdot \beta \tilde{\phi}_{p}^{-}=0$, also the equation $(B-\Theta)$ $\cdot \beta\left(e^{2 \pi i\left(\frac{1}{2} \pm l\right)} \tilde{\phi}_{p}^{-}+2 \pi i c(k) \phi_{p}^{+}\right)=0$. Multiplying the first one with $e^{2 \pi i\left(\frac{1}{2} \pm l\right)}$ and subtracting it from the second one, we see that $(B-\Theta) \cdot \alpha \phi_{p}^{+}=0$ with $\alpha(k)=\beta(k) \cdot c(k)$. So if $c(k) \neq 0$ then there is always a non-zero multiple $\phi$ of $\phi_{p}^{+}$which satisfies $(B-\Theta) \phi=0$. Again, turning $k$ to $-k$ leaves $\phi_{p}^{+}$invariant, and we arrive at a contradiction if we take $B=B_{\infty}^{+}$. So $c(k) \equiv 0$.

However, from (3.12) ${ }^{-}$we see that $d_{2 j}^{-}$for $j<l$ is a polynomial in $\lambda$ of degree $j$, with a positive coefficient in front of $\lambda^{j}$. Considering the term in (3.17) with $s=0$ we get that, if $l \in \mathbb{Z}, c(\lambda)$ is a polynomial in $\lambda$ of degree $l$, with a non-zero coefficient in front of $\lambda^{l}$. So $c \equiv 0$ implies that $l=\frac{1}{2}+v_{p}, v_{p} \in \mathbb{Z}_{>0}$, excluding the possibility that the eigenfunctions are double valued around $p$. 
Remark. The last argument also shows that no non-zero multiple $\phi$ of the recessive solution at $p$ satisfies an equation of the form $(B-\Theta) \phi=0$.

Proposition 3.3. Let $V$ be an arbitrary meromorphic function in a neighborhood of $p \in \mathbb{C}$, with Laurent expansion

$$
V(x)=\sum_{r \geqq-2} c_{r} \cdot(x-p)^{r} .
$$

Then all eigenfunctions of $L=-\partial_{x}^{2}+V(x)$ are single-valued around $p$ if and only if

$$
c_{-2}=v_{p}\left(v_{p}+1\right) \quad \text { for some } v_{p} \in \mathbb{Z}_{>0}
$$

and

$$
c_{2 j-1}=0 \quad \text { for all integers } j \text { such that } 0 \leqq j \leqq v_{p} .
$$

Finally, if this is the case, then every eigenfunction $\phi$ has a Laurent expansion of the form

$$
\phi(x)=(x-p)^{-v_{p}} \cdot \sum_{r=0}^{\infty} d_{r} \cdot(x-p)^{r},
$$

with

$$
d_{2 j-1}=0 \quad \text { for all integers } j \text { such that } 1 \leqq j \leqq v_{p} .
$$

Proof. The proof of Lemma 3.2 has already shown us that $l=\frac{1}{2}+v_{p}$ for some $v_{p} \in \mathbb{Z}_{>0}$, so $c_{-2}=l^{2}-\frac{1}{4}=v_{p}\left(v_{p}+1\right)$. Also that $d_{2 j}^{-}$, for $0 \leqq j \leqq v_{p}$, is a polynomial in $\lambda$ of degree $j$, with positive leading coefficient.

Let $j_{0}$ be the smallest integer $j$ such that $0 \leqq j \leqq v_{p}$ and $c_{2 j-1} \neq 0$. On the righthand side of $(3.12)^{-}$for $r=2 j-1$ there appear only terms with $s$ odd $\geqq 2 j_{0}-1$, so $r-2-s$ even $\leqq 2\left(j-j_{0}-1\right)$, or with $s$ even $\geqq 0, r-s-2$ odd $\leqq 2 j-3$. By introduction over $j$ it follows that $d_{2 j-1}^{-}=0$ for $1 \leqq j \leqq j_{0}$ and that $d_{2 j-1}^{-}$is a polynomial in $\lambda$ of degree $\leqq j-j_{0}-1$ for $j_{0}<j \leqq v_{p}$. The only terms containing $\lambda^{j-j_{0}-1}$ are $c_{2 j_{0}-1} \cdot d_{2\left(j-j_{0}-1\right)}^{-}$and $\left(c_{0}-\lambda\right) \cdot d_{2 j-3}^{-}$. It follows, again by induction over $j$, that the leading coefficient in $d_{2 j-1}^{-}$is equal to $c_{2 j_{0}-1}$ times a strictly negative number, using that the coefficient in front of the left-hand side of $(3.12)^{-}$is negative if $r=2 j-1,1 \leqq j \leqq v_{p}$.

Now the right-hand side of (3.17) for $l=\frac{1}{2}+v_{p}$ contains terms for $s$ odd $\geqq 2 j_{0}-1$, the leading one being $c_{2 j_{0}-1} \cdot d_{2\left(v_{p}-j_{0}\right)}^{-}$, and for $s$ even $\geqq 0$, for which the leading one is $\left(c_{0}-\lambda\right) \cdot d_{2 v_{p}-1}^{-}$. We see that $c$ is a polynomial of degree $v_{p}-j_{0}$ in $\lambda$, with a leading coefficient equal to a positive multiple of $c_{2 j_{0}-1}$. If $j_{0}=v_{p}, d_{2 v_{p}-1}^{-}=0$, but still $c$ is a non-zero constant. The conclusion is that $c \equiv 0$ implies (3.21), which in turn implies (3.23).

If finally (3.21), and thus (3.23), holds, then on the right-hand side of (3.17) for $l=\frac{1}{2}+v_{p}$, either $s$ is odd $\leqq 2 v_{p}-1$, or $2 l-2-s$ is odd $\leqq 2 v_{p}-1$. In both cases $\check{c}_{s} \cdot d_{2 l-2-s}^{-}=0$, so $c \equiv 0$.

Theorem 3.4. Let $V$ be a rational function with $V(\infty)=0$. Then the following properties are equivalent.

a) All eigenfunctions of $-\partial_{x}^{2}+V(x)$ are meromorphic in $\mathbb{C}$.

b) All eigenfunctions of $-\partial_{x}^{2}+V(x)$ are of the form $e^{i k x} \cdot a^{+}(x, k)$ $+e^{-i k x} a^{-}(x, k)$ with $x \mapsto a^{ \pm}(x, k)$ rational and bounded at infinity.

c) At each pole $p$ of $V$, (3.19)-(3.21) hold.

d) $V$ is obtained from $V=0$ by finitely many rational Darboux transformations.

e) The potentials in the $K d V$-flow starting at $V$ remain rational. 
f) $V$ is obtained from $\frac{(v+1) v}{x^{2}}$, with $v$ an integer $\geqq 0$, by applying the flows in the
$K d V$ hierarchy. Proof. a) $\Leftrightarrow$ c) is Proposition 3.3. If a) holds then the analytic continuation of $x \mapsto e^{-( \pm i k x)} \phi_{\infty}^{ \pm}(x, k)$ to $\widetilde{S}_{\infty}^{ \pm}(k)$ in $(2.8)$ is bounded at $\infty$. Because it is single-valued it follows that $\phi_{\infty}^{ \pm}(x, k)=e^{ \pm i k x} \cdot a^{ \pm}(x, k)$ with $x \mapsto a^{ \pm}(x, k)$ rational and bounded at $\infty$. This proves a) $\Leftrightarrow$ b). d) $\Rightarrow$ a) follows from the description of $\operatorname{Ker}(\tilde{L}-\lambda)$ as the $Q$-image of $\operatorname{Ker}(L-\lambda)$, if $\widetilde{L}$ is obtained from $L$ by a rational Darboux transfor-

mation. See $(0.4)-(0.7)$. We now prove a) $\Rightarrow \mathrm{d})$.
Because $x=\infty$ is a regular singular point for $\left(-\partial_{x}^{2}+V\right) \phi=0[$ use that $V(x)$ $=O\left(\frac{1}{x^{2}}\right)$ because of $\left.\left.\mathrm{c}\right)\right]$, the solutions $\phi$, which are meromorphic in $\mathbb{C}$, now also have finite order at $\infty$, so they are rational. Using their single-valuedness it follows that

$$
V(x) \sim \frac{v(v+1)}{x^{2}} \text { as } x \rightarrow \infty, \text { for some } v \in \mathbb{Z}_{\geqq 0} .
$$

Comparison with (3.19) shows that

$$
v(v+1)=\sum_{\substack{p \in \mathbb{C} \\ p \text { pole of } V}} v_{p}\left(v_{p}+1\right) .
$$

Now let $\phi_{0} \in \operatorname{Ker}\left(-\partial_{x}^{2}+V\right)$ be recessive at $\infty$, that is, $\phi_{0}(x) \sim x^{-v}$ for $x \rightarrow \infty$. Then $-2\left(\frac{\phi_{0}^{\prime}}{\phi_{0}}\right)^{\prime} \sim-2\left(\frac{-v}{x}\right)^{\prime}=-\frac{2 v}{x^{2}}$ as $x \rightarrow \infty$. So the potential

$$
\tilde{V}(x)=V(x)-2\left(\frac{\phi_{0}^{\prime}(x)}{\phi_{0}(x)}\right)^{\prime}
$$

obtained from $V$ by means of the Darboux transformation with the solution $\phi_{0}$, will satisfy

$$
\tilde{V}(x) \sim \frac{\tilde{v}(\tilde{v}+1)}{x^{2}} \text { as } x \rightarrow \infty,
$$

with $\tilde{v}(\tilde{v}+1)=v(v+1)-2 v=(v-1) v$, that is,

$$
\tilde{v}=v-1 \text {. }
$$

Because $\tilde{V}$ is rational, $\tilde{V}(\infty)=0$, and $\tilde{V}$ satisfies a) again, the procedure can be repeated, and after $v$ Darboux transformations with eigenfunctions for the eigenvalue 0 which are recessive at $\infty$, we end up with a potential such that $v=0$. However, because $v_{p}\left(v_{p}+1\right)>0$ for each pole $p$ of $V$ on the right-hand side of (3.25), it follows that now $V$ has no poles anymore: it is a polynomial and because $V(\infty)=0, V=0$.

Because in the above notation, $\tilde{\phi}_{0}=1 / \phi_{0}$ is an eigenfunction for $-\partial_{x}^{2}+\tilde{V}$ for the eigenvalue 0 and the Darboux transformation applied to $\widetilde{V}$ with $\tilde{\phi}_{0}$ gives $V$ back, we see that $V$ is obtained from $V=0$ by applying $v$ Darboux transformations, each time using an eigenfunction for the eigenvalue 0 which is dominant at $\infty$.

Finally, the equivalence among d), e), and f) is one of the main results in Adler and Moser [2] and Airault, McKean, Moser [3]. 
A different proof can be obtained from Segal and Wilson [23], starting from the simple behavior of the eigenfunctions as a function of $k$ (rather than of $x$ ). In fact, we have:

$$
\phi_{\infty}^{ \pm}(x, k)=( \pm i k)^{-v} \cdot\left(\partial_{x}-v_{v}(x)\right) \circ \ldots \circ\left(\partial_{x}-v_{1}(x)\right) e^{ \pm i k x}
$$

with $v_{1}(x), \ldots, v_{v}(x)$ rational functions of $x$. This follows from the description of the new eigenfunctions after a Darboux transformation, preceding Theorem 0.1 . In particular, the amplitudes $a_{\infty}^{ \pm}(x, k)=\phi_{\infty}^{ \pm}(x, k) / e^{ \pm i k x}$ are polynomials in $k^{-1}$ of degree $\leqq v$.

Remarks. Because of the property f), we got into the habit of calling the potentials $V$ described in Theorem 3.4 as the rational $K d V$ potentials and the number $v$ in (3.24) could be called the order of $V$.

Now let $V$ be such a potential, $\phi_{0}$ an arbitrary non-zero eigenfunction of $-\partial_{x}^{2}+V$ for the eigenvalue 0 and $\tilde{V}$ as in (3.26). Then $\tilde{V}(x) \sim \frac{\tilde{v}(\tilde{v}+1)}{x^{2}}$ as $x \rightarrow \infty$ with $\tilde{v}=v+1$ if $\phi_{0}$ is dominant at $x=\infty$ and $\tilde{v}=v-1$ if $\phi_{0}$ is recessive at $x=\infty$. A special case occurs for $v=0$, because then the Darboux transformation leaves $V=0$ invariant if we take $\phi_{0}$ recessive at $\infty$. For the general $\phi_{0}$, the parameter in $\tilde{V}$ represents a translation. Letting $\phi_{0}$ approximate the solution which is recessive at $\infty$, the translation parameter goes to $\infty$ and $\widetilde{V}$ converges to 0 . Later Darboux transformations transfer this to the statement that every potential of order $v-1$ can be approximated by potentials of order $v$, which will be denoted by $v \rightarrow v-1$. See Sect. 7.7 for an alternative proof. Denoting the relation of being related by a Darboux transformation by a dashed double arrow $\leftrightarrow$, we get the following diagram

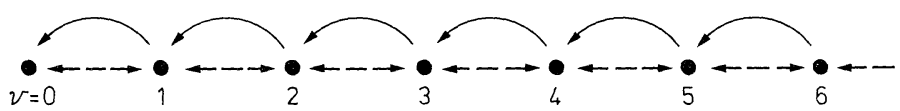

\section{Diagram 3-1}

The number $v$ here is also equal to the number of parameters in the family of potentials of order $v$. For refinements of this diagram (see Sect. 7.7).

If $\tilde{V}$ is obtained from $V$ as in (3.26), define the $\tilde{v}_{\tilde{p}}, \tilde{p}$ a pole of $\tilde{V}$ in the same way as the $v_{p}$ are defined for $V$. New poles of $\tilde{V}$, that is, poles $\tilde{p}$ which are regular points of $V$, appear at the zeros of $\phi_{0}$. These zeros are simple: a solution $\phi$ of a second order differential equation such that $\phi(x)=\phi^{\prime}(x)=0$ at a regular point $x$ is identically zero. It follows that $\tilde{v}_{\tilde{p}}=1$ at each new pole $\tilde{p}$. If $\tilde{p}$ is also a pole of $V$, then $\tilde{v}_{\tilde{p}}=v_{p}+1$ if $\phi_{0}$ is recessive at $p$, and $\tilde{v}_{\tilde{p}}=v_{p}-1$ otherwise. Because the generic element $\phi_{0}$ of $\operatorname{Ker}\left(-\partial_{x}^{2}+V\right)$ is not recessive at any pole $p$ of $V$, we see that after the generic sequence of rational Darboux transformations, $v_{p}=1$ for all poles $p$, and with each transformation, all poles of $V$ are eliminated and replaced by new ones at the zeros of $\phi_{0}$. So among the rational $\mathrm{KdV}$ potentials of order $v$, those of the form

$$
V(x)=\sum_{p \in \mathscr{P}} \frac{2}{(x-p)^{2}}, \quad \mathscr{P} \text { a subset of } \mathbb{C} \text { with } \frac{1}{2} v(v+1) \text { elements },
$$

form an open dense subset. The conditions (3.19)-(3.21) now read

$$
\sum_{\substack{q \in \mathscr{P} \\ q \neq p}} \frac{1}{(p-q)^{3}}=0 \text { for all } p \in \mathscr{P} \text {. }
$$


In Airault-McKean-Moser [3] the set of configurations $\mathscr{P}$ of points $p$ in $\mathbb{C}$ satisfying (3.30) is called the locus. It is proved there that the closure (in the space of rational functions) of the set of potentials $V$ in (3.29) with $\mathscr{P}$ in the locus, is equal to the set of potentials satisfying e) in Theorem 3.4. It is also shown there that for each $V$ in e), after flowing it out with $\mathrm{KdV}$, it immediately becomes of the form (3.29), with $\mathscr{P}$ in the locus. This proves that the latter potentials form a dense subset in a quite different way.

Note that (3.19)-(3.21) means that in general

$$
V(x)=\sum_{p \in \mathscr{P}} \frac{v_{p}\left(v_{p}+1\right)}{(x-p)^{2}}
$$

with $\mathscr{P}$ a finite subset of $\mathbb{C}$, and $v_{p} \in \mathbb{Z}_{>0}$ for $p \in \mathscr{P}$ being such that

$$
\sum_{\substack{q \in \mathscr{P} \\ q \neq p}} \frac{v_{q}\left(v_{q}+1\right)}{(q-p)^{2 j+1}}=0 \text { for } 1 \leqq j \leqq v_{p} \text { and each } p \in \mathscr{P} .
$$

This gives a description of the closure of the locus, implicitly asked for in [3].

In Adler and Moser [2], the rational $\mathrm{KdV}$ potentials of order $v$ are described as

$$
V(x)=-2\left(\frac{\theta^{\prime}(x)}{\theta(x)}\right)^{\prime}
$$

where $\theta(x)=\theta_{v}(x)$ is a polynomial defined by a recursive formula in $v$, cf. (7.1). If $V$ is as in (3.32), then we can take

$$
\theta(x)=\prod_{p}(x-p)^{\frac{1}{2} v_{p}\left(v_{p}+1\right)} .
$$

In Sect. 7.1 another description of $\theta(x)$ found by Sato will be used.

Theorem 3.5. Let $V$ satisfy any of the equivalent conditions a)-f) in Theorem 3.4. The eigenfunction $\phi_{\infty}^{ \pm}(x, k)$ in (2.4) satisfies an equation of the form $B^{ \pm}\left(k, \partial_{k}\right) \phi_{\infty}^{ \pm}(x, k)$ $=\Theta(x) \phi_{\infty}^{ \pm}(x, k)$ if and only if the polynomial $\Theta$ has the property that

$$
\Theta^{(2 j-1)}(p)=0 \text { for all } 1 \leqq j \leqq v_{p} \text {, for each pole } p \in \mathbb{C} \text { of } V .
$$

Proof. From Proposition 3.3 we know that

$$
\partial_{x}^{2 j-1}\left[(x-p)^{v_{p}} \cdot \phi_{\infty}^{ \pm}(x, k)\right]_{x=p}=0 \text { for } 1 \leqq j \leqq v_{p} .
$$

This remains true if $\phi_{\infty}^{ \pm}$is replaced by $B^{ \pm} \phi_{\infty}^{ \pm}$, by applying $B\left(k, \partial_{k}\right)$ to (3.35). So, if $B^{ \pm} \phi_{\infty}^{ \pm}=\Theta \phi_{\infty}^{ \pm}$, then also

$$
\partial_{x}^{2 j-1}\left[(x-p)^{v_{p}} \cdot \Theta(x) \cdot \phi_{\infty}^{ \pm}(x, k)\right]_{x=0}=0 \quad \text { for } \quad 1 \leqq j \leqq v_{p} .
$$

Expanding

$$
(x-p)^{v_{p}} \cdot \phi_{\infty}^{ \pm}(x, k)=\sum_{s=0}^{v_{p}} \frac{1}{(2 s) !} \delta_{2 s} \cdot(x-p)^{2 s}+O\left((x-p)^{2 v_{p}+1}\right),
$$

(3.36) can be written as

$$
\sum_{s=0}^{j=1}\left(\begin{array}{c}
2 j-1 \\
2 s
\end{array}\right) \cdot \Theta^{(2 j-1-2 s)}(p) \cdot \delta_{2 s}=0, \quad 1 \leqq j \leqq v_{p} .
$$


In view of the remark following Lemma $3.2, \delta_{0} \neq 0$ and (3.34) now follows by induction on $j$.

Conversely, now assume that (3.34) holds, which implies (3.36) in view of (3.38).

Let $B^{ \pm}=B^{ \pm}\left(k, \partial_{k}\right)$ be the unique differential operator satisfying (2.22) for $q=m, m-1, \ldots, 0$. In view of b) of Theorem 3.4, this means that

$$
\left(B^{ \pm}\left(k, \partial_{k}\right)-\Theta(x)\right) \phi_{\infty}^{ \pm}(x, k)=e^{ \pm i k x} R^{ \pm}(x, k),
$$$$
\text { with } x \mapsto R^{ \pm}(x, k) \text { rational and } R^{ \pm}(\infty, k)=0 \text {. }
$$

Because $\phi_{\infty}^{ \pm}(x, k)$, and therefore also $R_{\infty}^{ \pm}(x, k)$, only has poles at $p$ of order $\leqq v_{p}$, we can write

$$
R^{ \pm}(x, k)=\sum_{p \in \mathscr{P}} \sum_{r=0}^{v_{p}-1} R_{p, r}^{ \pm}(k) \cdot(x-p)^{r-v_{p}} .
$$

Expanding $e^{ \pm i k x}$ at $p$, we get that the coefficient of $(x-p)^{2 j-1-v_{p}}$ in the expansion of $e^{ \pm i k x} R^{ \pm}(x, k)$ at $p$ is equal to $e^{ \pm i k p}$ times

$$
\begin{aligned}
& \sum_{r=0}^{2 j-1} \frac{( \pm i k)^{2 j-1-r}}{(2 j-1-r) !} R_{p, r}^{ \pm}(k) \quad \text { if } \quad 2 j-1<v_{p}, \\
& \sum_{r=0}^{v_{p}-1} \frac{( \pm i k)^{2 j-1-r}}{(2 j-1-r) !} R_{p, r}^{ \pm}(k)+O\left(( \pm i k)^{2 j-1-v_{p}}\right) \text { times } R_{q, s}^{ \pm}(k), \quad q \neq p \\
& \text { if } 2 j-1 \geqq v_{p} .
\end{aligned}
$$

Now (3.35), (3.36), (3.39) imply that the expressions in (3.41) have to be equal to 0 for all integers $j$ such that $1 \leqq j \leqq v_{p}$.

Dividing the equations by $( \pm i k)^{2 j-v_{p}}$ and using

$$
( \pm i k)^{v_{p}-1-r} R_{p, r}^{ \pm}=S_{p, r}^{ \pm}
$$

as the new unknowns (notice that $v_{p}-1-r \geqq 0$, so $R_{p, r}^{ \pm}$is a non-positive power of $\pm i k$ times $S_{p, r}^{ \pm}$), we get

$$
\begin{aligned}
& \sum_{r=0}^{2 j-1} \frac{1}{(2 j-1-r) !} S_{p, r}^{ \pm}=0 \quad \text { if } \quad 2 j<v_{p}, \quad j \geqq 1, \\
& \sum_{r=0}^{v_{p}-1} \frac{1}{(2 j-1-r) !} S_{p, r}^{ \pm}+O\left(k^{-1}\right) \quad \text { times the } \quad S_{q, s}^{ \pm}, \quad q \neq p, \\
& \text { if } 2 j-1 \geqq v_{p}, \quad j \leqq v_{p} .
\end{aligned}
$$

Consider the $v_{p} \times v_{p}$ matrix

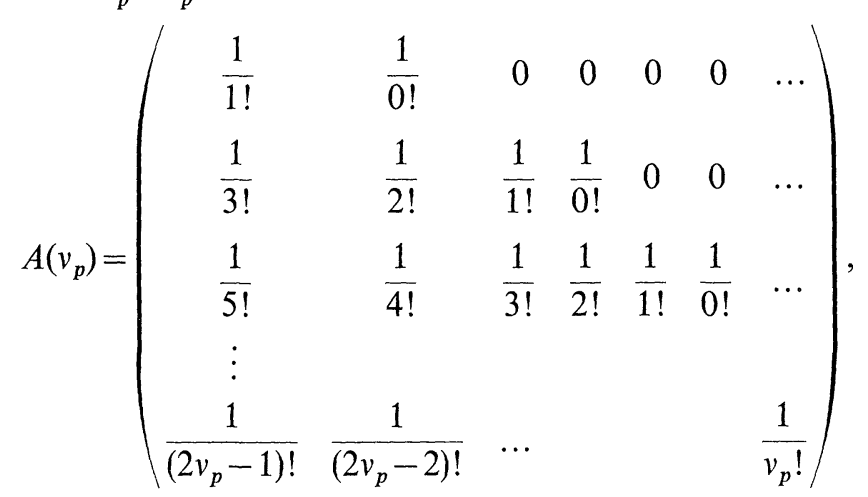


and let $A$ be the $\left(\sum v_{p}\right) \times\left(\sum v_{p}\right)$-matrix obtained by putting the $A\left(v_{p}\right)$ as blocks along the diagonal, the rest being zero. If we write $S^{ \pm}$for the vector of all $S_{p, r}^{ \pm}$, then (3.43) is an equation of the form

$$
\left(A+B^{ \pm}(k)\right) S^{ \pm}=0, \quad B^{ \pm}(k)=O\left(\frac{1}{k}\right) \quad \text { as } \quad k \rightarrow \infty .
$$

So, if $A$ is invertible, then $A+B^{ \pm}(k)$ is invertible for large $|k|$ and we conclude that $S^{ \pm}=0$, implying that $\left(B^{ \pm}\left(k, \partial_{k}\right)-\Theta(x)\right) \phi_{\infty}^{ \pm}(x, k)=0$ for large $|k|$; but then for all $k$ because of the analytic dependence on $k$.

$A$ is invertible if and only if $A\left(v_{p}\right)$ in (3.44) is invertible for each $p$. On the matrix for $v_{p}=\infty$ we perform the following column operations. Remove the last non-zero entry of each row, which is to the right of the diagonal element, by subtracting a suitable multiple of the column immediately to the left of it. Repeating this procedure one ends up with a lower triangular matrix, with $1, \frac{1}{3}, \frac{1}{3 \cdot 5}, \frac{1}{3 \cdot 5 \cdot 7}$, etc., on the diagonal. So,

$$
\operatorname{det} A\left(v_{p}\right)=1^{-v_{p}} \cdot 3^{1-v_{p}} \cdot 5^{2-v_{p}} \cdot \ldots \cdot(2 j-1)^{j-1-v_{p}} \cdot \ldots \cdot\left(2 v_{p}-1\right)^{-1}>0 .
$$

This completes the proof of Theorem 3.5.

Remark. $\operatorname{det} A(v)>0$, for all $v \geqq 1$, are the Hurwitz inequalities for the coefficients of $e^{x}=\sum_{k=0}^{\infty} \frac{1}{k !} x^{k}$ which, if $e^{x}$ would be a polynomial, would express that all its zeros have negative real part. These inequalities would follow from Hurewitz's theorem [15] if, for each $n$, all zeros of $\sum_{k=0}^{n} \frac{1}{k !} x^{k}$ have negative real part. This, however, is already false for $n=5$. Asymptotically for $n \rightarrow \infty$, about $\left(\frac{1}{2}-\frac{1}{\pi \cdot e} n\right.$ of the zeros have positive real part, cf. Szegö [25].

The space $\mathscr{B}=\mathscr{B}_{V}$ of polynomials $\Theta$ such that $(\operatorname{ad} L)^{m+1}(\Theta)=0$ for some $m \geqq 0$ form an algebra. For the proof, use that $(\operatorname{ad} L)(P \cdot Q)=(\operatorname{ad} L)(P) \cdot Q+P \cdot(\operatorname{ad} L)(Q)$. If $L=-\partial_{x}^{2}+V$ with $V(\infty)$ finite, then this can be phrased as follows: the space $\mathscr{B}^{ \pm}$ of operators $B^{ \pm}=B^{ \pm}\left(k, \partial_{k}\right)$ such that $B^{ \pm}\left(k, \partial_{k}\right) \phi_{\infty}^{ \pm}(x, k)=\Theta(x) \phi_{\infty}^{ \pm}(x, k)$ for some $\Theta=\Theta(x)$ obviously forms an algebra, the mapping $B^{ \pm} \rightarrow \Theta$ is a homomorphism: $\mathscr{B}^{ \pm} \rightarrow \mathscr{B}$, which, as we have seen, is bijective, so the algebras $\mathscr{B}^{ \pm}$and $\mathscr{B}$ are isomorphic. In particular, $\mathscr{B}^{ \pm}$is commutative. More precisely:

Proposition 3.6. If $B_{0} \in \mathscr{B}^{ \pm}$has positive degree, then $\mathscr{B}^{ \pm}$is equal to the space of all operators $B=B\left(k, \partial_{k}\right)$ which commute with $B_{0}$.

Proof. We have $B_{0} \phi_{\infty}^{ \pm}=\Theta_{0}(x) \cdot \phi_{\infty}^{ \pm}$with $\operatorname{deg} \Theta_{0}>0$. From the asymptotics for $x, k \rightarrow \infty$ we see that the large eigenvalues $t$ of $B_{0}$ are simple. But $B$ leaves $\operatorname{Ker}\left(B_{0}-\Theta_{0}(x)\right)$ invariant, so $B \phi_{\infty}^{ \pm}(x, \cdot)=\Theta(x) \cdot \phi_{\infty}^{ \pm}(x, \cdot)$ for some $\Theta(x)$, for large $x$. But this proves that $B \in \mathscr{B}^{ \pm}$.

It has been proved by Schur [22], using fractional powers of ordinary differential operators as in Gelfand and Dikii [9], that in general the algebra of differential operators which commute with a given $B_{0}$ of positive degree is commutative. It is also finitely generated, cf. Giertz et al. [10]. 
If $V$ is a rational $\mathrm{KdV}$ potential, then the algebra $\mathscr{B}^{ \pm}$apparently is isomorphic to the algebra $\mathscr{B}_{V}$ of polynomials $\Theta$ satisfying (3.34). That these form an algebra can be checked directly.

Writing $m_{a}=\{f \in \mathbb{C}[x] ; f(a)=0\}, a \mapsto \mathscr{B}_{V} \cap m_{a}$ is a bijection from $\mathbb{C}$ onto the space of non-zero prime ideals in $\mathscr{B}_{V}$ exhibiting the latter as a rational curve $\mathscr{C}_{V}$. For any set of generators $\Theta_{1}, \ldots, \Theta_{n}$ of $\mathscr{B}_{V}$ the map $x \mapsto\left(\Theta_{1}(x), \ldots, \Theta_{n}(x)\right)$ represents $\mathscr{C}_{V}$ as an algebraic curve in $\mathbb{C}^{n}$. The curve $\mathscr{C}_{V}$ is singular exactly at the points coming from $\mathscr{P}$; it therefore seems that we are not quite in the framework of Krichever [17]. It is also not too hard to prove that $\mathscr{B}_{V}$ is isomorphic to $\mathscr{B}_{V^{\prime}}$ (as algebras) if and only if there are $a, b \in \mathbb{C}, a \neq 0$, such that $v_{a p+b}^{\prime}=v_{p}$ for all $p \in \mathscr{P}$.

With a modification of the proof in Hartshorne [14, p. 310], one can show that the minimal number of generators is $\leqq 3$.

In the most degenerate case $V(x)=\frac{v(v+1)}{x^{2}}, v \in \mathbb{Z}_{>0}, \mathscr{B}_{V}$ has two generators $\left[\Theta(x)=x^{2}\right.$ and $\left.\Theta(x)=x^{2 v+1}\right]$ in contrast with all other Bessel cases where $\mathscr{B}_{V}$ has only one generator. (See the last remarks at the end of Sect. 5.)

Let $V$ be a rational $\mathrm{KdV}$ potential of order $v$. As discussed in the remarks following Theorem $3.4, V$ can be approximated by rational $\mathrm{KdV}$ potentials $\widetilde{V}$ of the same order but such that $v_{\tilde{p}}=1$ for all poles $\tilde{p}$ of $\tilde{V}$. In the limit $\frac{1}{2} v_{p}\left(v_{p}+1\right)$ of the poles $\tilde{p}$ of $\tilde{V}$ come together at the pole $p$ of $V$. Now

$$
\mathscr{B}_{\tilde{V}}=\left\{\Theta ; \Theta^{\prime}(\tilde{p})=0 \text { for all poles } \tilde{p} \text { of } \tilde{V}\right\},
$$

and a "confluent Vandermonde matrix" argument yields that

$$
\lim _{\tilde{V} \rightarrow V} \mathscr{B}_{\tilde{V}}=\left\{\Theta ; \Theta^{(j)}(p)=0 \text { for } 1 \leqq j \leqq \frac{1}{2} v_{p}\left(v_{p}+1\right) \text { for all poles } p \text { of } V\right\} .
$$

A simple continuity argument shows that in general

$$
\lim _{\tilde{V} \rightarrow V} \mathscr{B}_{\tilde{V}} \subset \mathscr{B}_{V}
$$

so the inclusion is strict as soon as $v_{p}>1$ for some pole $p$ of $V$. In fact, the codimension is equal to $\sum_{p} \frac{1}{2} v_{p}\left(v_{p}-1\right)$.

In particular, the minimal degree of a nonconstant $\Theta$ in $\mathscr{B}_{V}$ is smaller than in $\mathscr{B}_{\tilde{V}}$, which means that if we search for $V$ as a solution to $(\operatorname{ad} L)^{m+1}(\Theta)=0$ with increasing $m$, then the "degenerate" $V$ will turn up earlier in the process. (In fact, the minimal degree is $\leqq \sum v_{p}+1$, but is not a function of the $v_{p}$ only, see Sect. 7.6.) In terms of the Adler-Moser polynomial $\theta$ in (3.33), the $\tilde{V}$ are characterized by the property that their $\tilde{\theta}$ have simple zeros. So in the space of rational $\mathrm{KdV}$ potentials $V$ of order $v$ the ones with $v_{p}>1$ for some $p$ form a hypersurface determined by a discriminant equation (=the complement of the "locus" of Airault, McKean, Moser [3]). This hypersurface usually has singularities, in particular, at the Bessel potential $\frac{v(v+1)}{x^{2}}$ from which they branch off. In terms of the equations $(\operatorname{ad} L)^{m+1}(\Theta)=0$ for increasing $m$, the effect is that at a certain stage singular families of $B$ appear which are then later "smoothed out" by embedding them in higher dimensional families. This is one of the confusing phenomena if one tries to solve $(\operatorname{ad} L)^{m+1}(\Theta)=0$ for increasing values of $m$, which also occurs in the "even case." 
In Sect. 4 it will be proved that if $V$ is a rational $\mathrm{KdV}$ potential and if there is a differential operator $B\left(k, \partial_{k}\right)$ of positive order and a $\Theta(x)$ such that $\operatorname{Ker}\left(-\partial_{x}^{2}+V(x)-\lambda\right) \cap \operatorname{Ker}\left(B\left(k, \partial_{k}\right)-\Theta(x)\right)$ is two-dimensional, then $V(x)$ $=\frac{v(v+1)}{x^{2}}$, the Bessel potential. So the rational KdV potentials, except the Bessel ones among them, are precisely the potentials $V$ such that there is a onedimensional (but no two-dimensional) common eigenspace. [In Sect. 5 it will also be proved that if $V(\infty)=\infty$, then $V$ is the Airy potential with a two-dimensional common eigenspace.]

\section{The Even Case}

Again, let $L=-\partial_{x}^{2}+V(x)$ with $V(\infty)=0$ and $\Theta=\Theta(x)$ with $\operatorname{deg} \Theta=m$ satisfying $(\operatorname{ad} L)^{m+1}(\Theta)=0$, but this time we assume that there is a function $c=c(k)$ such that the operators $B^{ \pm}$of order $m$ in (2.18) satisfy

$$
B^{-}\left(k, \partial_{k}\right)=c(k)^{-1} \circ B^{+}\left(k, \partial_{k}\right) \circ c(k) .
$$

Lemma 4.1. After a suitable translation in the $x$-variable, $\Theta$ and $V$ are even functions of $x, B^{+}=B^{-}$and $c$ is a constant. Conversely, if $V$ is an even function and $m$ is even, then there is an even $\underline{\Theta}$ with $\operatorname{deg} \Theta=m$ such that $(\operatorname{ad} L)^{m+1}(\underline{\Theta})=0$ and the corresponding operators $B^{ \pm}$satisfy $B^{+}=B^{-}$.

Proof. By induction over $p$ one gets that the polynomials $a_{p}^{ \pm}(k)$ in $\frac{1}{k}$, defined recurrently by $(2.6)$ with $a_{0}^{ \pm}(k) \equiv 1$, satisfy

$$
a_{p}^{-}(k)=a_{p}^{+}(-k) \text {. }
$$

It follows that $\partial_{k}^{s} a_{p}^{-}(k)=(-1)^{s}\left(\partial_{k}^{s} a_{p}^{+}\right)(-k)$, so from Eqs. (2.22) for $q=m, m-1, \ldots, 0$, which determine the coefficients $B_{j}^{ \pm}(k)$ of $B^{ \pm}\left(k, \partial_{k}\right)$, we get

$$
B_{j}^{-}(k)=(-1)^{j} B_{j}^{+}(-k) .
$$

Using that $\partial_{k}^{s} \circ c=\sum_{r=0}^{s}\left(\begin{array}{l}s \\ r\end{array}\right) \cdot\left(\partial_{k}^{r} c\right) \circ \partial_{k}^{s-r},(4.1)$ and (4.3) give

$$
(-1)^{j} B_{j}^{+}(-k)=c(k)^{-1} \sum_{r=0}^{m-j} B_{j+r}^{+}(k) \cdot\left(\begin{array}{c}
j+r \\
r
\end{array}\right) \cdot \partial_{k}^{r} c(k) \text { for all } j .
$$

Reading this equation for $j=m$, we get

$$
(-1)^{m} B_{m}^{+}(-k)=B_{m}^{+}(k),
$$

which implies that $m$ is even because $B_{m}^{ \pm}(k)=( \pm i)^{-m} . \Theta_{m}$ is a constant, see (2.23). Reading (4.4) for $j=m-1$ :

$$
(-1)^{m-1} B_{m-1}^{+}(k)=B_{m-1}^{+}(k)+c(k)^{-1} \cdot i^{-m} \cdot \Theta_{m} \cdot m \cdot \partial_{k} c(k) .
$$

Now (2.22) for $q=m-1$, using that $B_{m}^{ \pm}(k) \cdot( \pm i)^{m}=\Theta_{m}$, yields that $B_{m-1}^{ \pm}(k)$ $=( \pm i)^{1-m} \cdot \Theta_{m-1}$ is a constant as well. Combining this with the evenness of $m,(4.6)$ now reads

$$
\partial_{k} c(k)=-\frac{2 i}{m} \cdot \frac{\Theta_{m-1}}{\Theta_{m}} \cdot c(k)
$$


This means that $c(k)$ depends exponentially on $k$. By a translation in the $x$-variable, we can arrange that

$$
\Theta_{m-1}=0,
$$

a normalization which immediately leads to

$$
\begin{gathered}
c \text { is a constant, } B^{-}\left(k, \partial_{k}\right)=B^{+}\left(k, \partial_{k}\right), \\
B_{j}^{+}(k)=(-1)^{j} B_{j}^{+}(-k) \text { for all } j=m, m-1, \ldots, 0 .
\end{gathered}
$$

In view of the notation $B_{p}^{+}(k)=\sum_{s=0}^{m-p} B_{p, s}^{+} \cdot k^{-s}[$ see (2.27)], this means that $B_{p, s}=0$ for $p+s$ odd, or

$$
\beta_{s}^{+}(x) \underset{\operatorname{def}}{=} \sum_{j} B_{j, s}^{+} \cdot(i x)^{j}=(-1)^{s} \cdot \beta_{s}^{+}(-x) .
$$

Because $\beta_{0}^{+}(x)=\Theta(x),(4.11)$ for $s=0$ just means

$$
\Theta \text { is an even function of } x: \Theta(x)=\Theta(-x) \text {. }
$$

Now the coefficients $\alpha_{l}^{+}(x)$ in the asymptotic expansion (2.32) for $\phi_{\infty}^{+}(x, k)$ as $k \rightarrow \infty$, were determined recurrently from the $\beta_{s}^{+}(x)$ by the formula (2.41). Inserting (4.11) one obtains by induction over $l$ :

$$
\alpha_{l}^{+}(x)=(-1)^{l} \cdot \alpha_{l}^{+}(-x) \text { for all } x .
$$

In view of $V(x)=2 i \cdot \partial_{x} \alpha_{1}^{+}(x)$ [see (2.34)], we have proved that $V$ is an even function of $x$ as well.

For the last statement, take $\Theta(x)=\frac{1}{2}(\Theta(x)+\Theta(-x)) . B^{+}=B^{-}$now follows because $a_{p}^{-}(k)=(-1)^{p} a_{p}^{+}(k)\left[\right.$ use (2.6)], so $a_{p}^{+}(-k)=(-1)^{p} a_{p}^{+}(k)$. Now (4.3) can be read off from (2.22).

Remark. Another aspect of (4.10) is that $B^{+}\left(k, \partial_{k}\right)$ actually is equal to a linear differential operator $A\left(\lambda, \partial_{\lambda}\right)$ in $\lambda=k^{2}$, with coefficients that are rational functions in $\lambda$, that is, have no square root behavior in $\lambda$. In order to see this, note that

$$
\partial_{k}^{j}=\sum_{i=0}^{[j / 2]} \alpha_{j, i} \cdot k^{j-2 i} \cdot \partial_{\lambda}^{j-i},
$$

where the $\alpha_{j, i}$ are universal coefficients, determined recurrently by

$$
\alpha_{j, i}=2 \cdot \alpha_{j-1, i}+(j+1-2 i) \cdot \alpha_{j-1, i-1}, \quad \alpha_{0,0}=1, \quad \alpha_{j,<0}=0 .
$$

Now we can write

$$
B^{+}\left(k, \partial_{k}\right)=\sum_{\substack{j, s \\ j+s \text { even }}} \sum_{i=0}^{[j / 2]} B_{j, s}^{+} \cdot \alpha_{j, i} \cdot k^{-s+j-2 i} \cdot \partial_{\lambda}^{j-i},
$$

which proves the statement because $-s+j-2 i$ is even where $j+s$ is even.

From now on we assume that $B^{+}=B^{-}(\equiv B)$.

From Corollary 2.2 we see that the local solutions of $\left(L-k^{2}\right) \phi=0$, $(B-\Theta(x)) \phi=0$ form a two-dimensional complex vector space. In the description following (2.25), $\mathscr{S}$ is a holomorphic two-dimensional vector bundle; the analytic continuation of solutions defines a flat holomorphic connection in $\mathscr{S}$. Since $\mathscr{S}$ is 
spanned by the analytic continuation of ${ }^{0} \phi_{\infty}^{+},{ }^{0} \phi_{\infty}^{-}$and these functions, for $k$ fixed, form a basis of solutions of $\left(L-k^{2}\right) \phi=0, \mathscr{P}_{(x, k)}$ can be parametrized by the vector

$$
v(x, k)=\left(\begin{array}{c}
\phi(x, k) \\
\partial_{x} \phi(x, k)
\end{array}\right) \in \mathbb{C}^{2} \quad\left(\phi \in \mathscr{P}_{(x, k)}\right),
$$

for which we have the first order linear differential equation

$$
\partial_{x} v(x, k)=P(x, k) \cdot v(x, k), \quad P(x, k)=\left(\begin{array}{cc}
0 & 1 \\
V(x)-k^{2} & 0
\end{array}\right) .
$$

Having a connection means that we also have a first order linear differential equation in the $k$-direction

$$
\partial_{k} v(x, k)=Q(x, k) \cdot v(x, k), \quad Q(x, k)=\left(\begin{array}{ll}
a(x, k) & b(x, k) \\
c(x, k) & d(x, k)
\end{array}\right),
$$

for a suitable $a, b, c, d$; the flatness condition, equivalent to the two-dimensionality of the common solution space of (4.18), (4.19), is expressed by the compatibility condition

$$
\partial_{k} P+P Q=\partial_{x} Q+Q P .
$$

Following the method of Flaschka and Newell [8, pp. 87, 88], we shall first prove that (4.19) holds with $a, b, c, d$ being rational functions of $x$. Defining the fundamental solution

$$
{ }^{0} \Phi_{\infty}(x, k)=\left(\begin{array}{cc}
{ }^{0} \phi_{\infty}^{+}(x, k) & { }^{0} \phi_{\infty}^{-}(x, k) \\
\partial_{x}{ }^{0} \phi_{\infty}^{+}(x, k) & \partial_{x}{ }^{0} \phi_{\infty}^{-}(x, k)
\end{array}\right)
$$

[see (2.9)], it is obvious that

$$
Q(x, k)=\partial_{k}{ }^{0} \Phi_{\infty}(x, k) \circ{ }^{0} \Phi_{\infty}(x, k)^{-1} \text { in the sector } S_{\infty}^{0}(k) .
$$

Because the operators $L\left(x, \partial_{x}\right)-k^{2}$ and $B\left(k, \partial_{k}\right)-\Theta(x)$ are single-valued for $(x, k) \in D$, meaning that $\mathscr{S}$ is a holomorphic vector bundle over $D$ (that is, there is no need to pass to some covering of $D$ ), it is a priori clear that $Q$ is single-valued on $D$. In particular, in (4.21) we could have also replaced ${ }^{0} \phi_{\infty}^{ \pm}$by ${ }^{\pi} \phi_{\infty}^{ \pm}$and gotten a representation like (4.22) in $S_{\infty}^{\pi}(k)$. We will now investigate the asymptotic behavior of the right-hand side of (4.22) for $x \rightarrow \infty$ in $S_{\infty}^{0}(k)$, respectively $S_{\infty}^{\pi}(k)$ (with ${ }^{0} \Phi_{\infty}$ replaced by $\left.{ }^{\pi} \Phi_{\infty}\right)$. Because $S_{\infty}^{0}(k) \cup S_{\infty}^{\pi}(k)$ is a full punctured neighborhood of $\infty$, this will then provide the asymptotic behavior of $Q(x, k)$ as $x \rightarrow \infty$.

Because the trace of $P$ in (4.18) is equal to zero, the determinant of ${ }^{0} \Phi_{\infty}(x, k)$ is constant as a function of $x$. Because ${ }^{0} \Phi_{\infty}^{ \pm}(x, k) \sim e^{ \pm i k x}, \partial_{x}{ }^{0} \phi_{\infty}^{ \pm}(x, k) \sim \pm i k \cdot e^{ \pm i k x}$ as $x \rightarrow \infty$ in $S_{\infty}^{0}(k)$, it follows that

$$
\operatorname{det}^{0} \Phi_{\infty}(x, k)=-2 i k .
$$

Simplifying the notation somewhat, we get

$$
Q(x, k)=\frac{1}{-2 i k} \cdot\left(\begin{array}{cc}
\partial_{k} \phi^{+} \cdot \partial_{x} \phi^{-}-\partial_{k} \phi^{-} \cdot \partial_{x} \phi^{+} & -\partial_{k} \phi^{+} \cdot \phi^{-}+\partial_{k} \phi^{-} \cdot \phi^{+} \\
\partial_{k} \partial_{x} \phi^{+} \cdot \partial_{x} \phi^{-}-\partial_{k} \partial_{x} \phi^{-} \cdot \partial_{x} \phi^{+} & -\partial_{k} \partial_{x} \phi^{+} \cdot \phi^{-}+\partial_{k} \partial_{x} \phi^{-} \cdot \phi^{+}
\end{array}\right) .
$$


In view of (2.6) for $p=1$ we get, for $x \rightarrow \infty$ in $S_{\infty}^{0}(k)$, respectively $S_{\infty}^{\pi}(k)$,

$$
\begin{aligned}
\phi^{ \pm} & =e^{ \pm i k x}\left[1-\frac{V_{2}}{ \pm 2 i k x}+O\left(\frac{1}{x^{2}}\right)\right], \\
\partial_{x} \phi^{ \pm} & =e^{ \pm i k x}\left[ \pm i k\left(1-\frac{V_{2}}{ \pm 2 i k x}\right)+O\left(\frac{1}{x^{2}}\right)\right], \\
\partial_{k} \phi^{ \pm} & =e^{ \pm i k x}\left[ \pm i x\left(1-\frac{V_{2}}{ \pm 2 i k x}\right)+O\left(\frac{1}{x}\right)\right], \\
\partial_{k} \partial_{x} \phi^{ \pm} & =e^{ \pm i k x}\left[-x k\left(1-\frac{V_{2}}{ \pm 2 i k x}\right) \pm i+O\left(\frac{1}{x}\right)\right],
\end{aligned}
$$

and inserting this into (4.24) it follows that

$$
Q(x, k)=\left(\begin{array}{cc}
0 & \frac{x}{k} \\
-x k & \frac{1}{k}
\end{array}\right)+O\left(\frac{1}{x}\right) \text { as } x \rightarrow \infty .
$$

Now let $p$ be an irregular singular point for $\left(-\partial_{x}^{2}+V(x)-k^{2}\right) \phi=0$, that is assume that $V$ satisfies (3.2). The same argument as in the proof of Lemma 3.1 shows that $\phi_{p, j}^{ \pm}$satisfy differential equations of the form $\left(B_{p, j}^{ \pm}\left(k, \partial_{k}\right)-\Theta(x)\right) \phi_{p, j}^{ \pm}=0$. Corollary 2.2 now gives the existence of functions $c_{p, j}^{ \pm}(k)$ and constants $t_{p, j,+}^{ \pm}, t_{p, j,-}^{ \pm}$, such that

$$
\tilde{\phi}_{p, j}^{ \pm} \underset{\text { def }}{=} c_{p, j}^{ \pm}(k) \cdot \phi_{p, j}^{ \pm}=t_{p, j,+}^{ \pm}{ }^{0} \phi_{\infty}^{+}+t_{p, j,-}^{ \pm}{ }^{0} \phi_{\infty}^{-} .
$$

On the larger sector

$$
S_{p, j}=\left\{x \in \mathbb{C} ;|x-p| \text { small, } \varepsilon+2 \pi j<\arg c_{\mu}^{1 / 2}+\left(1+\frac{\mu}{2}\right) \cdot \arg (x-p)<2 \pi-\varepsilon+2 \pi j\right\},
$$

the analytic continuations of $\phi_{p, j}^{ \pm}$still satisfy the asymptotic expansion (3.4). Now, writing

$$
\tilde{\Phi}_{p, j}=\left(\begin{array}{cc}
\widetilde{\phi}_{p, j}^{+} & \widetilde{\phi}_{p, j}^{-} \\
\partial_{x} \widetilde{\phi}_{p, j}^{+} & \partial_{x} \widetilde{\phi}_{p, j}^{--}
\end{array}\right)
$$

the relations (4.26) mean that

$$
{ }^{0} \Phi_{\infty}(x, k)=\tilde{\Phi}_{p, j}(x, k) \circ T,
$$

for a "transfer" matrix $T$ which is independent of $x$ and $k$. Hence $\partial_{k}{ }^{\circ} \Phi_{\infty}$ $=\partial_{k} \tilde{\Phi}_{p, j} \circ T$, and so

$$
Q(x, k)=\partial_{k}{ }^{0} \Phi_{\infty}{ }^{\circ}{ }^{0} \Phi_{\infty}^{-1}=\partial_{k} \tilde{\Phi}_{p, j}{ }^{\circ} \tilde{\Phi}_{p, j}^{-1},
$$

which is a matrix like (4.24), but with $\phi^{ \pm}$replaced by $\tilde{\phi}_{p, j}^{ \pm}$and some function of $k$ as a factor in front. Because the exponential factors from the asymptotic expansion 
(3.4) cancel each other, it follows immediately that $Q(x, k)=O\left(|x-p|^{\kappa}\right)$ for $x \rightarrow p$ in $S_{p, j}$, for some $\kappa \in \mathbb{R}$. Using that the union of $S_{p, j}$ cover a full punctured neighborhood of $p$, the conclusion is that $x \mapsto Q(x, k)$ is meromorphic at $p$. [Actually, $x \mapsto b(x, k)$ is regular at $p$, using that $\omega^{+}+\omega^{-}=-\mu / 2>0$.]

Because at a regular singular point $p$ all solutions $\phi$ (and therefore the analytic continuations of ${ }^{0} \phi_{\infty}^{ \pm}$, too) satisfy estimates of the form $\phi(x, k)=O\left(|x-p|^{\kappa}\right)$ as $x \rightarrow p$ in any sector, one concludes immediately that $x \mapsto Q(x, k)$ is meromorphic at the regular singular points as well. Summarizing, we have proved the rationality of $x \mapsto Q(x, k)$, announced after (4.20).

Next we analyze the compatibility condition of (4.20):

$$
\begin{aligned}
& \partial_{x} a=c-\left(V-k^{2}\right) b, \\
& \partial_{x} b=d-a, \\
& \partial_{x} c=-2 k+\left(V-k^{2}\right)(a-d), \\
& \partial_{x} d=\left(V-k^{2}\right) b-c .
\end{aligned}
$$

Summing the first and the last equation we see that $a+d$ does not depend on $x$. More precisely, because

$$
\operatorname{Tr} Q(x, k)=\partial_{k}\left(\operatorname{det}^{0} \Phi_{\infty}(x, k)\right) \cdot\left(\operatorname{det}^{0} \Phi_{\infty}(x, k)\right)^{-1}=\partial_{k}(-2 i k) \cdot(-2 i k)^{-1}=k^{-1},
$$

using (4.22), (4.23), we even have

$$
a+d=k^{-1} .
$$

Now (4.31) leads to $a=-\frac{1}{2} \partial_{x} b+\frac{1}{2} k^{-1}, d=\frac{1}{2} \partial_{x} b+\frac{1}{2} k^{-1}, c=\left(V-k^{2}\right) b-\frac{1}{2} \partial_{x}^{2} b$, showing that the whole matrix $Q(x, k)$ can be expressed in terms of the function $b(x, k)$, which in turn satisfies the differential equation

$$
-\frac{1}{2} \partial_{x}^{3} b+2\left(V-k^{2}\right) \cdot \partial_{x} b+\partial_{x} V \cdot b+2 k=0 .
$$

From this equation it is obvious that $x \mapsto b(x, k)$ can only have poles at the poles $p$ of $V$. Writing

$$
V(x) \sim c_{\mu} \cdot(x-p)^{\mu}, \quad b(x, k) \sim b_{p}(k) \cdot(x-p)^{\beta} \quad \text { for } \quad x \rightarrow p,
$$

with $\mu \leqq-2\left(\int V\right.$ is rational!), $\beta<0, c_{\mu} \neq 0, b_{p}(k) \neq 0$, the leading term on the lefthand side of (4.33) is

and

$$
(2 \beta+\mu) \cdot c_{\mu} \cdot b_{p}(k) \cdot(x-p)^{\mu+\beta-1} \quad \text { if } \mu<-2
$$

$$
b_{p}(k)\left(-\frac{1}{2} \beta(\beta-1)(\beta-2)+(2 \beta-2) c_{\mu}\right) \cdot(x-p)^{\beta-3} \text { if } \quad \mu=-2 .
$$

So the conclusion is that

$$
\mu=-2 \quad \text { and } \quad c_{\mu}=\frac{1}{4} \beta(\beta-2)=l^{2}-\frac{1}{4} \quad \text { with } \quad l=\frac{1}{2}(1-\beta)>\frac{1}{2} .
$$

In other words, $x \mapsto b(x, k)$ can only have poles at regular singular points $p$ for $\left(-\partial_{x}^{2}+V(x)-k^{2}\right) \phi=0$, the monodromy around which has an eigenvalue \pm 1 , of algebraic multiplicity 2 .

Furthermore, all eigenfunctions of $-\partial_{x}^{2}+V(x)$ are single-valued around the poles of $b$. The generic $\phi \in \operatorname{Ker}\left(L-k^{2}\right) \cap \operatorname{Ker}(B-\Theta(x))$ can be written as $\phi(x, k)$ 
$=\gamma(k) \cdot \tilde{\phi}_{p}^{-}(x, k)$, where $\gamma(k) \neq 0$ and $\tilde{\phi}_{p}^{-}(x, k)$ is as in (3.16). Now turning $x$ around $p$, $\phi(x, k)$ is changed into $\phi(x, k)+\gamma(k) \cdot c(k) \cdot 2 \pi i \cdot \phi_{p}^{+}(x, k)$. So if $c(k) \neq 0$ (which is always the case for large $|k|$ if $l \in \mathbb{Z})$, it follows that $\gamma \cdot c \cdot \phi_{p}^{+} \in \operatorname{Ker}\left(L-k^{2}\right)$ $\cap \operatorname{Ker}(B-\Theta(x))$ is linearly independent of $\phi$.

If we replace the common eigenfunctions ${ }^{0} \phi_{\infty}^{+}$, respectively ${ }^{0} \phi_{\infty}^{-}$in (4.21) by $\gamma \cdot c \cdot \phi_{p}^{+}$, respectively $\phi=\gamma \cdot \widetilde{\phi}_{p}^{-}$, we get a fundamental solution $\Phi_{p}(x, k),{ }^{0} \Phi_{\infty}(x, k)$ $=\Phi_{p}(x, k) \circ T$ for a transfer matrix $T$ which does not depend on $x$ and $k$, so that $Q(x, k)=\partial_{k} \Phi_{p}(x, k) \circ \Phi_{p}(x, k)^{-1}$, as in (4.30). Replacing the functions $\phi^{+}$, respectively $\phi^{-}$, in the upper right corner of (4.24) by $\gamma c \phi_{p}^{+}$, respectively $\gamma \cdot \tilde{\phi}_{p}^{-}$, we see immediately that $b(x, k)=O(|x-p| \cdot|\log (x-p)|)$, so $x \mapsto b(x, k)$ has no pole at $x=p$. That $l=\frac{1}{2}+v_{p}, v_{p} \in \mathbb{Z}_{>0}$ now follows in the same way as in the last part of the proof of Lemma 3.2.

Remark. A similar argument also says that at the poles $p$ of $x \mapsto b(x, k)$ there is no $\gamma(k) \neq 0$ such that $\gamma \cdot \phi_{p}^{+} \in \operatorname{Ker}\left(L-k^{2}\right) \cap \operatorname{Ker}(B-\Theta(x))$.

We now turn to the asymptotics as $x \rightarrow \infty$ for $b$. Using (4.25) we may write (using that $b$ and $V$ are rational):

$$
b(x, k)=\frac{x}{k}+\sum_{s \geqq 1} b_{s}(k) \cdot x^{-s}, \quad V(x)=\sum_{r \geqq 2} V_{r} \cdot x^{-r} .
$$

Substituting this in (4.33) and collecting the coefficients of $x^{-s-1}$ :

$$
2 k^{2} \cdot s \cdot b_{s}=\frac{s-1}{k} \cdot V_{s+1}+\sum_{\substack{r, t \\ r+t=s}} V_{r} \cdot b_{t} \cdot(r+2 t)-\frac{1}{2}(s-2)(s-1) s \cdot b_{s-2},
$$

which determines the $b_{s}$ recurrently. We read off that $b_{1}=0, b_{2}=\frac{1}{4 k^{3}} V_{3}=0$ because $V$ is even, $b_{3}=\frac{1}{3 k^{3}} V_{4}$, etc. By induction one gets that $b_{s}=0$ for $s$ even, $b_{s}(k)$ is an odd function of $k$ for $s$ odd, $b_{s}(k)=O\left(k^{-3}\right)$ for $s \geqq 3$. Now $b(x, k)-\frac{x}{k}=\frac{A(x, k)}{B(x)}$ where $A(\cdot, k)$ and $B$ are polynomials with $B$ independent of $k$ because the poles of $b$ are all poles of $V$, which do not depend on $k$. From $A(x, k)=B(x) \cdot \sum_{s \geqq 1} b_{s}(k) \cdot x^{-s}$, one sees that the coefficients of $A(\cdot, k)$ are linear expressions in only finitely many of the $b_{s}(k)$, so we may conclude that

$$
b(x, k)=\frac{x}{k}+O\left(k^{-3}\right) \quad \text { as } \quad k \rightarrow \infty .
$$

By induction we also get that $b_{s}$ is a polynomial in $\frac{1}{k}$ of degree $\leqq s$, and the same argument as above shows that $b$ is a polynomial in $\frac{1}{k}$.

But then the degrees of $b_{s}$ (as polynomials in $\frac{1}{k}$ ) cannot increase indefinitely. Because the coefficient $\beta_{s}$ of $k^{-s}$ in $b_{s}$ satisfies

$$
2 s \beta_{s}=\left(V_{2} \cdot(2+2(s-2))-\frac{1}{2}(s-2)(s-1) s\right) \cdot \beta_{s-2},
$$


we see that necessarily

$$
V_{2}=\frac{1}{4}(s-2) s \text { for some odd } s>1,
$$

unless $b_{s}=0$ for all $s$, that is,

$$
b(x, k)=\frac{x}{k}, \quad V(x)=\frac{c}{x^{2}}, \quad \text { the Bessel potential. }
$$

From now on let us exclude the Bessel case. From (4.39) it follows that the only zero of $x \mapsto b(x, k)$ which does not move as $k$ varies is $x=0$. Because $\partial_{x} b(x, k)$ $=\frac{1}{k}+O\left(k^{-3}\right)$ for $k \rightarrow \infty$, this is a simple zero. Because the poles of $V$ do not move with $k$, it follows that $V$ has, except at $x=0$, only poles at the poles of $b$. From (4.33) one then reads off that if $x=0$ is the pole for $V$, then it is a pole of order 2 :

$$
V(x) \sim \frac{c-2}{x^{2}} \text { for } x \rightarrow 0
$$

Now $\left(-\partial_{x}^{2}+V(x)\right) \phi=0$, the equation for eigenvalue 0 , has a regular singular point at $x=\infty$, and from (4.41) we see that the monodromy around $\infty$ (that is, going around a circle enclosing all the poles of $V$ ) has -1 as its eigenvalue. Because all the eigenfunctions (and therefore those for $k=0$, too) are single-valued around all poles $p \neq 0$ of $V$, the monodromy around 0 is the same. Necessarily therefore

$$
c_{-2}=l^{2}-\frac{1}{4} \text { for some } l \in \mathbb{Z}_{\geqq 0} .
$$

We can now summarize the conclusions in

Proposition 4.2. After a suitable translation in the $x$-variable either $V(x)=\frac{c}{x^{2}}$ (Bessel) or

$$
\begin{aligned}
V(x)= & \frac{l^{2}-\frac{1}{4}}{x^{2}}+\sum_{p \in \mathscr{P}} \frac{v_{p}\left(v_{p}+1\right)}{(x-p)^{2}}, \text { where } l \in \mathbb{Z}_{\geqq 0}, \quad v_{p} \in \mathbb{Z}_{>0}, \quad v_{-p}=v_{p} \\
& \text { and } \mathscr{P} \text { is a finite subset of } \mathbb{C} \backslash\{0\}, \text { symmetric around } 0 .
\end{aligned}
$$

Furthermore, all eigenfunctions of $-\partial_{x}^{2}+V(x)$ are single-valued around all the poles $p \neq 0$ of $V$. Given (4.45), this last property is equivalent to

$$
\frac{l^{2}-\frac{1}{4}}{p^{2 j+1}}+\sum_{q \in \mathscr{P}\{\{p\}} \frac{v_{q}\left(v_{q}+1\right)}{(p-q)^{2 j+1}}=0 \quad \text { for } \quad 1 \leqq j \leqq v_{p}, \text { all } p \in \mathscr{P} .
$$

Remark. The last argument in the proof of Lemma 3.2 gave that around $x=0$ the eigenfunction is a multiple of $\tilde{\phi}_{p}^{-}(x, k)[$ in (3.16)] with $p=0$, with $c(k)$ a polynomial in $\lambda=k^{2}$ of true degree $l$, so the monodromy around $x=0$ (and therefore also around $x=\infty)$ is of the form $\left(\begin{array}{rr}-1 & c(k) \\ 0 & -1\end{array}\right)$ on a suitable basis of eigenfunctions.

Proposition 4.3. If $V$ is a potential as in (4.45), (4.46), then it can be obtained from $\frac{(l+\mu)^{2}-\frac{1}{4}}{x^{2}}$ by $\mu$ Darboux transformations, using at each step an eigenfunction for the 
eigenvalue 0 which is dominant at $x=0$. The potentials obtained in this way form a smooth $\mu$-dimensional family having $\frac{(l+2 \mu)^{2}-\frac{1}{4}}{x^{2}}$ in its closure. Conversely, every potential obtained from $\frac{\mathscr{L}^{2}-\frac{1}{4}}{x^{2}}$ for some $\mathscr{L} \in \mathbb{Z}_{\geqq 0}$ by finitely many rational Darboux transformations, is of the form (4.45), (4.46).

Proof. Assume (4.45), (4.46). The equation $\left(-\partial_{x}^{2}+V(x)\right) \phi=0$ has a regular singular point at $x=\infty$. The monodromy around $\infty$ is the same as the monodromy around 0 , and therefore has eigenvalue -1 . It follows that

$$
\left(l^{2}-\frac{1}{4}\right)+\sum_{p \in \mathscr{P}} v_{p}\left(v_{p}+1\right)=L^{2}-\frac{1}{4} \text { for some } L \in \mathbb{Z}_{\geqq 0},
$$

obviously $L>l$ if $\mathscr{P} \neq \phi$. Let $\phi_{0}$ be the solution which is recessive at $x=\infty$ : $\phi_{0}(x) \sim x^{\frac{1}{2}-L}$ as $x \rightarrow \infty$. $\phi_{0}$ is an eigenvector for the monodromy around $\infty$, and therefore also for the monodromy around 0 . That is, it has no logarithm terms as $x \rightarrow 0$, and it is a multiple of a function of the form (3.11) for $p=0$. Because $\phi_{0}$ is meromorphic at the other poles $p \in \mathscr{P}$ of $V$, the conclusion is that $\partial_{x} \phi_{0}(x) / \phi_{0}(x)$ is meromorphic at all poles of $V$ and has finite order of growth for $x \rightarrow \infty$, so it is rational. The potential

$$
\tilde{V}(x)=V(x)-2 \partial_{x}\left[\partial_{x} \phi_{0}(x) / \phi_{0}(x)\right],
$$

obtained by applying the Darboux transformation using $\phi_{0}$, is again rational. Because $L^{2}-\frac{1}{4}+2\left(\frac{1}{2}-L\right)=(L-1)^{2}-\frac{1}{4}$, we get

$$
\tilde{V}(x) \sim \frac{\tilde{L}^{2}-\frac{1}{4}}{x^{2}} \text { for } x \rightarrow \infty \text { with } \tilde{L}=L-1,
$$

and because $l^{2}-\frac{1}{4}+2\left(\frac{1}{2} \pm l\right)=(l \pm 1)^{2}-\frac{1}{4}$ :

$$
\tilde{V}(x) \sim \frac{\tilde{l}^{2}-\frac{1}{4}}{x^{2}} \text { for } x \rightarrow 0 \text { with } \tilde{l}=l \pm 1 .
$$

Noting that $V$ is even, the function $\hat{\phi}_{0}(x)$ obtained from $\phi_{0}(x)$ by analytically continuing $\phi_{0}$ as $x$ walks around 0 to $-x$, and then reflecting back, is again a solution which is recessive at $\infty$. So $\hat{\phi}_{0}$ is a constant multiple (in fact, $\pm i$ times) of $\phi_{0}$ and the Darboux transformation with $\hat{\phi}_{0}$ instead of $\phi_{0}$ leads to the same result. It follows that $\tilde{V}(x)$ is an even function of $x$ again.

Finally, the eigenfunctions for $-\partial_{x}^{2}+\tilde{V}(x)$ (for the eigenvalue $k^{2}$ ) are given by $\left(\partial_{x}-\phi_{0}^{\prime} / \phi_{0}\right) \phi(x, k)$ if $\phi(x, k)$ are the eigenfunctions for $-\partial_{x}^{2}+V(x)$, so they are single-valued around all the poles $\tilde{p} \neq 0$ of $\tilde{V}$. Therefore, $\tilde{V}$ is again a potential satisfying (4.45), (4.46).

Now

$$
\begin{aligned}
\sum_{\tilde{p}} v_{\tilde{p}} \cdot\left(v_{\tilde{p}+1}\right)-\sum_{p} v_{p} \cdot\left(v_{p}+1\right) & =\left[\left(\tilde{L}^{2}-\frac{1}{4}\right)-\left(L^{2}-\frac{1}{4}\right)\right]-\left[\left(\tilde{l}^{2}-\frac{1}{4}\right)-\left(l^{2}-\frac{1}{4}\right)\right] \\
& =-2(L- \pm l)<0,
\end{aligned}
$$

so after finitely many applications of this procedure we arrive at a potential $\tilde{V}$ satisfying $\sum_{p} v_{\tilde{p}}\left(v_{\tilde{p}}+1\right) \leqq 0$, which, in view of $v_{\tilde{p}}\left(v_{\tilde{p}}+1\right)>0$ for all $\tilde{p}$, just means that $\tilde{P}=\phi$, that is $\tilde{V}(x)=\frac{\tilde{l}^{2}-\frac{1}{4}}{x^{2}}$ for some $\tilde{l} \in \mathbb{Z}_{\geqq 0}$. 
Because $1 / \phi_{0}$ is a solution of $\left(-\partial_{x}^{2}+\tilde{V}(x)\right) \phi=0$ and the Darboux transformation applied to $\widetilde{V}$ using $1 / \phi_{0}$ gives $V$ back, we have proved that $V$ is obtained from $\frac{\mathscr{L}^{2}-\frac{1}{4}}{x^{2}}$ for some $\mathscr{L} \in \mathbb{Z}_{\geqq 0}$ by finitely many rational Darboux transformations. Conversely, that any rational Darboux transformation leaves the set of potentials satisfying (4.45), (4.46) invariant is now quite clear in the light of the above arguments: for a Darboux transformation to be rational it is necessary to take an eigenfunction which at the same time is an eigenvector for the monodromy, that is, it does not have logarithmic terms.

We now prove that if we apply $\mu$ Darboux transformations, with $\mu<\mathscr{L}$, to $\tilde{V}(x)$ $=\frac{\mathscr{L}^{2}-\frac{1}{4}}{x^{2}}$, taking at each step any eigenfunction for the eigenvalue 0 which is dominant at $x=0$, then we arrive at a potential $V$ such that

i) $V(x) \sim \frac{l^{2}-\frac{1}{4}}{x^{2}}$ as $x \rightarrow 0, l=\mathscr{L}-\mu$, and

ii) The solutions of $\left(-\partial_{x}^{2}+V(x)\right) \phi=0$ have no logarithmic terms and have the asymptotic expansion

$$
\phi(x) \sim t \cdot x^{\frac{1}{2}-l}+u \cdot x^{\frac{1}{2}+l}+O\left(x^{\frac{1}{2}+l+1}\right) \text { as } x \rightarrow 0 .
$$

The proof is by induction on $\mu$. Note that if $\tilde{V}$ is the potential obtained from $V$ by means of the Darboux transformation using $\phi$, then the image of $\partial_{x}-\phi^{\prime} / \phi$ : $\operatorname{Ker}\left(-\partial_{x}^{2}+V\right) \rightarrow \operatorname{Ker}\left(-\partial_{x}^{2}+\tilde{V}\right)$ is one-dimensional because $\left(\partial_{x}-\phi^{\prime} / \phi\right) \phi=0$. So (only) for the eigenvalue 0 it is not true that all eigenfunctions of $-\partial_{x}^{2}+\widetilde{V}$ are obtained by applying $\partial_{x}-\phi^{\prime} / \phi$ to the eigenfunctions of $-\partial_{x}^{2}+V$. In this case one proceeds somewhat differently: one checks that $\tilde{\phi}=1 / \phi$ is a solution of $\left(-\partial_{x}^{2}+\tilde{V}\right) \phi=0$, and a linearly independent one $\tilde{\psi}$ is obtained by solving the Wronskian equation $\tilde{\phi} \cdot \partial_{x} \tilde{\psi}-\partial_{x} \tilde{\phi} \cdot \tilde{\psi}=1$. The result is

$$
\begin{aligned}
\tilde{\psi}(x)= & \frac{1}{\phi(x)} \cdot \int^{x} \phi(y)^{2} d y \\
= & \left(t x^{\frac{1}{2}-l}+u \cdot x^{\frac{1}{2}+l}+O\left(x^{\frac{3}{2}+l}\right)\right)^{-1} \\
& \cdot \int^{x}\left(t y^{\frac{1}{2}-l}+u \cdot y^{\frac{1}{2}+l}+O\left(y^{\frac{3}{2}+l}\right)\right)^{2} d y \\
= & \left(t x^{\frac{1}{2}-l}+u \cdot x^{\frac{1}{2}+l}+O\left(x^{\frac{3}{2}+l}\right)\right)^{-1} \\
& \cdot \int^{x}\left(t^{2} y^{1-2 l}+2 t u \cdot y+O\left(y^{3}\right)\right) d y \\
= & \left(t x^{\frac{1}{2}-l}+u \cdot x^{\frac{1}{2}+l}+O\left(x^{\frac{3}{2}+l}\right)\right)^{-1} \cdot\left(\frac{t^{2}}{2-2 l} x^{2-2 l}+v+O\left(x^{2}\right)\right) \\
= & \frac{t}{2-2 l} x^{\frac{3}{2}-l}+\frac{v}{t} x^{-\frac{1}{2}+l}+O\left(x^{\frac{1}{2}+l}\right) \text { as } x \rightarrow 0,
\end{aligned}
$$

which shows at the same time that no logarithmic terms appear and that (4.51) holds with other constants instead of $t, u$, and with $l$ replaced by $l-1$.

However, the formula also shows that approximating the recessive solution at $x=0$ of $\left(-\partial_{x}^{2}+\tilde{V}\right) \phi=0$ amounts to letting $t \rightarrow 0$, thereby choosing $v$ of order $t$. But this is the same as approximating the recessive solution at $x=0$ of $\left(\partial_{x}^{2}+V\right) \phi=0$. So any sequence of rational Darboux transformations starting at $\frac{\mathscr{L}^{2}-\frac{1}{4}}{x^{2}}$ can be 
replaced, with the same final result, by first performing a sequence of Darboux transformations using only recessive solutions at $x=0$, and then a sequence using only dominant solutions at $x=0$. Because the recessive solutions of $\left(-\partial_{x}^{2}+\frac{\mathscr{L}^{2}-\frac{1}{4}}{x^{2}}\right) \phi$ are equal to $\phi(x)=$ const $\cdot x^{\frac{1}{2}+\mathscr{L}}$, it leads again to a Bessel potential $V(x)=\frac{\mathscr{L}^{2}-\frac{1}{4}}{x^{2}}$ with $\mathscr{L}=\mathscr{L}+1$. This proves the first two statements of the proposition.

Remark. In terms of (4.47), the potentials $V$ described in the first two sentences of Proposition 4.3 satisfy $L=l+2 \mu$, where $\mu$ denotes the number of parameters in the family.

Starting at the Bessel potentials $(L=l)$, the solution of $\left(\partial_{x}^{2}+V\right) \phi=0$ which is recessive at $x=0$ is dominant at $x=\infty$. Performing the Darboux transformation with this solution leads to $(L, L) \rightarrow(L+1, L+1)$. The solution which is recessive at $\infty$, is dominant at $0:(L, L) \rightarrow(L-1, L-1)$. All other solutions (the generic ones) are dominant at both $x=\infty$ and at $x=0:(L, L) \rightarrow(L+1, L-1)$.

Because each of the recessive solutions (at $x=0$ or at $x=\infty$ ) can be approximated with dominant ones, it follows that both $(L+1, L+1)$ and $(L-1, L-1)$ can be approximated by the $(L+1, L-1)$ potentials. This will be denoted by the arrows $(L+1, L-1) \longrightarrow(L+1, L+1)$ and $(L+1, L-1) \longrightarrow(L-1, L-1)$.

At an $(L, l)$-potential which is obtained by applying a Darboux transformation with a solution $\phi_{0}$ of the previous equation, which is dominant at both $x=\infty$ and at $x=0$, we get that $\phi_{0}^{-1}$ is a solution of the equation at hand, which is recessive at both $x=\infty$ and at $x=0$. So from such $(L, l)$ we have only the Darboux transformations $(L, l) \rightarrow(L-1, l+1)$ if one uses the doubly recessive solution, or $(L, l) \rightarrow(L+1, l-1)$ in all other cases, because all other solutions are doubly dominant.

Because $(L, l) \longrightarrow(L, l+2)$, respectively $(L, l) \longrightarrow(L-2, l)$ transfers under $(L, l) \rightarrow(L+1, l-1) \quad$ to $\quad(L+1, l-1) \rightarrow(L+1, l+1), \quad$ respectively $(L+1, l-1) \longrightarrow(L-1, l-1)$, we get the following diagram:

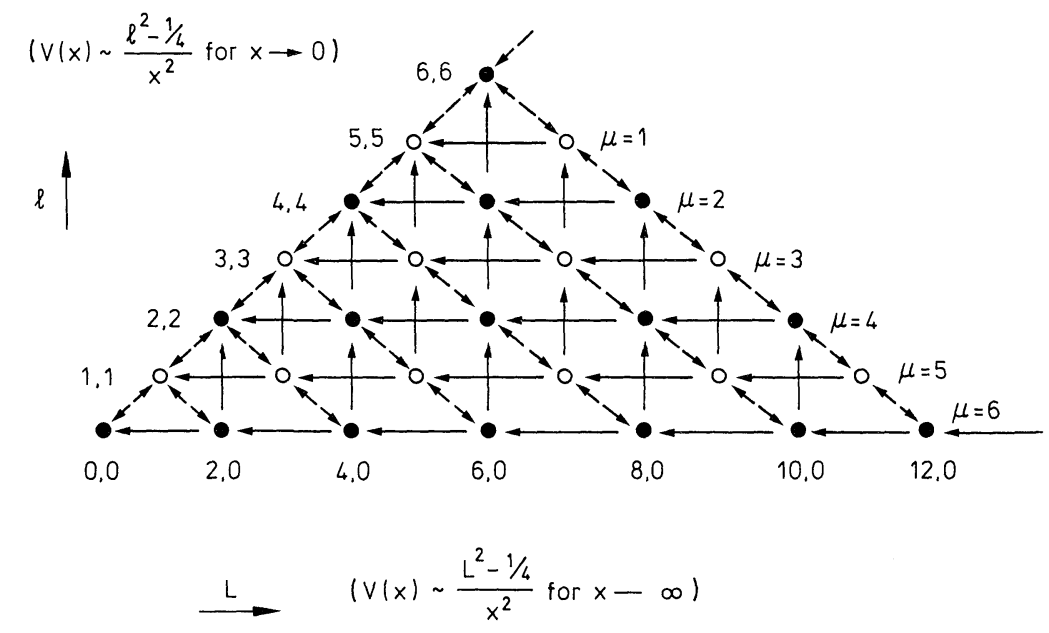

Diagram 4-1 
The limiting relations $\longrightarrow$ give two connected components: the $(L, l)$ with $L$ and $l$ both even, connected with $-\frac{1}{4} \cdot \frac{1}{x^{2}}$, and the $(L, l)$ with $L$ and $l$ both odd, connected with $\frac{3}{4} \cdot \frac{1}{x^{2}}$. These are denoted by closed, respectively open, dots in the diagram. A rational Darboux transformation interchanges the two components. Note that if we allow translations in the $x$-variables, then the limiting relations are enriched. If the pole of $V$, around which the eigenfunctions are not single-valued, runs to $\infty$, one arrives at limiting potentials $\hat{V}$ for which all eigenfunctions are meromorphic and the common eigenspace $\operatorname{Ker}\left(-\partial_{x}^{2}+V-\lambda\right) \cap \operatorname{Ker}(B-\Theta)$ is two-dimensional, so $\hat{V}(x)=v(v+1) /(x-a)^{2}$ for some $v \in \mathbb{Z}_{\geq 0}, a \in \mathbb{C}$. In order to actually get these, one can make a translation which shifts one of the $p \in \mathscr{P}$ to the origin and then let all the other poles run to $\infty$ by applying a homothety $x \mapsto \varepsilon \cdot x$. This leads to $\hat{V}(x)$ $=v_{p}\left(v_{p}+1\right) / x^{2}$. In the procedure of $(4.51), v_{p}$ becomes maximal if, after the first step, we take $\phi$ to be recessive at $p$ at each next step leading to $v_{p}=\mu$. So $v(v+1) / x^{2}$ can be obtained as a limit of translated potentials of Diagram 4.1 with $L=2 v$ or $L=2 v+1$.

For another refinement of Diagram 4.1, see Sect. 7.5.

\section{The Even Potentials Work Too}

We now turn to the question of whether the non-zero eigenfunctions $\phi(x, k)$ of $-\partial_{x}^{2}+V(x)$, with $V$ as in (4.45), (4.46), actually do satisfy a differential equation $\left(B\left(k, \partial_{k}\right)-\Theta(x)\right) \phi=0$ of positive order.

According to Proposition 4.3 we may assume that $V$ is obtained from $\frac{(l+\mu)^{2}-\frac{1}{4}}{x^{2}}$ by $\mu$ Darboux transformations, at each step using an eigenfunction for the eigenvalue 0 which is dominant at $x=0$. It will be convenient to view $\frac{(l+\mu)^{2}-\frac{1}{4}}{x^{2}}$ as obtained from $\frac{l^{2}-\frac{1}{4}}{x^{2}}$ by $\mu$ Darboux transformations, defined by eigenfunctions for the eigenvalue 0 which are recessive at $x=0$. Therefore, we can write

$$
\phi(x, k)=k^{-2 \mu}\left(\partial_{x}-v_{2 \mu}(x)\right) \circ \ldots \circ\left(\partial_{x}-v_{1}(x)\right) \cdot \psi(k x),
$$

where each $v_{i}(x)$ is rational in $x$, independent of $k[$ actually of the form $\left.\sum_{r} v_{j r}\left(x-x_{j r}\right)^{-1}\right]$ and $\psi$ is a non-zero solution of

$$
\left(-\partial_{y}^{2}+\frac{l^{2}-\frac{1}{4}}{y^{2}}-1\right) \cdot \psi(y)=0 .
$$

[See (1.35) for the appearance of $k x$ in $\psi$.]

This time, in contrast to the rational $\mathrm{KdV}$ case, $e^{-i k x} \phi(x, k)$ is not a rational function of $x$. Instead, at each step working away a second order derivative acting on $\psi$ in (5.1), using (5.2), we get that $\phi$ is a linear combination of $\psi(k x)$ and $\psi^{\prime}(k x)$ :

$$
\phi(x, k)=\alpha^{0}(x, k) \cdot \psi(k x)+\beta^{0}(x, k) \cdot \psi^{\prime}(k x),
$$

where the coefficients $\alpha^{0}(x, k), \beta^{0}(x, k)$ are rational functions of $x$ (and polynomials $\left.\operatorname{in} \frac{1}{k}\right)$. The coefficients in such a representation are uniquely determined because of 
Lemma 5.1. $\psi(y)$ and $\partial_{y} \psi(y)$ are linearly independent over the field of rational functions of $y$.

Proof. If $\partial_{y} \psi(y)=R(y) \psi(y)$ for a rational function $R$, then $\psi(y)=\exp \int^{y} R(z) d z$, combined with the asymptotics for $y \rightarrow \infty$ as treated in Sect. 3, gives us $\psi(y)$ $\sim c_{+} \cdot e^{i y}$ or $\psi(y) \sim c_{-} \cdot e^{-i y}$ as $y \rightarrow \infty$ in suitable sectors ( $c_{ \pm}$constants). It follows that $R(y) \sim \pm i+\sum_{k \geqq 2} c_{k} y^{-k}$ as $y \rightarrow \infty$, which in turn implies that $\psi$ is single-valued. But for (5.2) the monodromy has -1 as its only eigenvalue. This is a contradiction.

As we have seen in the proof of Proposition 4.3, $v_{j}(x)=\partial_{x} \phi_{j}(x) / \phi_{j}(x)$ is an odd function of $x$. Here $\left(-\partial_{x}^{2}+V_{j}(x)\right) \phi_{j}=0, V_{j}$ is an intermediate potential in the sequence of Darboux transformations. Since $\alpha^{0}(x, k)$, respectively $\beta^{0}(x, k)$, arise from the terms with an even, respectively odd, number of $\partial_{x}$ 's in (5.1), we get

$\alpha^{0}$ is an even function of $x$ and $k, \quad \alpha^{0}(x, k)=1+O\left(x^{-2}\right)$ as $x \rightarrow \infty$,

$\beta^{0}$ is an odd function of $x$ and $k, \quad \beta^{0}(x, k)=O\left(x^{-1}\right) \quad$ as $\quad x \rightarrow \infty$.

Our next step is to write

$$
\partial_{k}^{j} \phi(x, k)=\alpha^{j}(x, k) \cdot \psi(k x)+\beta^{j}(x, k) \cdot \psi^{\prime}(k x),
$$

where the $\alpha^{j}, \beta^{j}$ are recursively determined by

$$
\alpha^{j+1}=\partial_{k} \alpha^{j}-x \cdot \beta^{j}+\frac{l^{2}-\frac{1}{4}}{k^{2} x} \beta^{j}, \quad \beta^{j+1}=\partial_{k} \beta^{j}+x \cdot \alpha^{j}, \quad j \geqq 0,
$$

By induction on $j$ it follows that $\alpha^{j}(x, k), \beta^{j}(x, k)$ are rational in $x$ and polynomial in $k$. Furthermore, $x \mapsto \alpha^{j}(x, k)$ is even and $x \mapsto \beta^{j}(x, k)$ is odd. [Also: $\alpha^{j}(x,-k)$ $=(-1)^{j} \alpha^{j}(x, k), \beta^{j}(x,-k)=(-1)^{j+1} \beta^{j}(x, k)$.]

Lemma 5.2. For every even polynomial $\Theta$ of degree $m$ there are unique $B_{j}(k)$, $j=m, m-1, \ldots, 0$, such that

$$
\sum_{j=0}^{m} B_{j}(k) \cdot \alpha^{j}(x, k)-\Theta(x) \cdot \alpha^{0}(x, k) \rightarrow 0 \quad \text { as } \quad x \rightarrow \infty
$$

and

$$
\sum_{j=0}^{m} B_{j}(k) \cdot \beta^{j}(x, k)-\Theta(x) \cdot \beta^{0}(x, k) \rightarrow 0 \quad \text { as } \quad x \rightarrow \infty
$$

The $B_{j}(k)$ are polynomials in $\frac{1}{k}$ of degree $\leqq m-j$.

Proof. From (5.6), (5.4) we see by induction over $j$ that

$$
\begin{gathered}
\alpha^{2 r}(x, k) \sim(-1)^{r} \cdot x^{2 r}, \quad \alpha^{2 r-1}(x, k)=O\left(x^{2 r-2}\right) \quad \text { as } \quad x \rightarrow \infty, \\
\beta^{2 r-1}(x, k) \sim(-1)^{r-1} \cdot x^{2 r-1}, \quad \beta^{2 r-2}(x, k)=O\left(x^{2 r-3}\right) \quad \text { as } \quad x \rightarrow \infty .
\end{gathered}
$$

Assumed that the $B_{j}$ for $j>2 r$ have been determined such that the left-hand sides of (5.7), (5.8) are both $O\left(x^{2 r}\right)$ as $x \rightarrow \infty$. Then in view of (5.9), the condition that (5.7) is $O\left(x^{2 r-1}\right)$ is a linear inhomogeneous equation for $B_{2 r}$, involving the $B_{j}$ only for $j>2 r$, and with the coefficient in front of $B_{2 r}$ equal to $(-1)^{r}$. On the other hand, 
(5.8) is automatically $O\left(x^{2 r-1}\right)$ since it is an odd function of $x ; \beta^{j}$ and $\beta$ being odd and $\Theta$ being even.

Next assume that the $B_{j}$ for $j>2 r-1$ have been determined such that the lefthand sides of (5.7), (5.8) are both $O\left(x^{2 r-1}\right)$ as $x \rightarrow \infty$. Now (5.10) shows that the condition that (5.8) is $O\left(x^{2 r-2}\right)$ is a linear inhomogeneous equation for $B_{2 r-1}$ involving the $B_{j}$ only for $j>2 r-1$ and with coefficient in front of $B_{2 r-1}$ equal to $(-1)^{r-1}$. On the other hand, (5.7) is automatically $O\left(x^{2 r-2}\right)$, since it is an even function of $x: \alpha^{j}, \alpha$, and $\Theta$ all being even.

We now investigate the poles on the left-hand side of (5.7), (5.8).

Lemma 5.3. The $x \mapsto \alpha^{j}(x, k), x \mapsto \beta^{j}(x, k)$ for $j \geqq 0$ can only have poles at the poles $p \neq 0$ of $V$.

Proof. Writing $0=\left(-\partial_{x}^{2}+V(x)-k^{2}\right) \phi$ as a linear combination of $\psi$ and $\psi^{\prime}$ with rational coefficients, and using (5.3), (5.2), we get

$$
\begin{gathered}
-\partial_{x}^{2} \alpha^{0}+\left(V-\frac{l^{2}-\frac{1}{4}}{x^{2}}\right) \alpha^{0}+2 k \cdot \partial_{x} \beta^{0}+\frac{2}{k}\left(l^{2}-\frac{1}{4}\right)\left(\frac{\beta^{0}}{x^{3}}-\frac{\partial_{x} \beta^{0}}{x^{2}}\right)=0, \\
-\partial_{x}^{2} \beta^{0}+\left(V-\frac{l^{2}-\frac{1}{4}}{x^{2}}\right) \beta^{0}-2 k \cdot \partial_{x} \alpha^{0}=0 .
\end{gathered}
$$

From this second order system for $\left(\begin{array}{l}\alpha^{0} \\ \beta^{0}\end{array}\right)$ it is obvious that $\alpha^{0}, \beta^{0}$ can only have poles at the poles of $V$. We now have to show that they are regular at $x=0$ in spite of the singularity of the system there. Note, however, that $V-\frac{l^{2}-\frac{1}{4}}{x^{2}}$ is regular at $x=0$ (this is the reason for viewing $V$ as obtained from $\frac{l^{2}-\frac{1}{4}}{x^{2}}$ as in the beginning of this section), so the only problem is the term $\frac{\beta^{0}}{x^{3}}-\frac{\partial_{x} \beta^{0}}{x^{2}}$ in (5.11). Write

$$
\begin{gathered}
\alpha^{0}(x, k)=\sum_{j \geqq 0} \alpha_{j}^{0}(x) k^{-2 j}, \quad \alpha_{0}^{0}(x) \equiv 1, \\
\beta^{0}(x, k)=\sum_{j \geqq 0} \beta_{j}^{0}(x) k^{-2 j-1} .
\end{gathered}
$$

Then (5.11), respectively (5.12), read

$$
\begin{gathered}
-\partial_{x}^{2} \alpha_{j}^{0}+\left(V-\frac{l^{2}-\frac{1}{4}}{x^{2}}\right) \alpha_{j}^{0}+2 \partial_{x} \beta_{j+1}^{0}+2\left(l^{2}-\frac{1}{4}\right)\left(\frac{\beta_{j-1}^{0}}{x^{3}}-\frac{\partial_{x} \beta_{j-1}^{0}}{x^{2}}\right)=0, \\
-\partial_{x}^{2} \beta_{j}+\left(V-\frac{l^{2}-\frac{1}{4}}{x^{2}}\right) \beta_{j}^{0}-2 \partial_{x} \alpha_{j+1}^{0}=0 .
\end{gathered}
$$

Keeping in mind that $\alpha_{j+1}^{0}(\infty)=0, \beta_{j+1}^{0}(\infty)=0,(5.15)$ determines $\beta_{j+1}^{0}$ given $\alpha_{j}^{0}$ and $\beta_{j-1}^{0}$, whereas (5.16) determines $\alpha_{j+1}$ given $\beta_{j}$. So starting with $\alpha_{0}^{0}=1$, these equations can be used to determine all the $\alpha_{j}^{0}, \beta_{j}^{0}$ and thereby $\alpha^{0}, \beta^{0}$. If now $\beta_{j-1}^{0}$ is regular at $x=0$ then, because it is odd as a function of $x$, it can be written as $c \cdot x+O\left(x^{3}\right)$. Because $\frac{c \cdot x}{x^{3}}-\frac{\partial_{x}(c x)}{x^{2}}=0$, we read off from (5.15) that $\beta_{j+1}^{0}$ is regular at $x=0$ if $\alpha_{j}^{0}$ and $\beta_{j-1}^{0}$ are regular at $x=0$. Because it is obvious from (5.16) that 
$\alpha_{j+1}^{0}$ is regular at $x=0$ if $\beta_{j}^{0}$ is regular at $x=0$, by induction we get that $\alpha_{j}^{0}, \beta_{j}^{0}$ are regular at $x=0$ for all $j$, proving that $\alpha^{0}, \beta^{0}$ are regular at $x=0$.

That $\alpha^{j}, \beta^{j}$ can only have poles at the poles $p \neq 0$ of $V$ now follows from the inductive formulae in (5.6). Observe that $x^{-1} \beta^{j}$ is regular at $x=0$ if $\beta^{j}$ is regular at $x=0$, because $\beta^{j}$ is odd in $x$, so necessarily has a zero at $x=0$ then.

Theorem 5.4. Let $V$ be as in (4.45), (4.46). The non-zero eigenfunctions $\phi(x, k)$ in (5.1) satisfy an equation of the form $B\left(k, \partial_{k}\right) \phi(x, k)=\Theta(x) \phi(x, k)$, if and only if the polynomial $\Theta$ is even and satisfies

$$
\Theta^{(2 j-1)}(p)=0 \text { for all } 1 \leqq j \leqq v_{p}, \text { for each pole } p \in \mathbb{C} \backslash\{0\} \quad \text { of } V .
$$

Proof. The necessity is proved in the same way as the necessity of (3.34) in Theorem 3.5. Also the sufficiency can be proved along the same pattern as in the proof of Theorem 3.5, but for this more explanation is needed.

Let $\alpha(x, k)$, respectively $\beta(x, k)$, be the left-hand side of (5.7), respectively (5.8). From (5.11), (5.12) it follows that $\alpha^{0}, \beta^{0}$ have poles of order $\leqq v_{p}$ at the poles $p \neq 0$ of $V$, and from (5.6) we see that the same is true for $\alpha^{j}, \beta^{j}$ for all $j>0$. Now (5.7), (5.8) and Lemma 5.3 can be combined into the statement that

$$
\begin{aligned}
& \alpha(x, k)=\sum_{p \in \mathscr{P}} \sum_{r=0}^{v_{p}-1} \alpha_{p, r}(k) \cdot(x-p)^{r-v_{p}}, \\
& \beta(x, k)=\sum_{p \in \mathscr{P}} \sum_{r=0}^{v_{p}-1} \beta_{p, r}(k) \cdot(x-p)^{r-v_{p}} .
\end{aligned}
$$

From Proposition 3.3 we know that

$$
\partial_{x}^{2 j-1}\left[(x-p)^{v_{p}} \cdot \phi(x, k)\right]_{x=p}=0 \text { for } 1 \leqq j \leqq v_{p}, \quad p \in \mathscr{P} .
$$

This remains true if $\phi$ is replaced by $\left(B\left(k, \partial_{k}\right)-\Theta(x)\right) \phi$, assuming that $\Theta$ satisfies (5.17). Here we take $B\left(k, \partial_{k}\right)=\sum_{j=0}^{m} B_{j}(k) \partial_{k}^{j}$, with $B_{j}(k)$ as in Lemma 5.2. It follows that

$$
\begin{aligned}
& \partial_{x}^{2 j-1}\left[(x-p)^{v_{p}} \cdot\left\{\alpha(x, k) \cdot \psi(k x)+\beta(x, k) \cdot \psi^{\prime}(k x)\right\}\right]_{x=p}=0 \\
& \quad \text { for } 1 \leqq j \leqq v_{p}, \quad p \in \mathscr{P} .
\end{aligned}
$$

Expanding $\psi$, respectively $\psi^{\prime}$, at $x=p$, and inserting (5.18), (5.19), we get

$$
\begin{aligned}
& \sum_{r=0}^{2 j-1} \alpha_{p, r}(k) \cdot \frac{k^{2 j-1-r}}{(2 j-1-r) !} \cdot \psi^{(2 j-1-r)}(k p) \\
& \quad+\beta_{p, r}(k) \cdot \frac{k^{2 j-1-r}}{(2 j-1-r) !} \cdot \psi^{(2 j-r)}(k p)=0 \quad \text { if } \quad 2 j-1 \leqq v_{p}, \quad j \geqq 1,
\end{aligned}
$$

and

$$
\begin{aligned}
0= & \sum_{r=0}^{v_{p}} \alpha_{p, r}(k) \cdot \frac{k^{2 j-1-r}}{(2 j-1-r) !} \cdot \psi^{(2 j-1-r)}(k p) \\
& +\beta_{p, r}(k) \cdot \frac{k^{2 j-1-r}}{(2 j-1-r) !} \psi^{(2 j-r)}(k p)+O\left(k^{\left.2 j-1-v_{p}\right)}\right. \text { times } \\
& \alpha_{q, \tilde{r}}(k) \cdot \psi^{(s)}(k p) \text { or } \beta_{\tilde{q}, \tilde{r}}(k) \cdot \psi^{(\tilde{s})}(k p), \\
& \text { with } q, \tilde{q} \neq p, \text { if } 2 j-1 \geqq v_{p}, \quad j \leqq v_{p} .
\end{aligned}
$$


Now one gets by induction over $t$, from (5.2):

$$
\begin{gathered}
\psi^{(2 t)}(y)=\left((-1)^{t}+O\left(\frac{1}{y^{2}}\right)\right) \cdot \psi(y)+O\left(\frac{1}{y^{3}}\right) \psi^{\prime}(y), \\
\psi^{(2 t+1)}(y)=O\left(\frac{1}{y^{3}}\right) \cdot \psi(y)+\left((-1)^{t}+O\left(\frac{1}{y^{2}}\right)\right) \psi^{\prime}(y),
\end{gathered}
$$

where the order symbols represent polynomials in $\frac{1}{y}$ and the asymptotics is for $y \rightarrow \infty$. Inserting this in (5.22), (5.23), which we multiply by $k^{v_{p}-2 j} \cdot(-1)^{j}$, and where we substitute

$$
\left.\begin{array}{c}
\alpha_{p, 2 t} \cdot k^{v_{p}-1-2 t}(-1)^{-t-1}=\gamma_{p, 2 t} \\
\beta_{p, 2 t-1} \cdot k^{v_{p}-1-(2 t-1)}(-1)^{-t}=\gamma_{p, 2 t-1}
\end{array}\right\},
$$

and

$$
\left.\begin{array}{c}
\alpha_{p, 2 t-1} \cdot k^{v_{p}-1-(2 t-1)}(-1)^{-t}=\delta_{p, 2 t-1} \\
\beta_{p, 2 t} \cdot k^{v_{p}-1-2 t}(-1)^{-t}=\delta_{p, 2 t}
\end{array}\right\},
$$

to get

$$
\begin{aligned}
& \left(\sum_{r=0}^{v_{p}-1} \frac{1}{(2 j-1-r) !} \gamma_{p, r}(k)+O_{1}\left(\frac{1}{k}\right) \cdot\left(\begin{array}{l}
\gamma \\
\delta
\end{array}\right)\right) \psi^{\prime}(k p) \\
& +\left(\sum_{r=0}^{v_{p}-1} \frac{1}{(2 j-1-r) !} \delta_{p, r}(k)+O_{2}\left(\frac{1}{k}\right) \cdot\left(\begin{array}{l}
\gamma \\
\delta
\end{array}\right)\right) \cdot \psi(k p)=0
\end{aligned}
$$

for $1 \leqq j \leqq v_{p}, p \in \mathscr{P}$. Here we have used the convention that $\frac{1}{u !}=0$ if $u<0$, and by $O\left(\frac{1}{k}\right) \cdot\left(\begin{array}{l}\gamma \\ \delta\end{array}\right)$ we mean an expression which is linear in the $\gamma_{p, j}, \delta_{p, j}$, where $0 \leqq j \leqq v_{p}-1$ and $p$ runs through $\mathscr{P}$, with coefficients which are polynomials in $\frac{1}{k}$ without constant term.

The point now is that if we apply Lemma 5.1 to the left-hand side of (5.28) as a function of $k$, the fact that $\gamma_{p, r}, \delta_{p, r}$ are rational functions of $k$ implies that (5.28) gives twice the number of original equations:

$$
\begin{aligned}
& \sum_{r=0}^{v_{p}-1} \frac{1}{(2 j-1-r) !} \gamma_{p, r}(k)+O_{1}\left(\frac{1}{k}\right)\left(\begin{array}{l}
\gamma \\
\delta
\end{array}\right)=0, \\
& \sum_{r=0}^{v_{p}-1} \frac{1}{(2 j-1-r) !} \delta_{p, r}(k)+O_{2}\left(\frac{1}{k}\right)\left(\begin{array}{l}
\gamma \\
\delta
\end{array}\right)=0,
\end{aligned}
$$

for $1 \leqq j \leqq v_{p}, p \in \mathscr{P}$.

There are now as many equations as unknowns, $\gamma_{p, r}, \delta_{p, r}$, which can be written in the form

$$
(\mathscr{A}+\mathscr{B}(k)) \varepsilon=0, \quad \mathscr{B}(k)=O\left(\frac{1}{k}\right) \quad \text { as } \quad k \rightarrow \infty .
$$

Here $\varepsilon$ is the vector of all $\gamma_{p, r}, \delta_{p, r} \mathscr{A}=\left(\begin{array}{cc}A & 0 \\ 0 & A\end{array}\right)$ with $A$ as in (3.45). Because $A$ is invertible [cf. (3.44), (3.46)], $\mathscr{A}$ is invertible, $\mathscr{A}+\mathscr{B}(k)$ is invertible for large $|k|$, so 
$\varepsilon=0$ for large $|k|$. But this implies that $\left(B\left(k, \partial_{k}\right)-\Theta(x)\right) \phi(k, x)=0$ for large $|k|$, hence for all $k$ by analytic continuation.

The same observations that follow Theorem 3.5 apply here. For a given nonzero family $\phi(x, k)$ of eigenfunctions of $L=-\partial_{x}^{2}+V(x)$, the operators $B\left(k, \partial_{k}\right)$ such that $B\left(k, \partial_{k}\right) \phi=\Theta(x) \phi$ for some $\Theta$ form a commutative algebra $\mathscr{B}_{V}$, isomorphic to the algebra $\mathscr{B}_{V}$ of even polynomials $\Theta$ satisfying (5.17) by the map $B \mapsto \Theta . \mathscr{B}_{V}$ is also equal to the space of all $B=B\left(k, \partial_{k}\right)$ which commute with a given $B_{0} \in \mathscr{B}_{V}$ of positive degree.

Furthermore, every potential $V$ as in (4.45)-(4.46) with $v_{p}>1$ for some $p \in \mathscr{P}$ can be approximated by a potential $\tilde{V}$ of the same class but with $v_{p}=1$ for all poles $\tilde{p} \neq 0$ of $\tilde{V}$. The limit of $\mathscr{B}_{\tilde{V}}$ as $\tilde{V} \rightarrow V$ is equal to the set of even polynomials $\Theta$ such that $\Theta^{(j)}(p)=0$ for $1 \leqq j \leqq \frac{1}{2} v_{p}\left(v_{p}+1\right)$ for all poles $p \neq 0$ of $V$ and is strictly smaller than $\mathscr{B}_{V}$. In particular, $\mathscr{B}_{V}$ has elements of smaller positive degree than the $\mathscr{B}_{\tilde{V}}$ or $\lim _{\tilde{V} \rightarrow V} \mathscr{B}_{\tilde{V}}$. Introducing the analogue of the Adler-Moser polynomial [see (7.2.2)]:

$$
\theta(x)=\prod_{p \in \mathscr{P}}(x-p)^{\frac{1}{2} v_{p}\left(v_{p}+1\right)}
$$

or, using that $\theta$ is even, the simpler one

$$
\eta(y)=\prod_{\substack{\{p,-p\} \\ p \in \mathscr{P}}}\left(y-p^{2}\right)^{\frac{1}{2} v_{p}\left(v_{p}+1\right)}, \quad \theta(x)=\eta\left(x^{2}\right),
$$

the potentials $V$ for which $v_{p}>1$ for some $p \in \mathscr{P}$ form a hypersurface, determined by reading the discriminant equation for the polynomial $\eta$.

If $v_{p}=1$ for all $p \in \mathscr{P}$, then, writing $L=l+2 \mu$, there are $2 \mu(l+\mu)$ poles. This makes the minimal degree of a non-constant $\Theta$ equal to $2 \mu(l+\mu)+2$ $=\frac{1}{2} L^{2}-\frac{1}{2} l^{2}+2$. If $v_{p}>1$ for some $p \in \mathscr{P}$, then this minimal degree is smaller; see Sects. 7.2 and 7.5 for examples.

Finally, we make some remarks on the Bessel potentials $V(x)=\frac{c}{x^{2}}$. Because $x^{2} \in \mathscr{B}_{V}, \mathscr{B}_{V}$ contains all the even polynomials. The only cases when $\mathscr{B}_{V}$ is larger, that is, for which there exist $B^{ \pm}\left(k, \partial_{k}\right), \Theta$ such that $B^{ \pm}\left(k, \partial_{k}\right) \phi_{\infty}^{ \pm}=\Theta(x) \phi_{\infty}^{ \pm}$and $B^{-}$is not conjugate to $B^{+}$by a function of $k$, occur when $c=v(v+1)$ for some $v \in \mathbb{Z}_{\geqq 0}$. In this case $\mathscr{B}_{V}=\left\{\Theta ; \Theta^{(2 j-1)}(0)=0\right.$ for $\left.1 \leqq j \leqq v\right\}$. The larger $v$, the higher degree $\Theta \in \mathscr{B}_{V}$ must be in order that it not be an even polynomial. $\Theta(x)=x^{2 v+1}$ is the lowest possible case. For the operator $B$ see (7.4.14).

\section{6. $V(\infty)=\infty$ is the Airy Case}

Now assume that $(\operatorname{ad} L)^{m+1}(\Theta)=0$ for a non-constant $\Theta$, and that $V(\infty)=\infty$.

Lemma 6.1. $V(x) \sim \alpha \cdot x+\beta$ as $x \rightarrow \infty$ for some $\alpha, \beta \in \mathbb{C}, \alpha \neq 0$.

Proof. The coefficient of $\partial_{x}^{j}$ in $\left(\operatorname{ad}\left(\partial_{x}^{2}-V\right)\right)^{m+1}(\Theta)$ is a sum of terms of the form

$$
\Theta^{(l)} \cdot \prod_{i}\left(V^{\left(l_{i}\right)}\right)^{k_{i}}
$$

with non-negative integer coefficients, where

$$
\kappa=\sum_{i} k_{i}=\text { degree in } V \text { disregarding derivatives } \leqq(m+1-j) / 2
$$


and

$$
\begin{aligned}
\Lambda & =l+\sum_{i} k_{i} l_{i}=\text { total number of derivatives on } \Theta, V \\
& =2(m+1)-j-2 \kappa .
\end{aligned}
$$

The proof is obtained by observing that $\left(\operatorname{ad}\left(\partial_{x}^{2}-V\right)\right)^{m+1}=\left(\operatorname{ad} \partial_{x}^{2}-\operatorname{ad} V\right)^{m+1}$, and can be written as a sum of compositions

$$
\ldots \circ(-\operatorname{ad} V)^{m_{4}} \circ\left(\operatorname{ad} \partial_{x}^{2}\right)^{m_{3}} \circ(-\operatorname{ad} V)^{m_{2}} \circ\left(\operatorname{ad} \partial_{x}^{2}\right)^{m_{1}} .
$$

Then use the rules

$$
\begin{gathered}
{\left[\partial_{x}^{2}, a(x) \partial_{x}^{j}\right]=2 a^{\prime}(x) \partial_{x}^{j+1}+a^{\prime \prime}(x) \partial_{x}^{j}} \\
-\left[V, a(x) \partial_{x}^{j}\right]=a(x) \cdot \sum_{i=1}^{j}\left(\begin{array}{l}
j \\
i
\end{array}\right) \cdot V^{(i)} \partial_{x}^{j-i}
\end{gathered}
$$

Because ad $\partial_{x}^{2}$ increases the order by $\leqq 1$ and - ad $V$ lowers it, we need, in order to get a non-zero contribution, that $m_{2} \leqq m_{1}, m_{4} \leqq m_{1}-m_{2}+m_{3}, m_{6} \leqq m_{1}-m_{2}+m_{3}$ $-m_{4}+m_{5}$, etc. More precisely, (6.4) applied to $\Theta$ is a sum of terms of order (in $\partial_{x}$ ) $j \leqq \sum m_{\text {odd }}-\sum m_{\text {even }}$. Noting that

$$
\sum m_{\text {odd }}+\sum m_{\text {even }}=m+1, \quad \sum m_{\text {even }}=\kappa,
$$

(6.2) follows.

To see (6.3), note that (6.5) implies that if $\operatorname{ad} \partial_{x}^{2}$ is applied (increasing $m$ by 1 ) then $\kappa$ does not change, whereas $\Lambda$ is increased by 2 if $j$ stays the same, and increased by 1 if $j$ is increased by 1 . On the other hand, applying - ad $V$ (again increasing $m$ by 1 ) increases $\kappa$ by $1, \Lambda$ by $i$ if $j$ is decreased by $i$. In both cases (6.3) is preserved.

Next observe that $\Theta$ is a polynomial, so (6.1) can only be non-zero if $l \leqq \operatorname{deg} \Theta$. Furthermore, the rationality of $V$ allows us to write

$$
\begin{gathered}
V(x) \sim V_{\text {pol }}(x)+\sum_{r \geqq 1} c_{-r} x^{-r} \text { for } x \rightarrow \infty \text { with } V_{\text {pol }} \text { a polynomial, } \\
\text { which we assume to be of degree } \geqq 2 .
\end{gathered}
$$

Using that the derivative of a constant is zero (and not a function of order $x^{-1}$ ) we get that (6.1) has growth order (for $x \rightarrow \infty$ ) equal to

$$
\begin{aligned}
\tau= & \operatorname{deg} \Theta+\kappa \cdot \operatorname{deg} V_{\text {pol }}-\Lambda \\
& \text { iff } l \leqq \operatorname{deg} \Theta, \quad l_{i} \leqq \operatorname{deg} V_{\text {pol }} \text { for all } i,
\end{aligned}
$$

and lower order in all other cases. Moreover, the coefficient of $x^{\tau}$ in the expansion for $x \rightarrow \infty$ is equal to a positive integer times the highest order coefficient of $\Theta$ times the $\kappa^{\text {th }}$ power of the highest order coefficient of $V$. By making a substitution of variables $x=$ const $\cdot \tilde{x}, \lambda=$ const $\cdot \tilde{\lambda}$, one can arrange that the highest order coefficient of $V$ is equal to 1 . Using (6.3) we see that $\tau=\operatorname{deg} \Theta-2(m+1)+j$ $+\kappa \cdot\left(\operatorname{deg} V_{\text {pol }}+2\right)$, so if $\operatorname{deg} V_{\text {pol }}>2$, then $\tau$ is maximal if we choose $\kappa$ maximal.

If $m$ is even, we take $j=1, \kappa=\frac{m}{2}$ and we see that

$$
\operatorname{ad} \partial_{x}^{2} \circ(-\operatorname{ad} V) \circ \ldots \circ \operatorname{ad} \partial_{x}^{2} \circ(-\operatorname{ad} V) \circ \operatorname{ad} \partial_{x}^{2}(\Theta)
$$


gives a term $\Theta^{\prime} \cdot\left(V^{(2)}\right)^{\frac{m}{2}}$ in front of $\partial_{x}$ in $\left(\operatorname{ad}\left(\partial_{x}^{2}-V\right)\right)^{m+1}(\Theta)$ which has leading growth order as $x \rightarrow \infty$ and is not compensated by other terms, so we get a contradiction with $(\operatorname{ad} L)^{m+1}(\Theta)=0$.

If $m$ is odd, we take $j=0, \kappa=\frac{m+1}{2}$ and we see that

$$
(-\operatorname{ad} V) \circ \ldots \circ \operatorname{ad} \partial_{x}^{2} \circ(-\operatorname{ad} V) \circ \operatorname{ad} \partial_{x}^{2}(\Theta)
$$

gives a term $\Theta^{\prime} \cdot\left(V^{(2)}\right)^{\frac{m-1}{2}} V^{\prime}$, which is not compensated and again we get a contradiction. The lemma is proved.

By a substitution of variables $x=$ const $\cdot \tilde{x}+$ const, $\lambda=$ const $\cdot \tilde{\lambda}$, we can arrange that

$$
V(x) \sim x+\sum_{r \geqq 2} V_{r} \cdot x^{-r} \quad \text { as } \quad x \rightarrow \infty .
$$

(That $V_{1} x^{-1}$ does not occur follows from the rationality of $\int V$.) $x=\infty$ is an irregular singular point for $\left(-\partial_{x}^{2}+V(x)-\lambda\right) \phi=0$, this time with the leading term independent of $\lambda$. Define the sectors

$$
\begin{aligned}
& S^{+}=\left\{x \in \mathbb{C} ;|x| \text { large, } \varepsilon<\frac{3}{2} \arg x<\pi-\varepsilon\right\}, \\
& S^{-}=\left\{x \in \mathbb{C} ;|x| \text { large, }-\pi+\varepsilon<\frac{3}{2} \arg x<-\varepsilon\right\} .
\end{aligned}
$$

Then there are unique solutions $x \mapsto \phi^{ \pm}(x, \lambda)$ of $(L-\lambda) \phi=0$ with the asymptotic expansion

$$
\begin{gathered}
\phi^{ \pm}(x, \lambda) \sim e^{ \pm\left(\frac{2}{3} x^{3 / 2}-\lambda x^{1 / 2}\right)} \cdot x^{-1 / 4} \sum_{r \geqq 0} d_{r}^{ \pm}(\lambda) x^{-r / 2} \\
\text { as } x \rightarrow \infty \quad \text { in } S^{ \pm}, \quad d_{0}^{ \pm}=1,
\end{gathered}
$$

the expansion actually being valid in larger overlapping sectors in the usual way.

$A$ by now familiar application of Lemma 1.3 leads to the existence of unique $A_{s}^{ \pm}(\lambda), s=0, \ldots, m$ such that

$$
A^{ \pm}\left(\lambda, \partial_{\lambda}\right) \phi^{ \pm}(x, \lambda) \underset{\operatorname{def}}{\bar{~}} \sum_{s=0}^{m} A_{s}^{ \pm}(\lambda) \partial_{\lambda}^{s} \phi^{ \pm}(x, \lambda)=\Theta(x) \cdot \phi^{ \pm}(x, \lambda) .
$$

It follows immediately from (6.14), (6.15) that $m$ is even and $\operatorname{deg} \Theta=m / 2$.

Lemma 6.2. $A^{+}\left(\lambda, \partial_{\lambda}\right)=A^{-}\left(\lambda, \partial_{\lambda}\right)$.

Proof. Indeed, for one choice of the square root of $x$ in the asymptotic expansion (6.14) the coefficients are uniquely determined from the equation $\left(-\partial_{x}^{2}+V(x)-\lambda\right) \phi=0$. Then the single-valuedness of $V$ shows that substituting the other square root one again obtains a formal expansion which satisfies the same equation. This shows that

$$
d_{r}^{-}(\lambda)=(-1)^{r} d_{r}^{+}(\lambda)
$$

On the other hand, $(6.14),(6.15)$ lead to

$$
\sum_{\substack{j, s \\
j \leqq 0 \\
0 \leqq s \leqq m}} A_{q+s+j}^{ \pm}(\lambda) \cdot\left(\begin{array}{c}
q+s+j \\
s
\end{array}\right) \cdot(\mp 1)^{q+j} \cdot \partial_{\lambda}^{s} d_{j}^{ \pm}(\lambda)=\sum_{r=0}^{m / 2} \Theta_{r} \cdot d_{2 r-q}^{ \pm}(\lambda) .
$$


These equations for $q=m, m-1, \ldots, 0$ determine the $A_{j}^{ \pm}$for $j=m, m-1, \ldots, 0$ successively.

Now changing the sign in \pm amounts to multiplication by $(-1)^{q}$ on the righthand side and the same is true for each coefficient following the $A_{q+s+j}^{ \pm}$on the lefthand side. So it follows by downward induction that $A_{j}^{+}=A_{j}^{-}$for $j=m, m-1, \ldots, 0$.

From now on write $A\left(\lambda, \partial_{\lambda}\right)=A^{ \pm}\left(\lambda, \partial_{\lambda}\right)$. A somewhat closer look at the recurrent relations for the coefficients $d_{r}^{ \pm}(\lambda)$ in (6.14), which come from $\left(-\partial_{x}^{2}+V(x)-\lambda\right) \phi_{j}=0$, shows that the $d_{r}^{ \pm}(\lambda)$ are polynomials in $\lambda$ (of degree $\leqq 2 r$ ). It then follows from (6.17) that the coefficients of $A$ are polynomials in $\lambda$ as well. So this time the spaces $\mathscr{S}_{(x, y)}$ of germs at $(x, \lambda)$ of common solution $\phi$ of $(L-\lambda) \phi=0$, $(A-\Theta(x)) \phi=0$ define a holomorphic complex two-dimensional vector bundle $\mathscr{S}$ over $(\mathbb{C} \backslash\{$ poles of $V\}) \times \mathbb{C}$. The analytic continuation of solutions defines a flat holomorphic connection in $\mathscr{S}$. As in the even case, (4.18)-(4.33), we get for $\phi \in \operatorname{Ker}(L-\lambda) \cap \operatorname{Ker}(A-\Theta)$ :

$$
\partial_{\lambda}\left(\begin{array}{c}
\phi(x, \lambda) \\
\partial_{x} \phi(x, \lambda)
\end{array}\right)=Q(x, \lambda)\left(\begin{array}{c}
\phi(x, \lambda) \\
\partial_{x} \phi(x, \lambda)
\end{array}\right), \quad Q(x, \lambda)=\left(\begin{array}{ll}
a(x, \lambda) & b(x, \lambda) \\
c(x, \lambda) & d(x, \lambda)
\end{array}\right),
$$

where $a, b, c, d$ are rational functions of $x$, satisfying the compatibility equations.

$$
\partial_{\lambda} P+P Q=\partial_{x} Q+Q P, \quad P(x, \lambda)=\left(\begin{array}{cc}
0 & 1 \\
V(x)-\lambda & 0
\end{array}\right) .
$$

Writing these out, we get this time

$$
\begin{array}{ll}
\partial_{x} a=c-(V-\lambda) b, & \partial_{x} b=d-a, \\
\partial_{x} c=-1+(V-\lambda)(a-d), & \partial_{x} d=(V-\lambda) b-c .
\end{array}
$$

One can write $Q(x, \lambda)=\partial_{\lambda} \Phi(x, \lambda) \circ \Phi(x, \lambda)^{-1}$, where $\Phi(x, \lambda)$ is the fundamental solution

$$
\Phi(x, \lambda)=\left(\begin{array}{cc}
\phi_{j}^{+}(x, \lambda) & \phi_{j}^{-}(x, \lambda) \\
\partial_{x} \phi_{j}^{+}(x, \lambda) & \partial_{x} \phi_{j}^{-}(x, \lambda)
\end{array}\right)
$$

From (6.14) we see that $\operatorname{det} \Phi(x, \lambda) \rightarrow-2$ as $x \rightarrow \infty$, and because $x \mapsto \operatorname{det} \Phi(x, \lambda)$ is constant (trace $P=0$ ):

$$
\operatorname{det} \Phi(x, \lambda)=-2 .
$$

So $a+d=\operatorname{Tr} Q(x, \lambda)=\partial_{\lambda} \operatorname{det} \Phi(x, \lambda) / \operatorname{det} \Phi(x, \lambda)=0$. Inserting this in (6.20) then leads to the differential equation

$$
-\frac{1}{2} \partial_{x}^{3} b+2(V(x)-\lambda) \cdot \partial_{x} b+\partial_{x} V \cdot b+1=0 .
$$

From $Q(x, \lambda)=\partial_{\lambda} \Phi(x, \lambda) \circ \Phi(x, \lambda)^{-1}$ one also sees that

$$
b(x, \lambda)=-\frac{1}{2}\left(-\partial_{\lambda} \phi_{j}^{+} \cdot \phi_{j}^{-}+\partial_{\lambda} \phi_{j}^{-} \cdot \phi_{j}^{+}\right) \rightarrow-1 \quad \text { as } \quad x \rightarrow \infty,
$$

again using (6.14). So we can write

$$
b(x, \lambda) \sim-1+\sum_{s \geqq 1} b_{s}(\lambda) x^{-s} \text { as } x \rightarrow \infty,
$$


the series actually being convergent because of the rationality of $x \mapsto b(x, \lambda)$. Substituting (6.25), (6.12) in (6.23) and collecting the coefficients of $x^{-\sigma-1}$, we get

$$
\begin{aligned}
(2 \sigma-1) \cdot b_{\sigma}= & 2 \lambda \cdot b_{\sigma-1}(\sigma-1)+\frac{1}{2}(\sigma-3)(\sigma-2)(\sigma-1) b_{\sigma-3} \\
& -\sum_{r \geqq 2} V_{r} \cdot b_{\sigma-1-r} \cdot(2 \sigma-r-2)+(\sigma-1) V_{\sigma-1} \text { for all } \sigma \geqq 1 .
\end{aligned}
$$

Now suppose that $V_{r} \neq 0$ for some $r \geqq 2$, let $r_{0}$ be the smallest such $r$. It follows that $b_{\sigma}=0$ for $\sigma \leqq r_{0}$, and $b_{r_{0}+1+j}$ is a polynomial in $\lambda$ of degree $j$ with non-zero coefficient in front of $\lambda^{j}$.

Now from (6.23) we see that $b$ can only have poles at the poles of $V$, which do not depend on $\lambda$. So the rational function $x \mapsto b(x, \lambda)$ can be written as $b(x, \lambda)+1$ $=A(x, \lambda) / B(x)$, where $x \mapsto A(x, \lambda)$ is a polynomial and $B$ is a polynomial not dependent on $\lambda$. However, $A(x, \lambda)=B(x) \cdot \sum_{s \geqq 1} b_{s}(\lambda) x^{-s}$ shows that the (finitely many) coefficients of the polynomial $x \mapsto A(x, \lambda)$ are linearly dependent on only finitely many of the $b_{s}(\lambda)$, so the coefficients of $x \mapsto A(x, \lambda)$ are polynomials in $\lambda$, with a finite maximal degree. This is in contradiction with the appearance of arbitrary high powers of $\lambda$ in the coefficients of the expansion of $A(x, \lambda) / B(x)$ for $x \rightarrow \infty$. The conclusion is that $V_{r}=0$ for all $r \geqq 2$, and we have proved:

Theorem 6.3. If $(\operatorname{ad} L)^{m+1}(\Theta)=0$ for a non-constant $\Theta, V(\infty)=\infty$, then $V(x)$ $=\alpha x+\beta$ for some constants $\alpha, \beta$ (the Airy potential).

Because we have seen that in the Airy case there is a second order equation $A\left(\lambda, \partial_{\lambda}\right) \phi=\Theta(x) \cdot \phi$ with $\Theta(x)=x$, cf. (1.36), we get that in this case the algebra $\mathscr{B}_{V}$ of allowed $\Theta$ 's consists of all polynomials $\Theta$. The operator $A$ corresponding to $\Theta$ is then equal to $\Theta\left(\alpha \partial_{\lambda}^{2}+\frac{\lambda}{\alpha}-\beta \alpha\right)$.

\section{Some Illustrative Examples}

The purpose of this section is to display a number of examples of the theory presented so far, as well as to illuminate some points that have received only limited attention in the general treatment.

Most of these computations have been carried out with the assistance of Vaxima - a symbol manipulator at Berkeley. The help of the computer in this regard has been significant: both in providing an independent check on some of the results obtained by pure thought, as well as in producing a mass of "experimental data" which motivated and guided the development of the theory.

It is important to realize that, even for the computer, most computations are impossible. For example, with Vaxima we were able to solve for all the solutions of $\operatorname{ad} L^{m+1}(\Theta)=0$ for $m=4$. This provided a good start only because we could recognize the $\mathrm{KdV}$ equation lurking in the background. The same direct approach for $m=6$ would be impossible for the computer; however, we present here a complete analysis of $m=6$ which uses a very small part of the theory developed above plus the power of the computer (see Subsect. 7.3).

The examples given below are important because we do not have a general expression for $B\left(k, \partial_{k}\right)$ even when the $v_{p}=1$ for $p \in \mathscr{P}$. In this case the possible $\Theta$ were given explicitly. An infinite sequence of operators $B\left(k, \partial_{k}\right)$ is given in Sect. 7.4. 
See (7.4.7), (7.4.12), (7.4.13), and (7.4.14). When $v_{p}>1$ for some $p \in \mathscr{P}$ we do not have a very useful description of all possible $\Theta$ - except for (3.34) and (5.17) - and we certainly do not have an explicit expression for the corresponding $B\left(k, \partial_{k}\right)$.

\subsection{The KdV Case}

7.1.1. The $\theta$ Functions and the Potentials. In Adler and Moser [2] the potentials $V$ obtained from $V=0$ by $v$ rational Darboux transformations are given by taking $V_{v}=-2 \partial_{x}^{2}\left(\log \theta_{v}\right)$, and solving $\theta_{v}$ from a recursive system of first order differential equations; see Sect. 7.2 for more details. At each step one integration constant is added as a parameter in the $\theta_{v}$. Then they prove that the $V_{v}$ are the same (modulo a translation in $x$ ) as the flow-outs of $V_{v}^{(0)}(x)=\frac{v(v+1)}{x^{2}}$ by the $\mathrm{KdV}$ hierarchy, and that the time variables of these $\mathrm{KdV}$ flows are related to the integration constants in $\theta_{v}$ by some birational transformation.

An explicit formula expressing $\theta_{v}$ directly in terms of the time variables of the $\mathrm{KdV}$-flows has been found by Sato, see Date et al. [7], Segal and Wilson [23], or Kac [16, Chap. 14, exercises]. The recipe is to consider the character

$$
\chi(g)=\frac{\operatorname{det}\left(\left(\varepsilon_{j}\right)^{2 i-1}\right)_{1 \leqq i, j \leqq \nu}}{\operatorname{det}\left(\left(\varepsilon_{j}\right)^{i-1}\right)_{1 \leqq i, j \leqq \nu}}
$$

of the irreducible representation of $\operatorname{GL}(v, \mathbb{C})$ characterized by the partition $[1,2, \ldots, v]$ of $\frac{1}{2} v(v+1)$, as a function of the

$$
p_{i}=\operatorname{trace}\left(g^{i}\right)=\sum_{j=1}^{v}\left(\varepsilon_{j}\right)^{i} .
$$

Here $\varepsilon_{1}, \ldots, \varepsilon_{v}$ denote the eigenvalues of $g \in \mathrm{GL}(\nu, \mathbb{C})$. It turns out that $\chi$ depends only on the $p_{2 j-1}$ with $1 \leqq j \leqq v$. It is a weighted homogeneous polynomial of degree $\frac{1}{2} v(v+1)$ in these variables if we give $p_{2 j-1}$ the weight $2 j-1$. Putting $p_{1}=x$ one gets $\theta_{v}$ and $p_{2 j-1}$ is the time variable for the $(j-1)^{\text {st }} \mathrm{KdV}$-flow, $2 \leqq j \leqq v$. (One may consider translation in $x$ as the $0^{\text {th }} \mathrm{KdV}$-flow; this has to be added to get the full family of rational $\mathrm{KdV}$ potentials.)

The first few $\theta$ 's are given by (up to a multiplicative constant):

$$
\begin{gathered}
\theta_{0}=1, \quad \theta_{1}=x \quad\left(x \equiv p_{1}\right), \quad \theta_{2}=x^{3}-p_{3}, \\
\theta_{3}=x^{6}-5 p_{3} x^{3}+9 p_{5} x-5 p_{3}^{2}, \\
\theta_{4}=x^{10}-15 p_{3} x^{7}+63 p_{5} x^{5}-225 p_{7} x^{3}+315 p_{3} p_{5} x^{2}-175 p_{3}^{3} x+225 p_{3} p_{7}-189 p_{5}^{2}, \\
\theta_{5}=x^{15}-35 p_{3} x^{12}+252 p_{5} x^{10}+175 p_{3}^{2} x^{9}-2025 p_{7} x^{8}+945 p_{3} p_{5} x^{7} \\
+11025 p_{9} x^{6}-1225 p_{3}^{3} x^{6}-14175 p_{3} p_{7} x^{5}-11907 p_{5}^{2} x^{5} \\
+33075 p_{3}^{2} p_{5} x^{4}-55125 p_{3} p_{9} x^{3}+42525 p_{5} p_{7} x^{3}-12250 p_{3}^{4} x^{3} \\
+70875 p_{3}^{2} p_{7} x^{2}-59535 p_{3} p_{5}^{2} x^{2}+99225 p_{5} p_{9} x-91125 p_{7}^{2} x \\
-11025 p_{3}^{3} p_{5} x-55125 p_{3}^{2} p_{9}+85050 p_{3} p_{5} p_{7}-35721 p_{5}^{3}+6125 p_{3}^{5} .
\end{gathered}
$$

The difference between this function and the one that would be obtained from the Adler and Moser [2] method, as slightly modified in Ablowitz and Airault [1], is 
given by

$$
-1225 p_{3}^{3} \theta_{3}
$$

Applying the Adler-Moser recipe to obtain the potentials from the "thetafunctions," i.e., the expression

$$
V(x)=-2 \frac{\partial^{2}}{\partial x^{2}} \log \theta
$$

one obtains

$$
\begin{aligned}
& V_{0}(x)=0 \\
& V_{1}(x)=\frac{2}{x^{2}} \\
& V_{2}(x)=\frac{6 x^{4}+12 p_{3} x}{\left(x^{3}-p_{3}\right)^{2}}=\frac{6 x^{4}+12 p_{3} x}{\theta_{2}^{2}(x)} \\
& V_{3}(x)=\frac{12 x^{10}-324 p_{5} x^{5}+450 p_{3}^{2} x^{4}-300 p_{3}^{3} x+162 p_{5}^{2}}{\theta_{3}^{2}(x)} .
\end{aligned}
$$

We stop here since the numerator in $V_{4}(x)$ would already take seven lines.

7.1.2. The Operators $B^{+}\left(k, \partial_{k}\right)$ and the Functions $\Theta(x), \phi^{ \pm}(x, k)$. We will exhibit here the results of picking $\Theta(x)$ according to the recipe [cf. (3.34)]

$$
\Theta^{\prime}(x)=\text { const } \cdot \theta(x),
$$

and computing the operator $B^{+}\left(k, \partial_{k}\right)$ corresponding to $\phi^{+}(x, k)$ according to Lemma 2.1. This recipe gives the minimal positive degree for $B\left(k, \partial_{k}\right)$ if every zero of $\theta(x)$ is simple, i.e., $v_{p} \equiv 1$, and this happens for generic values of the parameters.

In all these examples we have

$$
\phi^{+}(x, k)=e^{i x k} \frac{\theta\left(x-\frac{1}{i k}, p_{3}-\frac{1}{(i k)^{3}}, \ldots, p_{2 d+1}-\frac{1}{(i k)^{2 d+1}}\right)}{\theta(x)},
$$

an exact expression which was useful in our computations, see Date et al. [7] and Segal and Wilson [23]. Compare also (3.28)'.

(a) From $\theta_{2}=x^{3}-p_{3}$ we get $\Theta_{2}(x)=x^{4}-4 p_{3} x$, and $B^{+}\left(k, \partial_{k}\right)=\left(-\partial_{k}^{2}+\frac{6}{k^{2}}\right)^{2}$ $+4 i p_{3} \partial_{k}$ [compare (1.40)].

(b) From $\theta_{3}$ we get

$$
\Theta_{3}(x)=x^{7}+\frac{63}{2} p_{5} x^{2}-35 p_{3}^{2} x-\frac{35}{4} p_{3} x^{4}
$$

and $B^{+}\left(k, \partial_{k}\right)$ is

$$
\begin{aligned}
& i\left(\partial_{k}-\frac{3}{k}\right)\left(\partial_{k}-\frac{2}{k}\right)\left(\partial_{k}-\frac{1}{k}\right) \partial_{k}\left(\partial_{k}+\frac{1}{k}\right)\left(\partial_{k}+\frac{2}{k}\right)\left(\partial_{k}+\frac{3}{k}\right) \\
& -\frac{63}{2} p_{5}\left(\partial_{k}-\frac{1}{k}\right)\left(\partial_{k}+\frac{1}{k}\right)-35 i p_{3}^{2} \partial_{k}-\frac{35}{4} p_{3}\left(\left[\left(\partial_{k}-\frac{2}{k}\right)\left(\partial_{k}+\frac{2}{k}\right)\right]^{2}-\frac{35}{k^{4}}\right)
\end{aligned}
$$


Notice that in these two cases we can associate to each term in $\Theta$ a monomial in $B^{+}\left(k, \partial_{k}\right)$. This gets more complicated in the next example.

(c) From $\theta_{4}$ we get

$$
\begin{aligned}
\Theta_{4}= & x^{11}-\frac{165}{8} p_{3} x^{8}+\frac{231}{2} p_{5} x^{6}-\frac{2475}{4} p_{7} x^{4}+1155 p_{3} p_{5} x^{3} \\
& -\frac{1925}{2} p_{3}^{3} x^{2}-\left(2079 p_{5}^{2}-2475 p_{3} p_{7}\right) x
\end{aligned}
$$

and the operator $B^{+}\left(k, \partial_{k}\right)$ is given by

$$
\begin{aligned}
& \left(\partial_{k}-\frac{5}{k}\right)\left(\partial_{k}-\frac{4}{k}\right)\left(\partial_{k}-\frac{3}{k}\right)\left(\partial_{k}-\frac{2}{k}\right)\left(\partial_{k}-\frac{1}{k}\right) \partial_{k}\left(\partial_{k}+\frac{1}{k}\right) \\
& \cdot\left(\partial_{k}+\frac{2}{k}\right)\left(\partial_{k}+\frac{3}{k}\right)\left(\partial_{k}+\frac{4}{k}\right)\left(\partial_{k}+\frac{5}{k}\right) \\
& \quad-\frac{165}{8} p_{3}\left(\partial_{k}^{8}-\frac{56}{k^{2}} \partial_{k}^{6}+\frac{336}{k^{3}} \partial_{k}^{5}-\frac{840}{k^{4}} \partial_{k}^{4}+\frac{7560}{k^{6}} \partial_{k}^{2}-\frac{25200}{k^{7}} \partial_{k}+\frac{3528}{k^{8}}\right) \\
& \quad-\frac{231}{2} p_{5}\left(\partial_{k}^{6}-\frac{30}{k^{2}} \partial_{k}^{4}+\frac{120}{k^{3}} \partial_{k}^{3}-\frac{270}{k^{4}} \partial_{k}^{2}+\frac{360}{k^{5}} \partial_{k}\right) \\
& \quad-\frac{2475}{4} p_{7}\left(\partial_{k}^{4}-\frac{12}{k^{2}} \partial_{k}^{2}+\frac{24}{k^{3}} \partial_{k}\right)+1155 p_{3} p_{5} i\left(\partial_{k}^{3}-\frac{6}{k^{2}} \partial_{k}+\frac{6}{k^{3}}\right) \\
& +17325 i p_{3}^{2}\left(-\frac{1}{k^{4}} \partial_{k}+\frac{2}{k^{5}}\right)-\frac{1925}{2} p_{3}^{3}\left(-\partial_{k}^{2}+\frac{2}{k^{2}}\right)+\left(2079 p_{5}^{2}-2475 p_{3} p_{7}\right) i \partial_{k} .
\end{aligned}
$$

In contrast with the examples given earlier, the term in $B^{+}$with coefficient $p_{3}^{2}$ cannot be paired to any term in $\Theta_{4}$.

\subsection{The Even Case}

We present the first few examples in this family.

7.2.1. The $\theta$ Functions and the Potentials. The Darboux transformation from one potential to a new family of potentials requires the introduction, at stage $k$, of an eigenfunction $\phi_{k}$ with eigenvalue equal to zero.

Adler and Moser noticed that setting

$$
\phi_{k}=\frac{\theta_{k+1}}{\theta_{k}},
$$

the desired recursion relation among the successive $\phi_{k}$ can then be written as

$$
\theta_{k+1}^{\prime} \theta_{k-1}-\theta_{k+1} \theta_{k-1}^{\prime}=(2 k+1) \theta_{k}^{2} \text {. }
$$

They further noticed that the rational solutions of $\mathrm{KdV}$ are obtained if one starts with $\theta_{0}=1, \theta_{1}(x)=x$, and then puts $V(x)=-2\left(\log \theta_{k}\right)^{\prime \prime}$. We have found that the same idea can be applied in the even case. If one puts $\theta_{0}=1, \theta_{1}(x)=x^{1 / 2}$, and makes one more change [see (7.2.2) below], we get $\theta_{0}=1, \theta_{1}=x^{1 / 2}, \theta_{2}=x^{2}+t_{1}$. Another 
application of the recursion relation (7.2.1) with $t_{1} \neq 0$ would produce a logarithmic term which in turn would produce [see (7.2.2)], a nonrational potential $V(x)$. Therefore, we restrict $t_{1}$ to be zero and pick a new integration constant $t_{2}$ :

$$
\begin{aligned}
& \theta_{3}=\frac{3}{4} x^{9 / 2}+t_{2} x^{1 / 2}, \\
& \theta_{4}=\frac{15}{32} x^{8}+\frac{15 t_{2}}{4} x^{4}+t_{3} x^{2}-\frac{5}{2} t_{2}^{2} .
\end{aligned}
$$

Now setting both $t_{1}=t_{2}=0$, we get

$$
\theta_{5}=\frac{525 x^{25 / 2}}{2048}+\frac{35 t_{3} x^{13 / 2}}{8}+\frac{3 t_{4} x^{9 / 2}}{4}-\frac{7 t_{3}^{2} x^{1 / 2}}{3}
$$

and finally

$$
\begin{aligned}
\theta_{6}= & \frac{33075 x^{18}}{262144}+\frac{19845 t_{3} x^{12}}{2048}+\frac{945 t_{4} x^{10}}{256}+\frac{15 t_{5} x^{8}}{32}-\frac{2205 t_{3}^{2} x^{6}}{32} \\
& -\frac{63 t_{3} t_{4} x^{4}}{4}+t_{3} t_{5} x^{2}-\frac{9 t_{4}^{2} x^{2}}{5}-\frac{49 t_{3}^{3}}{2} .
\end{aligned}
$$

Setting some $t_{i}=0$ amounts to taking a step of the Darboux transformation with a recessive solution, while allowing $t_{i} \neq 0$ makes use of a dominant one.

Thus the method here is exactly the same as that described in Sect. 4.

Once the "theta functions" are obtained, one can adapt the Adler-Moser recipe to produce the corresponding potentials, with only one modification: the addition of the term $-\frac{1}{4 x^{2}}$. More explicitly, the potentials will be computed by

$$
V(x)=-\frac{1}{4 x^{2}}-2 \frac{\partial^{2}}{\partial x^{2}} \log \theta .
$$

The first few examples follow:

$$
\begin{gathered}
V_{0}(x)=-\frac{1}{4 x^{2}}, \quad V_{1}(x)=\frac{3}{4 x^{2}}, \quad V_{2}(x)=-\frac{1}{4 x^{2}}+\frac{4\left(x^{2}-t_{1}\right)}{\left(x^{2}+t_{1}\right)^{2}}, \\
V_{3}(x)=-\frac{1}{4 x^{2}}+\frac{1}{x^{2}}+\frac{72 x^{2}\left(x^{4}-4 t_{2}\right)}{\left(3 x^{4}+4 t_{2}\right)^{2}}, \\
V_{4}(x)=-\frac{1}{4 x^{2}}+16\left(225 x^{14}-900 t_{2} x^{10}-1560 t_{3} x^{8}+15600 t_{2}^{2} x^{6}+960 t_{2} t_{3} x^{4}\right. \\
\left.+256 t_{3}^{2} x^{2}+14400 t_{2}^{3} x^{2}+640 t_{2}^{2} t_{3}\right) /\left(15 x^{8}+120 t_{2} x^{4}+32 t_{3} x^{2}-80 t_{2}^{2}\right)^{2}, \\
V_{5}(x)=-\frac{1}{4 x^{2}}+\frac{1}{x^{2}}+72 x^{2}\left(826875 x^{20}-21168000 t_{3} x^{14}-9676800 t_{4} x^{12}\right. \\
+203212800 t_{3}^{2} x^{8}+20643840 t_{3} t_{4} x^{6}+2359296 t_{4}^{2} x^{4}+321126400 t_{3}^{3} x^{2} \\
\left.+22020096 t_{3}^{2} t_{4}\right) /\left(1575 x^{12}+26880 t_{3} x^{6}+4608 t_{4} x^{4}-14336 t_{3}^{2}\right)^{2} .
\end{gathered}
$$


7.2.2. The Operators $B\left(k, \partial_{k}\right)$ and the Functions $\Theta(x)$. Here we use the $\theta$ 's described above to exhibit one of the differential operators $B\left(k, \partial_{k}\right)$ predicted by the general theory. The recipe for $\Theta$ is, except for a multiplicative constant [cf. (5.17)],

$$
\Theta^{\prime}(x)=\Pi\left(x^{2}-x_{i}^{2}\right) x \quad \text { with } x_{i} \text { zeros of } \theta, \quad x_{i} \neq 0 .
$$

This gives, as in Sect. 7.1.2, the minimal positive degree for $B\left(k, \partial_{k}\right)$ if every zero of $\theta(x)$ is simple.

Since this time around we do not have a nice exact expression for the eigenfunctions $\phi^{ \pm}(x, k)$ but, on the other hand, we do have a two-dimensional common eigenspace, we have used for $\phi(x, k)$ the solution of $\left(-\partial_{x}^{2}+V-k^{2}\right) \phi=0$ that is recessive at $x=0$. The computation of $B$ was then carried out with Vaxima using the readily available Taylor expansion of $x^{-1 / 2} \phi(x, k)($ at $x=0)$ up to a high enough order. More precisely, $\phi$ can be expressed in terms of Bessel functions and their derivatives, and then these formulas are used to obtain the Taylor expansions.

(a) From $\theta_{2}=x^{2}+t_{1}$, we get $\Theta_{2}=x^{4}+2 t_{1} x^{2}$, and

$$
B_{2}\left(k, \partial_{k}\right)=\left(-\partial_{k}^{2}+\frac{15}{4 k^{2}}\right)^{2}+2 t_{1}\left(-\partial_{k}^{2}-\frac{1}{4 k^{2}}\right) \text {. }
$$

(b) From $\theta_{3}$, we get $\Theta_{3}=x^{6}+4 t_{2} x^{2}$, and

$$
B_{3}\left(k, \partial_{k}\right)=\left(-\partial_{k}^{2}+\frac{35}{4 k^{2}}\right)^{3}+4 t_{2}\left(-\partial_{k}^{2}+\frac{3}{4 k^{2}}\right) \text {. }
$$

(c) From $\theta_{4}$, we get

$$
\Theta_{4}=x^{10}+\frac{40 t_{2} x^{6}}{3}+\frac{16 t_{3} x^{4}}{3}-\frac{80 t_{2}^{2} x^{2}}{3},
$$

and for $B\left(k, \partial_{k}\right)$

$$
\begin{aligned}
& \left(-\partial_{k}^{2}+\frac{63}{4 k^{2}}\right)^{5}-\frac{80}{3} t_{2}^{2}\left(-\partial_{k}^{2}-\frac{1}{4 k^{2}}\right)+\frac{16}{3} t_{3}\left(-\partial_{k}^{2}+\frac{15}{4 k^{2}}\right)^{2} \\
& +\frac{40}{3} t_{2}\left[-\partial_{k}^{6}+\frac{93}{4 k^{2}} \partial_{k}^{4}-\frac{93}{k^{3}} \partial_{k}^{3}+\frac{3861}{16 k^{4}} \partial_{k}^{2}-\frac{1629}{4 k^{5}} \partial_{k}+\frac{19575}{k^{6}}\right]
\end{aligned}
$$

(d) From $\theta_{5}$, we get

$$
\Theta_{5}=x^{14}-\frac{14336}{225} t_{3}^{2} x^{2}+\frac{512}{75} t_{4} x^{6}+\frac{448}{15} t_{3} x^{8},
$$

and for $B\left(k, \partial_{k}\right)$

$$
\begin{gathered}
\left(-\partial_{k}^{2}+\frac{99}{4 k^{2}}\right)^{7}-\frac{14336}{225} t_{3}^{2}\left(-\partial_{k}^{2}+\frac{3}{4 k^{2}}\right)-\frac{512}{75} t_{4}\left(-\partial_{k}^{2}+\frac{35}{4 k^{2}}\right)^{3}+\frac{448}{15} t_{3} \\
\cdot\left[\partial_{k}^{8}-\frac{51}{k^{2}} \partial_{k}^{6}+\frac{306}{k^{3}} \partial_{k}^{5}-\frac{7965}{8 k^{4}} \partial_{k}^{4}+\frac{1845}{k^{5}} \partial_{k}^{3}-\frac{1395}{16 k^{6}} \partial_{k}^{2}-\frac{70335}{8 k^{7}} \partial_{k}+\frac{3856545}{256 k^{8}}\right]
\end{gathered}
$$




\subsection{The Cases $m \leqq 6(V(\infty)=0)$}

Here we give an account of a computer assisted approach to determine those $V(x)$ such that $B\left(k, \partial_{k}\right) \phi(x, k)=\Theta(x) \phi(x, k)$ with $m \equiv$ order $B \leqq 6$.

The cases $m=1,2,3$ have been handled in Sect. 1 , so we only look at $m=4,5,6$ here.

We use the notation of $(2.4),(2.5),(2.20),(2.21),(2.22)$, so that

$$
V(x)=\sum_{l \geqq 2} V_{l} x^{-l}, \quad \Theta(x)=\sum_{r=0}^{m} \theta_{r} x^{r},
$$

and

$$
B\left(k, \partial_{k}\right)=\sum_{p=-\infty}^{m} B_{p}(k) \partial_{k}^{p} .
$$

We consider solving (2.22) for all values of $q \leqq m$, including negative values, and the sum for $B$ is only formal. Using the relation

$$
V=\left(\frac{P}{\Theta^{\prime}}\right)^{\prime}, \quad \operatorname{deg} p \leqq 4, \quad \operatorname{deg} \Theta \leqq 6,
$$

one rules out $V_{2}=0$ and concludes that once $\theta_{r}, 1 \leqq r \leqq 6$, and $V_{l}, 2 \leqq l \leqq 6$, are found, then $P(x)$ and thus $V(x)$ are determined.

The bulk of the work consists of exploiting the equations resulting from $B_{p}(k)=0, p=-1,-2, \ldots,-5$, needed to make $B$ into a differential operator.

For an illustration, from $B_{-1}(k)=0$ we get

$$
\begin{gathered}
\theta_{1} V_{2}+\theta_{2} V_{3}+\ldots+\theta_{6} V_{7}=0 \\
6 \theta_{6}\left(V_{2} V_{5}-5 V_{5}+V_{3} V_{4}\right)+\theta_{5}\left(6 V_{2} V_{4}-20 V_{4}+3 V_{3}\right) \\
+3\left(V_{2}-2\right)\left(2 \theta_{4} V_{3}-\theta_{3} V_{2}\right)=0 \\
\left(V_{2}-6\right)\left(V_{2}-2\right)\left(3 V_{3} \theta_{6}+V_{2} \theta_{5}\right)=0
\end{gathered}
$$

The equations from $B_{-2}(k)=0$ are already too messy to give here. They can be used to prove the following:

Lemma 7.3.1. The linear homogeneous equations in $\theta_{1}, \ldots, \theta_{6}$ arising from $B_{-1}(k)$ $=B_{-2}(k)=0$ have a non-trivial solution if and only if

$$
\left(4 V_{2} V_{4}-3 V_{3}^{2}\right)\left(V_{2}-2\right)\left(V_{2}-6\right)\left(V_{2}-\frac{15}{4}\right)\left(V_{2}-\frac{35}{4}\right)=0 \text {. }
$$

This shows that there are five cases to consider. In each case we use $B_{-p}(k)$, $p=3,4,5$. The summary below ignores the instances $V(x)=a /(x-b)^{2}$, i.e. a Bessel potential.

I. Only the first factor in (7.3.4) vanishes: this leads to the potential $V_{3}(x)$ in Sect. 7.1.1 with $p_{3}=s^{3}, p_{5}=s^{5}$.

II. $V_{2}=2$ : gives nothing (i.e. only a Bessel potential).

III. $V_{2}=6$ : leads to $V_{2}(x)$ in Sect. 7.1.1.

IV. $V_{2}=\frac{15}{4}$ : leads to $V_{2}(x)$ in Sect. 7.2.1.

V. $V_{2}=\frac{35}{4}$ : leads to $V_{3}(x)$ in Sect. 7.2.1. 


\subsection{One Step from Bessel}

In this section we will exhibit, for each $m \in \mathbb{Z}_{>0}, m \geqq 6$, a potential $V$ and a corresponding differential equation $B\left(k, \partial_{k}\right) \phi=\Theta(x) \phi$ of minimal positive order equal to $m$. Let $V_{0}(x)=\frac{(v-1) v}{x^{2}}$ be the Bessel potential. The eigenfunctions for the eigenvalue 0 of $-\partial_{x}^{2}+V_{0}$ are, up to a constant factor, given by

$$
\phi_{0}(x)=t \cdot x^{1-v}+x^{v}, \text { respectively } \phi_{0}(x)=x^{1-\nu} .
$$

(For $v=\frac{1}{2}$ this has to be replaced by $t \cdot x^{1 / 2} \log x+x^{1 / 2}$, respectively $x^{1 / 2} \log x$.) Because

$$
\log \phi_{0}(x)=(1-v) \cdot \log x+\log \left(t+x^{2 v-1}\right),
$$

we see that $\phi_{0}^{\prime}(x) / \phi_{0}(x)$ is rational if and only if $2 v \in \mathbb{Z}$ (and $t=0$ if $v=\frac{1}{2}$ ), which we assume from now on. Of the two $v$ 's giving rise to the same $(v-1) v$, we will choose the positive one.

Applying the Darboux transformation we get the new potential

$$
V(x)=V_{0}(x)-2\left(\log \phi_{0}(x)\right)^{\prime \prime}=\frac{(v-2)(v-1)}{x^{2}}-2\left(\log \left(t+x^{2 v-1}\right)\right)^{\prime \prime}
$$

In the examples in 7.1 and 7.2 we recognize these as the families where all the parameters, except the one with the highest index, are equal to zero. Assume from now on that $v>\frac{1}{2}, t \neq 0$. Then $V(x)$ has $2 v-1$ non-zero poles, at the $(2 v-1)^{\text {th }}$ roots of $-t$, and each of them has $v_{p}=1$, i.e. the coefficient is equal to 2 .

Now $\Theta^{\prime}$, according to Theorem 3.5 , respectively 5.4 , must have zeros at the poles, that is,

$$
\Theta^{\prime}(x)=\left(t+x^{2 v-1}\right) \cdot R(x)
$$

for some polynomial $R(x)$. Furthermore, if $v \in \mathbb{Z}$, the derivatives at $x=0$ of $\Theta^{\prime}$ of order $2 j-2,1 \leqq j \leqq v-2$, all have to vanish, whereas for $v \notin \mathbb{Z}$ we have to add the condition that $\Theta$ is even. In both cases the $R$ of minimal degree with these properties is $R(x)=$ const $\cdot x$, that is, the $\Theta$ of minimal degree and with leading coefficient equal to 1 , is equal to

$$
\Theta_{t}(x)=x^{2 v+1}+\left(v+\frac{1}{2}\right) \cdot t \cdot x^{2} .
$$

Note that in the $\mathrm{KdV}$ case the order $2 v+1$ is lower than one would expect from just counting the number of equations in (3.34). There are $2 v-1+v-2=3 v-3$ equations for $\Theta^{\prime}$, so one would expect $3 v-2$ as the minimal order.

The eigenfunction $\phi_{\infty}^{+}(x, k)$ in (2.4) is given by

$$
\phi_{\infty}^{+}(x, k)=\frac{1}{i k}\left(\partial_{x}-\frac{1-v}{x}-\frac{(2 v-1) x^{2 v-2}}{t+x^{2 v-1}}\right) \cdot \psi(k x),
$$

where $\psi(y)$ is the eigenfunction of $-\partial_{y}^{2}+\frac{(v-1) v}{y^{2}}$ for the eigenvalue 1 such that $\psi(y) \sim e^{i y}$ as $y \rightarrow \infty, y=k x, x \in S_{\infty}^{+}(k)$ [cf. the remark preceding (1.35).]

From the proof of Lemma 2.1 it is obvious that the unique differential operator $B_{t}\left(k, \partial_{k}\right)$ such that $B_{t} \phi_{\infty}^{+}=\Theta_{t} \phi_{\infty}^{+}$depends smoothly on $t$, so that we can expand

$$
B_{t}\left(k, \partial_{k}\right)=\sum_{j=0}^{\infty} B^{(j)}\left(k, \partial_{k}\right) \cdot t^{j}
$$


Multiplying the equation $B \phi=\Theta \phi$ by $t+x^{2 v-1}$ in order to get rid of $t$ in the denominator, and collecting equal powers of $t$, we get [using that $(1-v)+(2 v-1)=v]$ :

$$
\begin{gathered}
B^{(j-1)} \frac{1}{i k}\left(\partial_{x}-\frac{1-v}{x}\right) \psi(k x)+B^{(j)} \frac{x^{2 v-1}}{i k}\left(\partial_{x}-\frac{v}{x}\right) \psi(k x)=0 \text { for } j>2, \\
{\left[\left(B^{(1)}-\left(v+\frac{1}{2}\right) x^{2}\right) \frac{1}{i k}\left(\partial_{x}-\frac{1-v}{x}\right)+B^{(2)} \frac{x^{2 v-1}}{i k}\left(\partial_{x}-\frac{v}{x}\right)\right] \psi(k x)=0,} \\
{\left[\left(B^{(0)}-x^{2 v+1}\right) \frac{1}{i k}\left(\partial_{x}-\frac{1-v}{x}\right)+\left(B^{(1)}-\left(v+\frac{1}{2}\right) x^{2}\right) \frac{x^{2 v-1}}{i k}\left(\partial_{x}-\frac{v}{x}\right)\right] \psi(k x)=0,} \\
\left(B^{(0)}-x^{2 v-1}\right) \frac{x^{2 v-1}}{i k}\left(\partial_{x}-\frac{v}{x}\right) \psi(k x)=0 .
\end{gathered}
$$

Observing the asymptotics for $x \rightarrow \infty$ in (7.4.11), we get that $B^{(0)}$ has order $2 v+1$. Then from (7.4.10) that $B^{(1)}$ has order 2 , from (7.4.9) that $B^{(2)}=0$ and finally from (7.4.8) by induction on $j$ that $B^{(j)}=0$ for $j>2$.

Recognizing $\frac{1}{i k}\left(\partial_{x}-\frac{1-v}{x}\right) \psi(k x)$ as the eigenfunction for the potential $(v-2)(v-1) / x^{2}$, obtained from $V_{0}$ by using $\phi_{0}(x)=x^{1-v}$, we see from (1.35) that necessarily

$$
B^{(1)}\left(k, \partial_{k}\right)=\left(v-\frac{1}{2}\right) \cdot\left(-\partial_{k}^{2}+\frac{(v-2)(v-1)}{k^{2}}\right) .
$$

On the other hand, $\frac{1}{i k}\left(\partial_{x}-\frac{v}{x}\right) \psi(k x)$ is the eigenfunction for the potential $v(v+1) / x^{2}$, obtained from $V_{0}$ by using $\phi_{0}(x)=x^{2}$. So if $v \in \frac{1}{2}+\mathbb{Z}_{>0}$, that is, we are in the even case, $2 v+1$ is even and we conclude:

$$
B^{(0)}\left(k, \partial_{k}\right)=\left(-\partial_{k}^{2}+\frac{v(v+1)}{k^{2}}\right)^{v+\frac{1}{2}} \text { if } v \in \frac{1}{2}+\mathbb{Z}_{>0} .
$$

If $v \in \mathbb{Z}_{>0}$, the rational $\mathrm{KdV}$ case, we get

$$
B^{(0)}\left(k, \partial_{k}\right)=i^{-(2 v+1)} \cdot\left(\partial_{k}-\frac{v}{k}\right) \circ \ldots \circ\left(\partial_{k}-\frac{1}{k}\right) \circ \partial_{k} \circ\left(\partial_{k}+\frac{1}{k}\right) \circ \ldots \circ\left(\partial_{k}+\frac{v}{k}\right) .
$$

For the proof, divide (7.4.11) by $x^{2 v+1} \cdot x^{2 v-1}$, and observing that, with $k x=y$, one has $\frac{1}{x} \partial_{k}=\partial_{y}=\frac{1}{k} \partial_{x}$, we have to prove that $\frac{1}{i}\left(\partial_{y}-\frac{v}{y}\right) \psi(y)$ is an eigenfunction, for the eigenvalue 1 , of the operator

$$
i^{-(2 v+1)}\left(\partial_{y}-\frac{v}{y}\right) \circ \ldots \circ\left(\partial_{y}-\frac{1}{y}\right) \circ \partial_{y} \circ\left(\partial_{y}+\frac{1}{y}\right) \circ \ldots \circ\left(\partial_{y}+\frac{v}{y}\right) .
$$


Now $\left(\partial_{y}+\frac{v}{y}\right)\left(\partial_{y}-\frac{v}{y}\right) \psi(y)=\left(\partial_{y}^{2}+\frac{v}{y^{2}}-\frac{v^{2}}{y^{2}}\right) \psi(y)=-\psi(y)$. Using that $\psi(y)$ $=\frac{1}{i}\left(\partial_{y}-\frac{v-1}{y}\right) \chi(y)$, where $\chi$ is the eigenfunction, for the eigenvalue 1 , of $-\partial_{y}^{2}+\frac{(v-2)(v-1)}{y^{2}}$, the result follows by induction on $v$.

Now Theorems 3.5 and 5.4 show that the $\Theta$ in (7.4.5) and $B=B^{(0)}+t \cdot B^{(1)}$ according to (7.4.12), (7.4.13), (7.4.14), satisfy $(B-\Theta) \phi_{\infty}^{+}=\phi_{\infty}^{+}$, so (7.4.10) has to hold. Conversely, one can give a direct proof of (7.4.10) by induction on $v$, which then yields an independent check on Theorem 3.5, respectively 5.4.

\subsection{Two Steps from Bessel: The Cusps}

Let $V$ be as in 7.4.3. Applying the recipe following (4.51), the generic element of $\operatorname{Ker}\left(-\partial_{x}^{2}+V\right)$, modulo a constant factor, is given by

$$
\tilde{\phi}_{0}(x)=\left(t \cdot x^{1-v}+x^{v}\right)^{-1} \cdot\left(\frac{t^{2}}{3-2 v} x^{3-2 v}+t x^{2}+\frac{1}{2 v+1} x^{2 v+1}+s\right),
$$

where $s$ is the integration constant. The potential obtained from $V$ by the Darboux transformation defined by $\tilde{\phi}_{0}$ is equal to

$$
\tilde{V}(x)=\frac{(v-3)(v-2)}{x^{2}}-2(\log \xi(x))^{\prime \prime}
$$

with

$$
\xi(x)=x^{4 v-2}+(2 v+1) \cdot t \cdot x^{2 v-1}+(2 v-1) \cdot s \cdot x^{2 v-3}+\frac{2 v+1}{3-2 v} t^{2}
$$

We recognize all the families in 7.1, 7.2, with at least two parameters, all of which except the last two are equal to zero, as being of this form.

Solving $\xi(p)=0, \xi^{\prime}(p)=0$, we find that $\xi$ has a multiple zero at $x$ if and only if

$$
t=-\varepsilon^{2 v-1}, \quad s=\frac{(2 v-1)^{2}}{(2 v-3) \cdot(2 v+1)} \varepsilon^{2 v+1}
$$

and

$$
\begin{array}{ll}
p=\varepsilon \quad \text { if } \quad v \in \mathbb{Z}_{>0} \quad(\mathrm{KdV} \text { case }) \\
& \text { respectively } p= \pm \varepsilon \text { if } v \in \frac{1}{2}+\mathbb{Z}_{>0} \quad \text { (even case) } .
\end{array}
$$

These multiple zeros $p$ can only be triple zeros because they are obtained from applying a Darboux transformation with a $\tilde{\phi}_{0}$ which is recessive at a pole $p \neq 0$ of $V$. There we had $v_{p}=1$, so $\tilde{v}_{p} \leqq 2$. It follows that for the minimal order $m$ of the differential equation in $k$ we get, if $t \neq 0$,

$$
\begin{aligned}
& \tilde{m} \leqq 4 v-1, \quad \text { respectively } \tilde{m} \leqq 4 v \text {, off the cusp (7.5.4), } \\
& \text { if } v \in \mathbb{Z}_{>0}, \text { respectively } v \in \frac{1}{2}+\mathbb{Z}_{>0},
\end{aligned}
$$

$\tilde{m} \leqq 4 v-2$ on the cusp (7.5.4), both in the $\mathrm{KdV}$ case and in the even case. 
We do not have a general formula for the $\Theta$ and $B$ of minimal order either on or off the cusps.

But in the cases $v=2, v=2 \frac{1}{2}$ we give these minimal $\Theta$ and $B$ below. The case $v=2$ corresponds to $\theta_{3}$ in 7.1 with the parameters $p_{3}, p_{5}$. The cusp condition reads in these parameters: $p_{3}^{5}=p_{5}^{3}$. Writing $p_{3}=s^{3}, p_{5}=s^{5}$, the triple pole is situated at $x=s$. The corresponding $\Theta$ and $B\left(k, \partial_{k}\right)$ are:

$$
\begin{gathered}
\Theta(x)=x^{6}-\frac{3}{2} s^{2} x^{4}-14 s^{3} x^{3}-9 s^{4} x^{2}+60 s^{5} x \\
B=\left(-\partial_{k}^{2}+\frac{12}{k^{2}}\right)^{3}-s^{2}\left[\partial_{k}^{4}-\frac{24}{k^{2}} \partial_{k}^{2}+\frac{48}{k^{3}} \partial_{k}+\frac{72}{k^{4}}\right] \\
-14 i s^{3}\left[\partial_{k}^{3}-\frac{81}{7 k^{2}} \partial_{k}+\frac{81}{7 k^{3}}\right]-9 s^{4}\left(-\partial_{k}^{2}+\frac{12}{k^{2}}\right)-60 i s^{5} \partial_{k} .
\end{gathered}
$$

The case $v=\frac{1}{2}+2$ corresponds to $V_{4}$ in 7.2 with the parameters $t_{2}, t_{3}$. The cusp appears at $\overline{t_{2}=-3 \cdot t^{2}}, t_{3}=30 \cdot t^{3}$. Then

$$
V_{4}(x)=-\frac{1}{4 x^{2}}+\frac{2}{(x+i \sqrt{6 \cdot t})^{2}}+\frac{2}{(x-i \sqrt{6 \cdot t})^{2}}+\frac{6}{(x+\sqrt{2 \cdot t})^{2}}+\frac{6}{(x-\sqrt{2 \cdot t})^{2}} .
$$

The $\Theta$ and $B$ of minimal order are:

$$
\begin{aligned}
\Theta=x^{8}-56 t^{2} x^{4}+192 t^{3} x^{2} \\
B=\left(-\partial_{k}^{2}+\frac{63}{4 k^{2}}\right)^{4}-56 t^{2}\left(-\partial_{k}^{4}-\frac{249}{14 k^{2}} \partial_{k}^{2}+\frac{249}{7 k^{3}} \partial_{k}+\frac{2385}{112 k^{4}}\right) \\
+192 t^{3}\left(-\partial_{k}^{2}-\frac{1}{4 k^{2}}\right) .
\end{aligned}
$$

Because of their different behavior, the cusps should be distinguished from the vertices with $\mu=2$ in Diagram 4-1 in the even case. Adding the cusps leads to a refined diagram as below. For clarity we only have drawn the potentials obtained from Bessel by at most two rational Darboux transformations, and we have deleted the dashed arrows, keeping, however, the arrows which indicate approximation.

A similar diagram can be drawn in the rational $\mathrm{KdV}$ case. In 7.7 the situation is described for the potentials obtained in $\leqq 4$ steps from $V=0$.

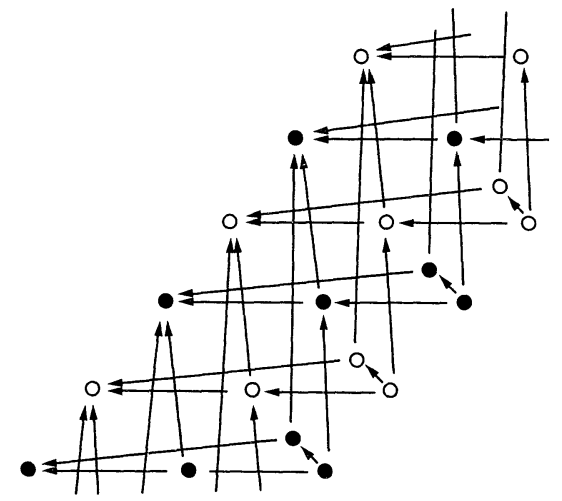




\subsection{The $K d V$ Case with Parameters $p_{3}, p_{5}, p_{7}$}

In this section we return to the potential $V(x)$ arising from

$$
\theta_{4}(x)=x^{10}-5 p_{3} x^{7}+63 p_{5} x^{5}-225 p_{7} x^{3}+315 p_{3} p_{5} x^{2}-175 p_{3}^{3} x+225 p_{3} p_{7}-189 p_{5}^{2} \text {. }
$$

In 7.1 we have given, for any value of the parameters $\left(p_{3}, p_{5}, p_{7}\right)$ an explicit formula for an operator $B\left(k, \partial_{k}\right)$ of order eleven such that $B \phi=\Theta \phi$. We now investigate for which $p_{3}, p_{5}, p_{7}$ one can find an operator of lower order.

Most of the computations up to (7.6.14) were carried out using Vaxima. Most of those from (7.6.15) on were done directly by hand.

We use the eigenfunction $\phi^{+}(x, k)$ [see (7.1.2)] and we consider the equation

$$
k^{-4}\left(\sum_{p=0}^{11} \tilde{B}_{p}(k) \partial_{k}^{p}\right) k^{4} \phi^{+}(x, k)=\left(\sum_{r=0}^{11} \Theta_{r} x^{r}\right) \phi^{+}(x, k)
$$

for the unknowns $\Theta_{r}$ and $\tilde{B}_{p}(k)$.

The advantage in conjugating $B\left(k, \partial_{k}\right)$ by the function $k^{4}$ is that (7.6.1) is equivalent to a finite number of conditions: if one multiplies the difference between both sides of (7.6.1) by $e^{-i k x}$ one obtains a polynomial in $x$ of degree 21 . The vanishing of the coefficients of the powers running down from 21 to 10 determine the operator $B\left(k, \partial_{k}\right)$ and the vanishing of the remaining ten coefficients imposes relations among the $\Theta_{r}$. For example, from the coefficient of $x^{9}$ one gets the relations

$$
\begin{aligned}
\Theta_{7}= & -20 \Theta_{10} p_{3}, \\
\Theta_{5}= & 138 \Theta_{10} p_{5}-660 \Theta_{11} p_{3}^{2}-32 \Theta_{8} p_{3}, \\
\Theta_{3}= & -700 \Theta_{10} p_{7}+\left(9240 \Theta_{11} p_{3}+224 \Theta_{8}\right) p_{5}-780 \Theta_{9} p_{3}^{2}-30 \Theta_{6} p_{3}, \\
\Theta_{1}= & {\left[-\left(\left(69300 \Theta_{11} p_{3}+2520 \Theta_{8}\right) p_{7}+47817 \Theta_{11} p_{5}^{2}\right.\right.} \\
& \left.\left.+\left(-18144 \Theta_{9} p_{3}-378 \Theta_{6}\right) p_{5}+4500 \Theta_{10} p_{3}^{3}+36 \Theta_{4} p_{3}\right) / 2\right] .
\end{aligned}
$$

The analysis of the remaining relations (those from $x^{s}, 0 \leqq s \leqq 7$, since $x^{8}$ gives redundant information) among the $\Theta_{r}$ depends on the possible vanishing of $p_{3}$.

We first dispose of the case

$$
p_{3}=0 \text { ("two steps from Bessel"). }
$$

Assume first that $p_{5} \neq 0$. Then one can solve all the "remaining relations" to obtain

$$
\begin{aligned}
& \Theta_{9}=\frac{-20 \Theta_{10} p_{7}^{3}}{9 p_{5}^{4}}, \\
& \Theta_{8}=\frac{5 \Theta_{10} p_{7}}{4 p_{5}}, \\
& \Theta_{6}=\frac{231 \Theta_{11} p_{5}^{3}-10 \Theta_{10} p_{7}^{2}}{2 p_{5}^{2}}, \\
& \Theta_{4}=\frac{621 \Theta_{9} p_{5}^{4}-2475 \Theta_{11} p_{5}^{3} p_{7}-150 \Theta_{10} p_{7}^{3}}{4 p_{5}^{3}}, \\
& \Theta_{2}=\frac{1575 \Theta_{10} p_{7}^{4}}{p_{5}^{4}},
\end{aligned}
$$

$\Theta_{10}\left(3 p_{7}^{5}-p_{5}^{7}\right)=0$ 
$\Theta_{10}=\Theta_{11}=0$ implies $\Theta_{r}=0$ for $r \geqq 1$, that is, $\Theta$ is a constant. So the order of the operator is at least 10 . For order 10 we get [see $\left.\left(7.6 .3^{\prime}\right)\right]$ the necessary condition

$$
3 p_{7}^{5}-p_{5}^{7}=0 \quad \text { (the "cusp in two steps from Bessel"). }
$$

If we take $p_{5}=0$, i.e. consider

$$
p_{3}=p_{5}=0, \quad p_{7} \neq 0 \quad \text { (“one step from Bessel"), }
$$

then the "remaining relations" give

$$
\Theta(x)=\Theta_{11}\left(x^{11}-\frac{2475}{4} p_{7} x^{4}\right)+\Theta_{9}\left(x^{9}-\frac{2025}{9} p_{7} x^{2}\right),
$$

and the order is at least 9. An operator of order 9 is given in Sect. 7.4.

From now on assume that $p_{3} \neq 0$.

From the coefficient of $x^{7}$ one gets to solve for $\Theta_{6}, \Theta_{4}, \Theta_{2}$ in terms of the $\Theta_{r}$, $8 \leqq r \leqq 11$. Combining with (7.6.2) we get, for example,

$$
\begin{aligned}
& \Theta_{7}=-20 \Theta_{10} p_{3}, \\
& \Theta_{6}=33 \Theta_{11} p_{5}+5 \Theta_{10} \frac{p_{7}}{p_{3}}-21 \Theta_{9} p_{3}-4 \Theta_{8} \frac{p_{5}}{p_{3}}, \\
& \Theta_{5}=-660 \Theta_{11} p_{3}^{2}+138 \Theta_{10} p_{5}-32 \Theta_{8} p_{3} .
\end{aligned}
$$

The remaining expressions are too long to report here. Since they all give $\Theta_{r}$, $1 \leqq r \leqq 7$ as linear combinations of $\Theta_{r}, 8 \leqq r \leqq 11$, we conclude that the degree of a nonconstant $\Theta$ is at least 8. Under the assumption of order 8 , that is, $\Theta_{9}=\Theta_{10}$ $=\Theta_{11}=0$, the remaining relations (those from $x^{s}, 0 \leqq s \leqq 5$, since $x^{6}$ gives redundant information) simplify considerably and turn out to force

$$
p_{j}=t^{j}, \quad j=3,5,7, \text { for some } t \in \mathbb{C} .
$$

Conversely, if (7.6.6) holds then all equations can be solved and an operator of order 8 exists. This confirms Theorem 3.5 neatly: in this case $\theta$ has a 6 -fold zero at $x=t: v_{t}=3$, and the remaining zeros are simple. So (3.34) represents $3+4=7$ linear relations for $\Theta^{\prime}$, so Theorem 3.5 predicts the existence of a non-constant $\Theta$ of degree 8. When (7.6.6) holds one can also produce operators $B\left(k, \partial_{k}\right)$ of order higher than eight. We ignore the case (7.6.6) from now on.

We proceed to identify the cases when we have operators of order ten and nine, with $p_{3} \neq 0$. Set $\Theta_{11}=0$ in the "remaining relations" (from $x^{s}, 0 \leqq s \leqq 5$ ) and consider first the case

$$
p_{3}^{5}=p_{5}^{3} \quad\left(p_{j}=t^{j}, j=3,5\right) .
$$

This gives $\Theta_{8}, \Theta_{9}$ in terms of $\Theta_{10}$ and shows that if $\Theta$ is nonconstant the order is 10 . Moreover, we are forced to choose

$$
p_{7}=\beta t^{7}
$$

with $\beta$ a solution of

$$
25 \beta^{3}+54 \beta^{2}+192 \beta+104=0 .
$$

This equation has one real root $(\cong-0.618)$. 
When $p_{3}^{5} \neq p_{5}^{3}$ one can use the first of the "remaining equations" to solve for $\Theta_{8}$, and then one sees that all the "remaining equations" reduce to a pair of linear homogeneous equations for $\Theta_{9}, \Theta_{10}$, namely $m_{11} \Theta_{10}+m_{12} \Theta_{9}=0, m_{21} \Theta_{10}$ $+m_{22} \Theta_{9}=0$, with

$$
\begin{aligned}
m_{11}= & 10\left(5 p_{3} p_{7}^{4}-14 p_{5}^{2} p_{7}^{3}-15 p_{3}^{4} p_{5} p_{7}^{2}+53 p_{3}^{3} p_{5}^{3} p_{7}-p_{3}^{8} p_{7}-20 p_{3}^{2} p_{5}^{5}-8 p_{3}^{7} p_{5}^{2}\right), \\
m_{12}= & 9\left(10 p_{3}^{3} p_{7}^{3}-48 p_{3}^{2} p_{5}^{2} p_{7}^{2}+48 p_{3} p_{5}^{4} p_{7}+18 p_{3}^{6} p_{5} p_{7}-7 p_{5}^{6}-24 p_{3}^{5} p_{5}^{3}+3 p_{3}^{10}\right), \\
m_{21}= & 10\left(14 p_{5} p_{7}^{4}+10 p_{3}^{4} p_{7}^{3}-42 p_{3}^{3} p_{5}^{2} p_{7}^{2}+2 p_{3}^{2} p_{5}^{4} p_{7}\right. \\
& \left.-4 p_{3}^{7} p_{5} p_{7}+3 p_{3} p_{5}^{6}+16 p_{3}^{6} p_{5}^{3} p_{3}^{11}\right), \\
m_{22}= & 9\left(28 p_{3}^{2} p_{5} p_{7}^{3}-41 p_{3} p_{5}^{3} p_{7}^{2}+5 p_{3}^{6} p_{7}^{2}+7 p_{5}^{5} p_{7}-19 p_{3}^{5} p_{5}^{2} p_{7}+26 p_{3}^{4} p_{5}^{4}-6 p_{3}^{9} p_{5}\right) .
\end{aligned}
$$

For a non-trivial solution of this pair of equations we need

$$
\begin{aligned}
\Delta \equiv & \left(25 p_{3} p_{7}^{6}-21 p_{5}^{2} p_{7}^{5}+105 p_{3}^{4} p_{5} p_{7}^{4}-350 p_{3}^{3} p_{5}^{3} p_{7}^{3}+15 p_{3}^{8} p_{7}^{3}\right. \\
& +315 p_{3}^{2} p_{5}^{5} p_{7}^{2}-105 p_{3}^{7} p_{5}^{2} p_{7}^{2}-90 p_{3} p_{5}^{7} p_{7}+210 p_{3}^{6} p_{5}^{4} p_{7} \\
& \left.+7 p_{5}^{9}-105 p_{3}^{5} p_{5}^{6}-7 p_{3}^{10} p_{5}^{3}+p_{3}^{15}\right)=0 .
\end{aligned}
$$

Notice that for $p_{3}=0$ we get $\Delta=7 p_{5}^{2}\left(p_{5}^{7}-3 p_{7}^{5}\right)$, and that for $p_{3}=t^{3}, p_{5}=t^{5}, p_{7}=\beta t^{7}$, we get that $\Delta=0$ is equivalent to $\beta=1$ or (7.6.9). It is also clear that a solution with $\Theta_{10}=0$ requires

$$
m_{12}=m_{22}=0 \text {. }
$$

At this moment we use the weighted homogeneity of $\theta$ in $x=p_{1}, p_{3}, p_{5}, p_{7}$ to see that the equations for $\Theta$ are equivalent along each complex curve

$$
s \mapsto\left(p_{3} s^{3}, p_{5} s^{5}, p_{7} s^{7}\right), \quad s \in \mathbb{C} \backslash\{0\} .
$$

(This corresponds to the homothety $x \mapsto x \cdot s$ and therefore is equivalent to the algebras of $\Theta$ 's being isomorphic. See the remarks following Proposition 3.6.)

Because we have already disposed of the case $p_{3}=0$, we may therefore take $p_{3}=1$. Then the resultant of Eqs. (7.6.11) for $p_{7}$ is given by

$$
\left(p_{5}^{3}-1\right)^{4}\left(p_{5}^{2}+p_{5}+1\right)\left(16807 p_{5}^{9}+2855193 p_{5}^{6}+166233 p_{5}^{3}-625\right)=0,
$$

and for each solution $p_{5}$ of (7.6.13) there is a unique solution $p_{7}$ of (7.6.11) (with $\left.p_{3}=1\right)$.

Multiplying $p_{5}$ with $\omega$ such that $\omega^{3}=1$ leads to another solution on the same curve (7.6.12). Equation (7.6.13) turns out to have four real roots for $p_{5}^{3}$ and $p_{5}^{3}=1$ corresponds to (7.6.6). So apart from $p_{3}=p_{5}=0, p_{7} \neq 0$ and (7.6.6), there are three more curves (7.6.12) along which there is an operator of order 9 , and each of these contains a point with $p_{3}=1$ and $p_{5}, p_{7}$ real.

The real solutions of (7.6.8), with $p_{3}=1$ and $p_{5}^{3} \neq 1$ are given by the pairs

$$
\begin{aligned}
& \left(p_{5}, p_{7}\right) \cong(-0.395,0.777), \\
& \left(p_{5}, p_{7}\right) \cong(-5.538,5.265), \\
& \left(p_{5}, p_{7}\right) \cong(0.152,-0.500) .
\end{aligned}
$$

Summary 1. $B\left(k, \partial_{k}\right)$ of order 10 requires (7.6.10). For order 9 one needs (7.6.11) (this includes $p_{3}=p_{5}=0$ ) and (7.6.6) is necessary for order 8 . 
We now turn to an interpretation of Eqs. (7.6.10), (7.6.11) in terms of the geometry of the roots of $\theta_{4}$. The first point is that the equation $\Delta=0$ is also the resultant of $\theta_{4}(x)=0$ and $\theta_{4}^{\prime}(x)=0$, as can be checked directly. It can also be concluded from Theorem 3.5: if $\theta_{4}$ has ten simple zeros $p\left(v_{p}=1\right.$ for all $p$ ) then $\Theta^{\prime}$, having the same zeros, is of degree $\geqq 10, \operatorname{deg} \Theta \geqq 11$. Conversely, multiple zeros of $\theta_{4}$ lead to the existence of $\Theta$ of lower degree, as observed at the end of Sect. 3.

Any multiple zero of $\theta_{4}$ has at least multiplicity three. This fact, discovered for all $\theta$ 's by Airault-McKean-Moser [3], see also (3.33), may be checked directly by showing that the resultant of $\theta_{4}$ and $\theta_{4}^{\prime}$ divides the resultant of $\theta_{4}$ and $\theta_{4}^{\prime \prime}$. Now let $\theta_{4}$ have a triple zero at $t$.

It is easy to see that $t=0$ if and only if $p_{3}=p_{5}=0$. This is the case of an operator $B$ of order 9 , characterized geometrically by the fact that the poles of $V$ form a regular heptagon around the origin, where we have a pole with coefficient $=6$. But there is more: this is the only case where the remaining seven zeros of $\theta_{4}$ are simple and we nevertheless have an operator of order 9 . For the proof, divide $\theta_{4}$ by $(x-t)^{3}$ :

$$
\theta_{4}(x)=(x-t)^{3} \cdot Q_{t}(x)
$$

where we assume that $Q_{t}$ has seven simple zeros. If $\operatorname{deg} \Theta=9$, then $\Theta^{\prime}(x)$ must be a constant multiple of $(x-t) \cdot Q_{t}(x)$ and the condition $\Theta^{\prime \prime \prime}(t)=0$ [cf. (3.34)] is equivalent to $\left.\frac{\partial}{\partial x} Q_{t}(x)\right|_{x=t=0}$. Combining this equation with $\theta_{4}^{\prime \prime}(t)=0, \theta_{4}^{\prime}(t)=0$ one readily obtains $t=0$ or (7.6.6), where the last case is ruled out by the condition that $Q_{t}$ has only simple zeros.

Assuming from now on that $t=0, p_{3} \neq 0$, we may restrict to $\underline{p_{3}}=1$, and then the equations $\theta_{4}^{\prime \prime}(t)=0, \theta_{4}^{\prime}(t)=0$ can be combined to give the following parametrization of $\Delta=0$ :

$$
\begin{aligned}
& p_{5}(t)=\frac{1}{9}\left(-t^{5}+5 t^{2}+\frac{5}{t}\right), \\
& p_{7}(t)=\frac{1}{27}\left(-t^{7}+21 t+\frac{7}{t^{2}}\right),
\end{aligned}
$$

Writing $\gamma(t)=\left(p_{5}(t), p_{7}(t)\right)$, we get $\gamma^{\prime}(t) \neq 0$ unless $t=1$, which is the point of $\Delta=0$ corresponding to (7.6.6), for which $\theta_{4}$ had a sixfold zero. At this point the hypersurface $\Delta=0$ (restricted to the transversal section $p_{3}=1$ ) has a singularity which is locally diffeomorphic to the cusp $\xi^{3}=\eta^{5}$, a singularity of type $E_{8}$ in Arnol'd's classification.

We get that $\Delta=0$ is smooth at $p_{5}, p_{7}$ if there is only one $t \in \mathbb{C} \backslash\{0\}$ such that $\gamma(t)$ $=\left(p_{5}, p_{7}\right)$. Now each $t$ such that $\gamma(t)=\left(p_{5}, p_{7}\right)$ is a triple zero of $\theta_{4}$ with coefficients $p_{3}=1, p_{5}, p_{7}$. Three of such $t$ would, in view of Theorem 3.5, imply the existence of a $\Theta$ of degree 8, which we know can only occur if (7.6.6) holds - which is another configuration of one sixfold zero and four simple ones. Two such $t$ imply the existence of a $\Theta$ of degree 9, which brings us to the case (7.6.11). We know that there are three curves of the form (7.6.12), each of which intersects $p_{3}=1, p_{5}$ and $p_{7}$ real. Now $p_{5}(t) \in \mathbb{R}, p_{7}(t) \in \mathbb{R}$ can occur in two ways: either $t \in \mathbb{R}$ or,

$$
p_{5}(t)=p_{5}(\bar{t}), \quad p_{7}(t)=p_{7}(\bar{t}), \quad t \neq \bar{t} .
$$


In the last case we have a complex conjugate pair of triple zeros for $\theta_{4}$. This turns out to occur in two cases:

$$
t \cong-0.965 \pm 1.479 \sqrt{-1}, \text { respectively } t \cong 0.532 \pm 1.951 \sqrt{-1}
$$

corresponding to the isolated points

$$
p_{5} \cong-0.393, \quad p_{7} \cong 0.777, \text { respectively } p_{5} \cong-5.538, \quad p_{7} \cong 5.265
$$

in the locus $\Delta=0, p_{3}=1, p_{5}$ and $p_{7}$ real.

On the other hand, there is one self-intersection of the curve $\gamma(t), t \in \mathbb{R} \backslash\{0\}$, the images for

$$
t=t_{1} \cong-1.019 \text { and } t=t_{2} \cong 1.762
$$

are equal to

$$
\left(p_{5}, p_{7}\right) \cong(0.15,-0.50) .
$$

At all these points $\Delta=0$ has an ordinary double point in the complex domain: it is locally equal to the union of two smooth hypersurfaces with transversal tangent spaces.

The locus $\Delta=0$ in $p_{3}=1, p_{5}$ and $p_{7}$ real is sketched in Diagram 7.6-1 below.

Since (7.6.11), except from the case (7.6.6), has only three cases, these must coincide with the double points described above. This is neatly checked by the numerical values reported in (7.6.14), (7.6.19), (7.6.21). Noting also that $\Delta=0$ is smooth at $p_{3}=p_{5}=0, p_{7} \neq 0$, we get a further

Summary 2. $B\left(k, \partial_{k}\right)$ of order $\leqq 10$ is equivalent to $\theta_{4}$ having multiple zeros. Minimal order 9 occurs at (i) $p_{3}=p_{5}=0, p_{7} \neq 0$, where $\theta_{4}$ has one triple zero and seven simple zeros and the discriminant locus $\Delta=0$ of $\theta_{4}$ is smooth; (ii) three curves (7.6.12), where $\theta_{4}$ has two triple zeros and four simple zeros and along which $\Delta=0$ has ordinary double points. Minimal order 8 occurs at $p_{3}=t^{3}, p_{5}=t^{5}, p_{7}=t^{7}$ $(t \neq 0)$, where $\theta_{4}$ has a sixfold zero and four simple zeros, and $\Delta=0$ has a singularity of type $E_{8}$ in a transversal section. At all other points of $\Delta=0$ the minimal order is equal to 10 ; there is one triple zero and seven simple ones and $\Delta=0$ is smooth.

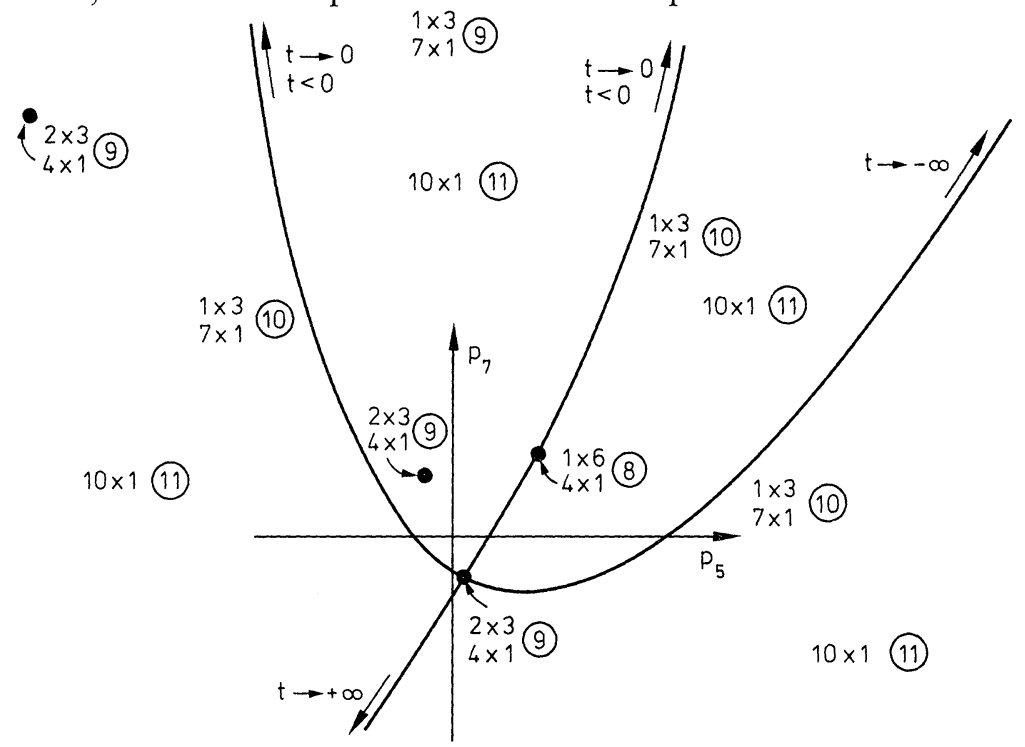

Diagram 7.6-1. $\Delta=0$ for $p_{3}=1, p_{5}$ and $p_{7}$ real 
Remarks. i) $p_{3}=p_{5}=0, p_{7} \neq 0$ is the exceptional case where a decrease in the minimal order of $B$ does not correspond to a change in the multiplicities of the zeros of $\theta_{4}$, or to a singularity in the discriminant locus of $\theta_{4}$.

ii) $(\Delta=0) \backslash\left(p_{3}=p_{5}=0\right)$ can also be parametrized by $p_{j}=\lambda_{1}^{j}+\lambda_{2}^{j}, j=3,5,7$ and $\lambda_{1}, \lambda_{2} \in \mathbb{C}$. This is inspired by the interpretation in 7.1 of the $p_{j}$ as the power sums of eigenvalues of matrices. However, the equations, for instance for the double points, do not look more manageable in these coordinates.

iii) If one wants to follow which configurations are met by a real potential during the $\mathrm{KdV}$-flow ( $p_{3}$ running, $p_{5}$ and $p_{7}$ fixed) then, in view of (7.6.12), one has to follow the curves $\left.t \hookrightarrow p_{5} \cdot t^{-5}, p_{7} \cdot t^{-7}\right)$ in Diagram 7.6-1 and see where they meet $\Delta=0$.

\subsection{Limiting Relations Among the KdV Potentials}

If, in the definition of the $\theta_{v}$ via (7.1.1) we write

$$
p_{i}=q_{i}+t^{i}, \quad q_{i}=\sum_{j=1}^{v-1}\left(\varepsilon_{j}\right)^{i}, \quad t=\varepsilon_{v},
$$

then we see immediately that

$$
\begin{aligned}
\operatorname{det}\left(\left(\varepsilon_{j}\right)^{2 i-1}\right)_{1 \leqq i, j \leqq \nu}= & t^{2 v-1} \cdot\left(\operatorname{det}\left(\left(\varepsilon_{j}\right)^{2 i-1}\right)_{i, j \leqq \nu-1}\right. \\
& + \text { lower order in } t, \\
\operatorname{det}\left(\left(\varepsilon_{j}\right)^{i-1}\right)_{1 \leqq i, j \leqq \nu-1}= & t^{\nu-1}\left(\operatorname{det}\left(\left(\varepsilon_{j}\right)^{2 i-1}\right)_{i, j \leqq \nu-1}\right. \\
& + \text { lower order in } t .
\end{aligned}
$$

Therefore

$$
\lim _{t \rightarrow \infty} t^{-v} \cdot \theta_{v}\left(x+t, p_{3}+t^{3}, \ldots, p_{2 v-1}+t^{2 v-1}\right)=\theta_{v-1}\left(x, q_{3}, \ldots, q_{2 v-3}\right),
$$

showing in an explicit way that each potential of order $v-1$ can be approximated by potentials of order $v$, as expressed in Diagram 3-1.

Let us now consider in more detail the limiting relations between the various configurations which are possible for potentials of order $\leqq 4$. We know that the corresponding $\theta$ has, in general position, one, three, six, or ten simple zeros. We denote such configurations by $1 \times 1,3 \times 1,6 \times 1,10 \times 1$. When we have two parameters we can also have $\left(p_{3}^{5}=p_{5}^{3}\right)$ one triple zero and three simple zeros: we denote this by

$$
\begin{aligned}
& 1 \times 3 \\
& 3 \times 1
\end{aligned} .
$$

Finally, for the case of three parameters (see 7.6), we can have the configurations denoted by

$$
\begin{array}{ll}
1 \times 3 & 1 \times 6 \\
7 \times 1 & 4 \times 1
\end{array}, \text { and } \begin{aligned}
& 2 \times 3 \\
& 4 \times 1
\end{aligned} .
$$

Using arrows to denote the approximation of potentials by motion of the parameters as in Sect. 3, we get the following refinement of Diagram 3-1. The 
numbers in the circles denote the minimal positive degree of $B\left(k, \partial_{k}\right)$ and $\Theta$. Like in Diagram 4-1 the diagonal elements are the Bessel potentials.

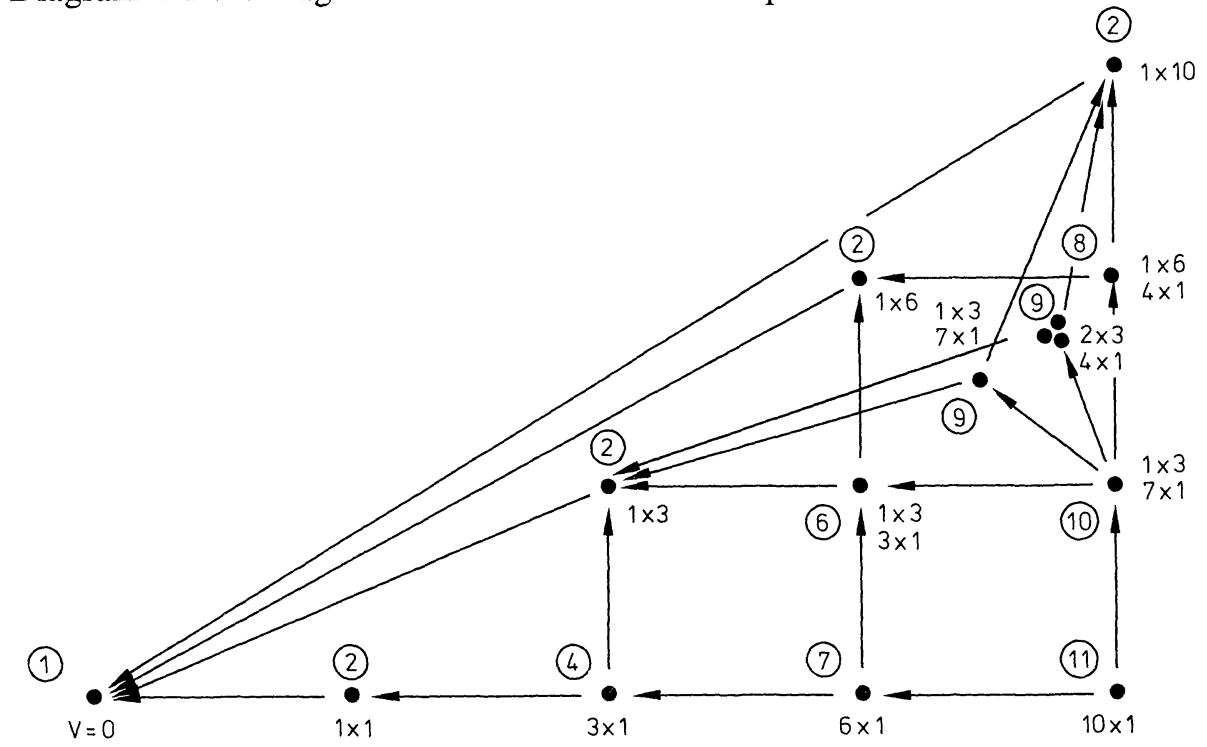

Diagram 7.8-1

\section{References}

1. Ablowitz, M., Airault, H.: Perturbations finies et forme particulaire de certaines solutions de l'équation de Korteweg-deVries. C.R. Acad. Sci. Paris 292, Serie I, 279-281 (1981)

2. Adler, M., Moser, J.: On a class of polynomials connected with the Korteweg-deVries equations. Commun. Math. Phys. 61, 1-30 (1978)

3. Airault, H., McKean, H.P., Moser, J.: Rational and elliptic solutions of the Korteweg-deVries equation and a related many body problem. Commun. Pure Appl. Math. 30, 95-148 (1977)

4. Bargman, V.: On the connection between phase shifts and scattering potential. Rev. Mod. Phys. 21, 489-493 (1949)

5. Burchnall, J.L., Chaundy, T.W.: Commutative ordinary differential operators. Proc. Lond. Math. Soc. 21, 420-440 (1923)

6. Darboux, G.: Leçons sur la théorie générale des surfaces, 2ème partie. Paris: Gauthiers-Villars 1889

7. Date, E., Jimbo, M., Kashiwara, M., Miwa, T.: Operator approach to the KadomtsevPetviashvili equation. Transformation groups for soliton equations. III. J. Phys. Soc. Jpn. 50, 3806-3812 (1981)

8. Flaschka, H., Newell, A.: Monodromy and spectrum preserving deformations. Commun. Math. Phys. 76, 65-116 (1980)

9. Gelfand, I.M., Dikii, L.: Fractional powers of operators and Hamiltonian systems. Funkts. Anal. Prilozh. 10, No. 4, 13-39 (1976)

10. Giertz, M., Kwong, M.K., Zettl, A.: Commuting linear differential expressions. Proc. R. Soc. Edinb. 87 A, 331-347 (1981)

11. Grünbaum, F.A.: The limited angle reconstruction problem in computed tomography. Proc. Symp. Appl. Math., Vol. 27, AMS, L. Shepp (ed.), pp. 43-61 (1982)

12. Grünbaum, F.A.: A new property of reproducing kernels for classical orthogonal polynomials. J. Math. Anal. Appl. 95, 491-500 (1983)

13. Grünbaum, F.A.: Band and time limiting, recursion relations, and some nonlinear evolution equations. In: Special functions: group theoretical aspects and applications. Askey, R., Koorwinder, T., Schempp, W. (eds.), pp. 271-286. Dordrecht: Reidel 1984 
14. Hartshorne, R.: Algebraic geometry. Berlin, Heidelberg, New York: Springer 1977

15. Hurwitz, A.: Über die Bedingungen, unter welchen eine Gleichung nur Wurzeln mit negativen realen Teilen besitzt. Math. Ann. 46, 273-284 (1895)

16. Kac, V.: Infinite dimensional Lie algebras. Boston: Birkhäuser 1983

17. Krichever, I.M.: Methods of algebraic geometry in the theory of nonlinear equations. Usp. Mat. Nauk. 32, 183-208 (1977); Russ. Math. Surv. 32, 185-213 (1977)

18. Mehta, M.: Random matrices. New York: Academic Press 1967

19. Olver, F.W.J.: Asymptotics and special functions. New York: Academic Press 1974

20. Poincaré, H.: Sur les intégrales irrégulières des équations linéaires. Acta Math. 8, 295-344 (1886)

21. Sato, M.: Soliton equations as dynamical systems on an infinite dimensional Grassmann manifold. RIMS Kokyuroku 439, 30-46 (1981)

22. Schur, I.: Über vertauschbare lineare Differential-Ausdrücke. Sitzungsber. Berl. Math. Ges. 4, 2-8 (1905). See also Ges. Abhandlungen, I, pp. 170-176. Berlin, Heidelberg, New York: 1973

23. Segal, G., Wilson, G.: Loop groups and equations of KdV type. Publicat. Math. IHES 1985

24. Slepian, D.: Some comments on Fourier analysis, uncertainty, and modeling. SIAM Rev. 25, 379-394 (1983)

25. Szegö, G.: Über eine Eigenschaft der Exponentialreihe. Sitzungsber. Akad. Berl. Math. Ges. 4, 2-8 (1905). See also Ges. Abhandlungen, I, pp. 170-176. Berlin, Heidelberg, New York: Springer 1973

Communicated by L. Nirenberg

Received December 17, 1984; in revised form July 16, 1985 